\title{
THE SPLITTING LEMMAS FOR NONSMOOTH FUNCTIONALS ON HILBERT SPACES II. THE CASE AT INFINITY
}

\author{
GuAngCun LU
}

\begin{abstract}
We generalize the Bartsch-Li's splitting lemma at infinity for $C^{2}$-functionals in [2] and some later variants of it to a class of continuously directional differentiable functionals on Hilbert spaces. Different from the previous flow methods our proof is to combine the ideas of the MorsePalais lemma due to Duc-Hung-Khai [9] with some techniques from [11], [17], [18]. A simple application is also presented.
\end{abstract}

\section{Introduction and main results}

The Gromoll-Meyer's generalized Morse lemma (so called splitting lemma) is one of key results in infinite dimensional Morse theory. As a supplement of it, Thomas Bartsch and Shujie Li proved in 1997 a splitting lemma at infinity (see [2]) and used it to develop a kind of Morse theory to study some variational problem without compactness ([2], [10] and [12]). Recently, Shaowei Chen and Shujie Li generalized it [5] (in a Hilbert space frame) and [6] (in a Banach space frame). These were successfully used by them in studying problems with (strong) resonance. Their proof adopted the flow method as done for the usual splitting lemma as in [4], [16]. So the functionals are assumed to be at least $C^{2}$. Based on

2010 Mathematics Subject Classification. Primary 58E05, 49J52, 49J45.

Key words and phrases. Nonsmooth functional, splitting lemma at infinity, elliptic boundary value problems.

Partially supported by the NNSF 10971014 and 11271044 of China, and PCSIRT and Research Fund for the Doctoral Program Higher Education of China (Grant No. 200800270003). 
the proof ideas of the Morse-Palais lemma due to Duc-Hung-Khai [9] and some techniques from [11], [17], [18] we find a new method to establish the splitting theorems for nonsmooth functionals on Hilbert spaces in [13], [14]. We shall follow the notations therein.

Recall that a neighbourhood of infinity in a Banach space $\left(X,\|\cdot\|_{X}\right)$ is a set containing $\left\{u \in X \mid\|u\|_{X}>R\right\}$ for some $R>0$. A map $A$ from a neighbourhood of infinity in $X$ to $X$ is said to be strictly Fréchet differentiable at $\infty$ if there exists an operator $A^{\prime}(\infty) \in \mathcal{L}(X)$ such that

$$
\frac{\left\|A\left(x_{1}\right)-A\left(x_{2}\right)-A^{\prime}(\infty)\left(x_{1}-x_{2}\right)\right\|_{X}}{\left\|x_{1}-x_{2}\right\|_{X}} \rightarrow 0,
$$

as $x_{1} \neq x_{2}$ and $\left(\left\|x_{1}\right\|_{X},\left\|x_{2}\right\|_{X}\right) \rightarrow(\infty, \infty)$. (We also say that $A$ has a strict Fréchet derivative $A^{\prime}(\infty)$.) The map $A$ is called Fréchet differentiable at $\infty$ if $\left\|A(x)-A^{\prime}(\infty) x\right\|_{X}=o\left(\|x\|_{X}\right)$ as $\|x\|_{X} \rightarrow \infty$. The operator $A^{\prime}(\infty)$ is called Fréchet derivative of $A$ at $\infty$. (Be careful not to confuse the two concepts!)

Let $H$ be a Hilbert space with inner product $(\cdot, \cdot)_{H}$ and the induced norm $\|\cdot\|$, and let $X$ be a Banach space with norm $\|\cdot\|_{X}$, such that

(S) $X \subset H$ is dense in $H$ and the inclusion $X \hookrightarrow H$ is continuous (and hence we may assume $\|x\| \leq\|x\|_{X}$ for all $x \in X$ ).

In this paper for $R>0$ we write

$$
\begin{array}{ll}
B_{X}(\infty, R):=\left\{x \in X \mid\|x\|_{X}>R\right\}, & \bar{B}_{X}(\infty, R):=\left\{x \in X \mid\|x\|_{X} \geq R\right\}, \\
B_{H}(\infty, R):=\{x \in H \mid\|x\|>R\}, & \bar{B}_{H}(\infty, R):=\{x \in H \mid\|x\| \geq R\} .
\end{array}
$$

Let $V_{\infty}$ be an open neighbourhood of infinity in $H$. Then $V_{\infty} \cap X$ is open in $X$, and also star-shaped with respect to infinity provided $V_{\infty}$ star-shaped with respect to infinity. For clearness we shall write $V_{\infty} \cap X$ as $V_{\infty}^{X}$ when it is equipped with the induced topology from $X$.

Suppose that a functional $\mathcal{L}: V_{\infty} \rightarrow \mathbb{R}$ satisfies the following conditions:

$\left(\mathrm{F} 1_{\infty}\right) \mathcal{L}$ is continuous and continuously directional differentiable on $V_{\infty}$.

$\left(\mathrm{F} 2_{\infty}\right)$ There exists a continuous and continuously directional differentiable map $A: V_{\infty}^{X} \rightarrow X$, such that

$$
D \mathcal{L}(x)(u)=(A(x), u)_{H} \quad \text { for all } x \in V_{\infty} \cap X, \text { for all } u \in X .
$$

(This actually implies that $\left.\mathcal{L}\right|_{V_{\infty}^{X}} \in C^{1}\left(V_{\infty}^{X}, \mathbb{R}\right)$.)

$\left(\mathrm{F} 3_{\infty}\right)$ There exists a map $B$ from $\left(V_{\infty} \cap X\right) \cup\{\infty\}$ to the space $L_{s}(H)$ of bounded self-adjoint linear operators of $H$ such that $B(\infty)(X) \subset X$ and

$(D A(x)(u), v)_{H}=(B(x) u, v)_{H} \quad$ for all $x \in V_{\infty} \cap X$, for all $u, v \in X$.

(This implies: $D A(x)=\left.B(x)\right|_{X}$ for all $x \in V_{\infty} \cap X$, and thus $B(x)(X) \subset X$ for all $x \in\left(V_{\infty} \cap X\right) \cup\{\infty\}$.) 
$\left(\mathrm{C} 1_{\infty}\right)$ Either $0 \notin \sigma(B(\infty))$ or 0 is an isolated point of the spectrum $\left.\sigma(B(\infty)){ }^{1}\right)$.

$\left(\mathrm{C} 2_{\infty}\right)$ If $u \in H$ such that $B(\infty)(u)=v$ for some $v \in X$, then $u \in X$.

$\left(\mathrm{D}_{\infty}\right)$ The map $B:\left(V_{\infty} \cap X\right) \cup\{\infty\} \rightarrow L_{s}(H)$ has a decomposition

$$
B(x)=P(x)+Q(x) \text { for all } x \in\left(V_{\infty} \cap X\right) \cup\{\infty\},
$$

where $P(x): H \rightarrow H$ is a positive definite linear operator and $Q(x): H \rightarrow$ $H$ is a compact linear operator with the following properties:

$\left(D 1_{\infty}\right)$ All eigenfunctions of the operator $B(\infty)$ that correspond to negative eigenvalues belong to $X$;

$\left(\mathrm{D} 2_{\infty}\right)$ For any sequence $\left\{x_{k}\right\} \subset V_{\infty} \cap X$ with $\left\|x_{k}\right\| \rightarrow \infty$ it holds that $\left\|P\left(x_{k}\right) u-P(\infty) u\right\| \rightarrow 0$ for any $u \in H$

$\left(\mathrm{D} 3_{\infty}\right)$ The map $Q:\left(V_{\infty} \cap X\right) \cup\{\infty\} \rightarrow L(H)$ is continuous at $\infty$ with respect to the topology induced from $H$ on $V_{\infty} \cap X$, i.e. $\| Q(x)-$ $Q(\infty) \|_{L(H)} \rightarrow 0$ as $x \in V_{\infty} \cap X$ and $\|x\| \rightarrow \infty ;$

$\left(\mathrm{D} 4_{\infty}\right)$ For any sequence $\left\{x_{n}\right\} \subset V_{\infty} \cap X$ with $\left\|x_{n}\right\| \rightarrow \infty($ as $n \rightarrow \infty)$, there exist constants $C_{0}>0$ and $n_{0}>0$ such that

$\left(P\left(x_{n}\right) u, u\right)_{H} \geq C_{0}\|u\|^{2}$ for all $u \in H$, for all $n \geq n_{0}$.

As before let $H_{\infty}^{0}=\operatorname{Ker}(B(\infty))$, which is contained in $X$ by $\left(\mathrm{C} 2_{\infty}\right)$. Then $H_{\infty}^{ \pm}:=\left(H_{\infty}^{0}\right)^{\perp}$ is equal to the range of $B(\infty)$ by $\left(\mathrm{C} 1_{\infty}\right)$. (See Proposition B.2 in [13] and [14]). Obverse that $H_{\infty}^{ \pm}$splits as $H_{\infty}^{ \pm}=H_{\infty}^{+} \oplus H_{\infty}^{-}$, where $H_{\infty}^{+}$(resp. $H_{\infty}^{-}$) is positive (resp. negative) definite subspace of $B(\infty)$, that is, there exists some $a_{\infty}>0$ such that

$$
\begin{cases}\left(B(\infty) u^{+}, u^{+}\right)_{H} \geq 2 a_{\infty}\left\|u^{+}\right\|^{2} & \text { for all } u \in H_{\infty}^{+} \\ \left(B(\infty) u^{-}, u^{-}\right)_{H} \leq-2 a_{\infty}\left\|u^{-}\right\|^{2} & \text { for all } u \in H_{\infty}^{-}\end{cases}
$$

Write $X_{\infty}^{ \pm}:=H_{\infty}^{ \pm} \cap X$ and $X_{\infty}^{*}:=H_{\infty}^{*} \cap X, *=+,-$. We get topological direct sum decompositions $X=H_{\infty}^{0} \oplus X_{\infty}^{ \pm}$and $X_{\infty}^{ \pm}=X_{\infty}^{+} \oplus X_{\infty}^{-}$. In addition, $H_{\infty}^{0}$ and $X_{\infty}^{-}$have finite dimensions by $\left(\mathrm{D}_{\infty}\right)$. (Note: As in the proof of [13, Lemma 2.13] or [14, Lemma 3.1] the condition $H_{\infty}^{0} \subset X$ is enough for the following Lemmas 2.2 and 2.3 because this implies that $H_{\infty}^{0} \subset X$ is complete in both $H$ and $X$ and therefore that $H$ and $X$ induce equivalent norms on $H_{\infty}^{0}$ in the case). Let

$$
\nu_{\infty}:=\operatorname{dim} H_{\infty}^{0} \quad \text { and } \quad \mu_{\infty}:=\operatorname{dim} H_{\infty}^{-} .
$$

They are called the nullity and Morse index of $\mathcal{L}$ at infinity, respectively. Denote by $P_{\infty}^{*}$ the orthogonal projections from $H$ onto $H_{\infty}^{*}, *=+, 0,-$.

As in the proof of [13, Lemma 2.13] or [14, Lemma 3.1] we get that

$$
\left.B(\infty)\right|_{X_{\infty}^{ \pm}}: X_{\infty}^{ \pm} \rightarrow X_{\infty}^{ \pm}
$$

$\left({ }^{1}\right)$ The claim is actually implied in the following condition $\left(\mathrm{D}_{\infty}\right)$ by Proposition B.2 in [13] and [14]. In order to state some results without the condition $\left(\mathrm{D}_{\infty}\right)$ we still list it here. 
is a Banach space isomorphism. Let

$$
C_{1}^{\infty}=\left\|\left(\left.B(\infty)\right|_{X_{\infty}^{ \pm}}\right)^{-1}\right\|_{L\left(X_{\infty}^{ \pm}, X_{\infty}^{ \pm}\right)} \quad \text { and } \quad C_{2}^{\infty}=\left\|I-P_{\infty}^{0}\right\|_{L\left(X, X_{\infty}^{ \pm}\right)} .
$$

We shall give our results in cases $\nu_{\infty}>0$ and $\nu_{\infty}=0$, respectively. For the former case we further assume the following condition to be satisfied.

$\left(\mathrm{E}_{\infty}\right) M(A):=\lim _{R \rightarrow \infty} \sup \left\{\left\|\left(I-P_{\infty}^{0}\right) A(z)\right\|_{X}: z \in H_{\infty}^{0},\|z\|_{X} \geq R\right\}<\infty$, and there exist $R_{1}>0, \kappa>1$ and $\rho_{A} \in\left((\kappa /(\kappa-1)) C_{1}^{\infty} M(A), \infty\right)$, such that

$$
\begin{array}{r}
\left\|\left(I-P_{\infty}^{0}\right) A\left(z_{1}+x_{1}\right)-B(\infty) x_{1}-\left(I-P_{\infty}^{0}\right) A\left(z_{2}+x_{2}\right)+B(\infty) x_{2}\right\|_{X_{\infty}^{ \pm}} \\
\leq \frac{1}{\kappa C_{1}^{\infty}}\left\|z_{1}+x_{1}-z_{2}-x_{2}\right\|_{X}
\end{array}
$$

for all $x_{i} \in B_{X}\left(\theta, \rho_{A}\right) \cap X_{\infty}^{ \pm}$and $z_{i} \in H_{\infty}^{0}$ with $\left\|z_{i}\right\| \geq R_{1}, i=1,2$. Moreover, if (1.2) holds with $\rho_{A}=\infty$ the assumption that $M(A)<\infty$ is not needed. (Obverse that (1.2) is satisfied if

$$
\begin{aligned}
\| A\left(z_{1}+x_{1}\right)-A\left(z_{2}+x_{2}\right)-B(\infty)\left(x_{1}\right. & \left.-x_{2}\right) \|_{X} \\
& \leq \frac{1}{\kappa C_{1}^{\infty} C_{2}^{\infty}}\left\|z_{1}+x_{1}-z_{2}-x_{2}\right\|_{X}
\end{aligned}
$$

for all $x_{i} \in B_{X}\left(\theta, \rho_{A}\right) \cap X_{\infty}^{ \pm}$and $z_{i} \in H_{\infty}^{0}$ with $\left.\left\|z_{i}\right\| \geq R_{1}, i=1,2.\right)\left({ }^{2}\right)$

Clearly, $\left(\mathrm{E}_{\infty}\right)$ is satisfied if the following assumption holds.

$\left(\mathrm{SE}_{\infty}\right) M(A):=\lim _{R \rightarrow \infty} \sup \left\{\left\|\left(I-P_{\infty}^{0}\right) A(z)\right\|_{X}: z \in H_{\infty}^{0},\|z\|_{X} \geq R\right\}<\infty$, and there exists $\rho_{A} \in\left(C_{1}^{\infty} M(A), \infty\right)$ such that

$$
\frac{\left\|\left(I-P_{\infty}^{0}\right) A\left(z_{1}+x_{1}\right)-B(\infty) x_{1}-\left(I-P_{\infty}^{0}\right) A\left(z_{2}+x_{2}\right)+B(\infty) x_{2}\right\|_{X_{\infty}^{ \pm}}}{\left\|z_{1}+x_{1}-z_{2}-x_{2}\right\|_{X}} \rightarrow 0
$$

uniformly in $x_{1}, x_{2} \in B_{X}\left(\theta, \rho_{A}\right) \cap X_{\infty}^{ \pm}$as $\left(z_{1}, z_{2}\right) \in H_{\infty}^{0} \times H_{\infty}^{0}$ and $\left(\left\|z_{1}\right\|,\left\|z_{2}\right\|\right) \rightarrow(\infty, \infty)$. (Note: $\rho_{A}>(\kappa /(\kappa-1)) C_{1}^{\infty} M(A)$ if $\kappa>1$ is large enough.) Moreover, if this holds with $\rho_{A}=\infty$ the assumption that $M(A)<\infty$ is not needed.

Note: Since the norms $\|\cdot\|$ and $\|\cdot\|_{X}$ are equivalent on $H_{\infty}^{0}$ and we have assumed $\|u\| \leq\|u\|_{X}$ for all $u \in X$, which implies $\|z+x\|_{X}^{2} \geq\|z+x\|^{2}=$ $\|z\|^{2}+\|x\|^{2} \geq\|z\|^{2}$ for any $(z, x) \in H_{\infty}^{0} \times X_{\infty}^{ \pm}$, if $\left.B(\infty)\right|_{X} \in L(X)$ and $A$ has the strict Fréchet derivative $\left.B(\infty)\right|_{X}$ at $\infty$, it is easily proved that $\left(\mathrm{SE}_{\infty}\right)$ holds for any $\rho_{A} \in(0, \infty]$.

The following assumption is slightly weaker than $\left(\mathrm{E}_{\infty}\right)$.

$\left(\mathrm{E}_{\infty}^{\prime}\right) M(A):=\lim _{R \rightarrow \infty} \sup \left\{\left\|\left(I-P_{\infty}^{0}\right) A(z)\right\|_{X}: z \in H_{\infty}^{0},\|z\|_{X} \geq R\right\}<\infty$, and there exist $R_{1}>0, \kappa>1$ and $\rho_{A} \in\left((\kappa /(\kappa-1)) C_{1}^{\infty} M(A), \infty\right)$ such that

$\left({ }^{2}\right)$ If $R_{1}>0$ is large enough then $z+x \in V_{\infty} \cap X$ for any $z \in B_{H_{\infty}^{0}}\left(\infty, R_{1}\right)$ and any $x \in X_{\infty}^{ \pm}$. 


$$
\begin{array}{r}
\left\|\left(I-P_{\infty}^{0}\right) A\left(z+x_{1}\right)-B(\infty) x_{1}-\left(I-P_{\infty}^{0}\right) A\left(z+x_{2}\right)+B(\infty) x_{2}\right\|_{X_{\infty}^{ \pm}} \\
\leq \frac{1}{\kappa C_{1}^{\infty}}\left\|x_{1}-x_{2}\right\|_{X}
\end{array}
$$

holds for all $x_{i} \in B_{X}\left(\theta, \rho_{A}\right) \cap X_{\infty}^{ \pm}$and $z \in H_{\infty}^{0}$ with $\|z\| \geq R_{1}$. Moreover, if (1.4) holds with $\rho_{A}=\infty$ the assumption that $M(A)<\infty$ is not needed. (Clearly, (1.4) is satisfied if (1.3) is satisfied for all $x_{i} \in B_{X}\left(\theta, \rho_{A}\right) \cap X_{\infty}^{ \pm}$and $z_{1}=z_{2} \in H_{\infty}^{0}$ with $\left.\left\|z_{i}\right\| \geq R_{1}.\right)$

As above $\left(\mathrm{E}_{\infty}^{\prime}\right)$ is satisfied under the following assumption.

$\left(\mathrm{SE}_{\infty}^{\prime}\right) M(A):=\lim _{R \rightarrow \infty} \sup \left\{\left\|\left(I-P_{\infty}^{0}\right) A(z)\right\|_{X}: z \in H_{\infty}^{0},\|z\|_{X} \geq R\right\}<\infty$, and there exists $\rho_{A} \in\left(C_{1}^{\infty} M(A), \infty\right)$ such that

$$
\frac{\left\|\left(I-P_{\infty}^{0}\right) A\left(z+x_{1}\right)-B(\infty) x_{1}-\left(I-P_{\infty}^{0}\right) A\left(z+x_{2}\right)+B(\infty) x_{2}\right\|_{X_{\infty}^{ \pm}}}{\left\|x_{1}-x_{2}\right\|_{X}} \rightarrow 0
$$

uniformly in $x_{1}, x_{2} \in B_{X}\left(\theta, \rho_{A}\right) \cap X_{\infty}^{ \pm}$as $z \in H_{\infty}^{0}$ and $\|z\| \rightarrow \infty$.

(Note: $\rho_{A}>\left(\kappa /(\kappa-1) C_{1}^{\infty} M(A)\right.$ if $\kappa>1$ is large enough.) Moreover, if this holds with $\rho_{A}=\infty$ the assumption that $M(A)<\infty$ is not needed.

[Note: If $\left.B(\infty)\right|_{X} \in L(X)$ and $A$ has the strict Fréchet derivative $\left.B(\infty)\right|_{X}$ at $\infty$, then $\left(\mathrm{SE}_{\infty}^{\prime}\right)$ holds for any $\rho_{A} \in(0, \infty]$.]

We have the following splitting lemmas at infinity on Hilbert spaces.

THEOREM 1.1. Under the above assumptions $(\mathrm{S}),\left(\mathrm{F} 1_{\infty}\right)-\left(\mathrm{F} 3_{\infty}\right)$ and $\left(\mathrm{C} 1_{\infty}\right)-$ $\left(\mathrm{C} 2_{\infty}\right),\left(\mathrm{D}_{\infty}\right)$, also suppose that $\nu_{\infty}>0$ and $\left(\mathrm{E}_{\infty}^{\prime}\right)$ is satisfied and that

$$
\mathcal{L}(u)=\frac{1}{2}(B(\infty) u, u)_{H}+o\left(\|u\|^{2}\right) \quad \text { as }\|u\| \rightarrow \infty .\left({ }^{3}\right)
$$

Then there exist a positive number $R$, a (unique) continuous map $h^{\infty}: B_{H_{\infty}^{0}}(\infty, R)$ $\rightarrow X_{\infty}^{ \pm}$(which takes values in $\bar{B}_{X_{\infty}^{ \pm}}\left(\theta, \rho_{A}\right)$ in the case $\left.M(A)<\infty\right)$ satisfying

$$
\left(I-P_{\infty}^{0}\right) A\left(z+h^{\infty}(z)\right)=0 \quad \text { for all } z \in \bar{B}_{H_{\infty}^{0}}(\infty, R),
$$

and a homeomorphism $\Phi: B_{H_{\infty}^{0}}(\infty, R) \oplus H_{\infty}^{ \pm} \rightarrow B_{H_{\infty}^{0}}(\infty, R) \oplus H_{\infty}^{ \pm}$of form

$$
\Phi\left(z+u^{+}+u^{-}\right)=z+h^{\infty}(z)+\phi_{z}\left(u^{+}+u^{-}\right)
$$

with $\phi_{z}\left(u^{+}+u^{-}\right) \in H_{\infty}^{ \pm}$and $\Phi\left(B_{H_{\infty}^{0}}(\infty, R) \oplus X_{\infty}^{ \pm}\right) \subset X$, such that

$$
\mathcal{L} \circ \Phi\left(z+u^{+}+u^{-}\right)=\left\|u^{+}\right\|^{2}-\left\|u^{-}\right\|^{2}+\mathcal{L}\left(z+h^{\infty}(z)\right)
$$

for all $\left(z, u^{+}+u^{-}\right) \in B_{H_{\infty}^{0}}(\infty, R) \times H_{\infty}^{ \pm}$. The homeomorphism $\Phi$ has also properties:

(a) For each $z \in B_{H_{\infty}^{0}}(\infty, R), \Phi(z, \theta)=z+h^{\infty}(z)$, and $\phi_{z}\left(u^{+}+u^{-}\right) \in H_{\infty}^{-}$ if and only if $u^{+}=\theta$;

$\left({ }^{3}\right)$ This condition is weaker than the assumption $\left(\mathrm{A}_{\infty}\right)$ in [2]. See Section 3.1 below. 
(b) The restriction of $\Phi$ to $B_{H_{\infty}^{0}}(\infty, R) \oplus H_{\infty}^{-}$is a homeomorphism from $B_{H_{\infty}^{0}}(\infty, R) \oplus H_{\infty}^{-} \subset X$ onto $\left.\Phi\left(B_{H_{\infty}^{0}}(\infty, R) \oplus H_{\infty}^{-}\right)\right) \subset X$ even if the topologies on these two sets are chosen as the induced one by $X$.

The map $h^{\infty}$ and the function $\mathcal{L}^{\infty}: B_{H_{\infty}^{0}}(\infty, R) \rightarrow \mathbb{R}, z \mapsto \mathcal{L}\left(z+h^{\infty}(z)\right)$ also satisfy:

(i) $\lim _{\|z\|_{X} \rightarrow \infty}\left\|h^{\infty}(z)\right\|_{X}=0$ provided that

$$
\lim _{R \rightarrow \infty} \sup \left\{\left\|\left(I-P_{\infty}^{0}\right) A(z)\right\|_{X}: z \in H_{\infty}^{0},\|z\|_{X} \geq R\right\}=0
$$

(ii) If $A$ is $C^{1}$, then $h^{\infty}$ is $C^{1}$ and

$$
\begin{aligned}
& d h^{\infty}(z)=-\left.\left[\left.\left(I-P_{\infty}^{0}\right) A^{\prime}\left(z+h^{\infty}(z)\right)\right|_{X_{\infty}^{ \pm}}\right]^{-1}\left(I-P_{\infty}^{0}\right) A^{\prime}\left(z+h^{\infty}(z)\right)\right|_{H_{\infty}^{0}}, \\
& \text { moreover the function } \mathcal{L}^{\infty} \text { is } C^{2} \text { and }
\end{aligned}
$$

(1.9) $d \mathcal{L}^{\infty}\left(z_{0}\right)(z)=\left(A\left(z_{0}+h^{\infty}\left(z_{0}\right)\right), z\right)_{H}, \quad$ for all $z_{0} \in B_{H_{\infty}^{0}}(\infty, R), z \in H_{\infty}^{0}$.

(iii) If $\mathcal{L}$ is $C^{2}$ then $h^{\infty}$ is also $C^{1}$ as a map to $H_{\infty}^{ \pm}$(hence $\left.X_{\infty}^{ \pm}\right)$.

If $\left(\mathrm{E}_{\infty}^{\prime}\right)$ is replaced by the slightly strong $\left(\mathrm{E}_{\infty}\right)\left(\right.$ and $\rho_{A}$ is given by $\left(\mathrm{E}_{\infty}\right)$ ) one has:

(iv) The map $h^{\infty}$ is Lipschitz, and has a strict Fréchet derivative zero at $\infty$;

(v) $\mathcal{L}^{\infty}$ is $C^{1}$ and (1.9) holds;

(vi) If $B(\infty) \in L(X)$ and $A$ has a strict Fréchet derivative $\left.B(\infty)\right|_{X}$ at $\infty$, then $\mathcal{L}^{\infty}$ is $C^{2-0}$ and $d \mathcal{L}^{\infty}$ has the strict Fréchet derivative zero at $\infty$. (In this case, as noted below $\left(\mathrm{SE}_{\infty}\right)$ we may choose $\rho_{A}$ above to be any positive number, but $R$ depends on this choice.)

REMARK 1.2. Similar conclusions to Remarks 2.2, 2.3 in [13], [14] also hold. Namely, we only use Lemmas 2.4 and 2.5 in the proof of Lemma 2.6. Hence the condition $\left(\mathrm{D}_{\infty}\right)$ can be replaced by the following:

$\left(\mathrm{D}_{\infty}^{\prime}\right)$ There exist a subset $U_{\infty} \subset V_{\infty}$ of form $U_{\infty}=\bar{B}_{H_{\infty}^{0}}\left(\infty, R^{\prime}\right) \oplus H_{\infty}^{ \pm}$, a positive number $c_{\infty}$ and a function $\omega_{\infty}: U_{\infty} \cap X \rightarrow[0, \infty)$ with the property that $\omega_{\infty}(x) \rightarrow 0$ as $x \in U_{\infty} \cap X$ and $\|x\| \rightarrow \infty$, such that

$\left(\mathrm{D}_{\infty 1}^{\prime}\right)$ the kernel $H_{\infty}^{0}$ and negative definite subspace $H_{\infty}^{-}$of $B(\infty)$ are finite dimensional subspaces contained in $X$;

$\left(\mathrm{D}_{\infty 2}^{\prime}\right)(B(x) v, v)_{H} \geq c_{\infty}\|v\|^{2}$ for all $v \in H_{\infty}^{+}, x \in U_{\infty} \cap X$;

$\left(\mathrm{D}_{\infty 3}^{\prime}\right)\left|(B(x) u, v)_{H}-(B(\infty) u, v)_{H}\right| \leq \omega_{\infty}(x)\|u\| \cdot\|v\|$ for all $u \in H, v \in$ $H_{\infty}^{-} \oplus H_{\infty}^{0}$

$\left(\mathrm{D}_{\infty 4}^{\prime}\right)(B(x) u, u)_{H} \leq-c_{\infty}\|u\|^{2}$ for all $u \in H_{\infty}^{-}, x \in U_{\infty} \cap X$.

In order to state our second result, for positive numbers $R$ and $\delta$ we set

$$
C_{R, \delta}:=B_{H_{\infty}^{0}}(\infty, R) \oplus B_{H_{\infty}^{+}}(\theta, \delta) \oplus B_{H_{\infty}^{-}}(\theta, \delta) .
$$

(It is often identified with $B_{H_{\infty}^{0}}(\infty, R) \times B_{H_{\infty}^{+}}(\theta, \delta) \times B_{H_{\infty}^{-}}(\theta, \delta)$ ). 
THEOREM 1.3. Under the above assumptions $(\mathrm{S}),\left(\mathrm{F} 1_{\infty}\right)-\left(\mathrm{F} 3_{\infty}\right)$ and $\left(\mathrm{C} 1_{\infty}\right)-$ $\left(\mathrm{C} 2_{\infty}\right),\left(\mathrm{D}_{\infty}\right)$, also suppose that $\nu_{\infty}>0$ and $\left(\mathrm{E}_{\infty}^{\prime}\right)$ is satisfied. Then for any $r \in(0, \infty)$ there exist positive numbers $R, \delta_{r}>0$ and a (unique) continuous map $h^{\infty}: B_{H_{\infty}^{0}}(\infty, R) \rightarrow X_{\infty}^{ \pm}$(which takes values in $\bar{B}_{X_{\infty}^{ \pm}}\left(\theta, \rho_{A}\right)$ in the case $M(A)<\infty)$ satisfying

$$
\left(I-P_{\infty}^{0}\right) A\left(z+h^{\infty}(z)\right)=0 \quad \text { for all } z \in B_{H_{\infty}^{0}}(\infty, R),
$$

an open set $V(R, r)$ in $H$ with $V(R, r) \subset \overline{C_{R, r+\rho_{A}}}$, and a homeomorphism $\Phi: C_{R, \delta_{r}} \rightarrow V(R, r)$ of form

$$
\Phi\left(z+u^{+}+u^{-}\right)=z+h^{\infty}(z)+\phi_{z}\left(u^{+}+u^{-}\right)
$$

with $\phi_{z}\left(u^{+}+u^{-}\right) \in H_{\infty}^{ \pm}$and $\Phi\left(C_{R, \delta_{r}} \cap X\right) \subset X$, such that

$$
\mathcal{L} \circ \Phi\left(z, u^{+}+u^{-}\right)=\left\|u^{+}\right\|^{2}-\left\|u^{-}\right\|^{2}+\mathcal{L}\left(z+h^{\infty}(z)\right)
$$

for all $\left(z, u^{+}, u^{-}\right) \equiv z+u^{+}+u^{-} \in C_{R, \delta_{r}}$. The homeomorphism $\Phi$ also possesses properties:

(a) For each $z \in B_{H_{\infty}^{0}}(\infty, R), \Phi(z, \theta)=z+h^{\infty}(z), \phi_{z}\left(u^{+}+u^{-}\right) \in H_{\infty}^{-}$if and only if $u^{+}=\theta$;

(b) The restriction of $\Phi$ to $B_{H_{\infty}^{0}}(\infty, R) \oplus B_{H_{\infty}^{-}}\left(\theta, \delta_{r}\right)$ is a homeomorphism from $B_{H_{\infty}^{0}}(\infty, R) \oplus B_{H_{\infty}^{-}}\left(\theta, \delta_{r}\right) \subset X$ onto $\Phi\left(B_{H_{\infty}^{0}}(\infty, R) \oplus B_{H_{\infty}^{-}}\left(\theta, \delta_{r}\right)\right) \subset$ $X$ even if the topologies on these two sets are chosen as the induced one by $X$.

The map $h^{\infty}$ and the function $\mathcal{L}^{\infty}: B_{H_{\infty}^{0}}(\infty, R) \rightarrow \mathbb{R}, z \mapsto \mathcal{L}\left(z+h^{\infty}(z)\right)$ satisfy the conclusions (i)-(iii) in Theorem 1.1, and also (iv)-(vi) in Theorem 1.1 if $\left(\mathrm{E}_{\infty}\right)$ holds and $\rho_{A}$ is given by $\left(\mathrm{E}_{\infty}\right)$.

In Theorems 1.1, 1.3, if $\mathcal{L}$ is $C^{2}$ and $D^{2} \mathcal{L}(w)=B(\infty)+o(1)$ as $\|w\| \rightarrow \infty$, we shall prove in Remark 2.15 that $\Phi^{-1}$ is $C^{1}$ outside the submanifold of codimension $\mu_{\infty}$ if $R>0$ is large enough.

REMARK 1.4. Similar conclusions to Remarks 2.2, 2.3 in [13], [14] also hold. By the note below Lemma 2.5, we can still get Theorem 1.3 if we replace the condition $\left(\mathrm{D}_{\infty}\right)$ by the following:

$\left(\mathrm{D}_{\infty}^{\prime \prime}\right)$ There exist a subset of $X$ of form

$$
W_{\infty}=\bar{B}_{H_{\infty}^{0}}\left(\infty, R^{\prime}\right) \oplus\left(\bar{B}_{H}\left(\theta, r^{\prime}\right) \cap X_{\infty}^{ \pm}\right) \subset V_{\infty} \cap X
$$

a positive number $c_{\infty}$ and a function $\omega_{\infty}: W_{\infty} \rightarrow[0, \infty)$ with the property that $\omega_{\infty}(x) \rightarrow 0$ as $x \in W_{\infty}$ and $\|x\| \rightarrow \infty$, such that

$\left(\mathrm{D}_{\infty 1}^{\prime \prime}\right)$ the kernel $H_{\infty}^{0}$ and negative definite subspace $H_{\infty}^{-}$of $B(\infty)$ are finite dimensional subspaces contained in $X$;

$\left(\mathrm{D}_{\infty 2}^{\prime \prime}\right)(B(x) v, v)_{H} \geq c_{\infty}\|v\|^{2}$ for all $v \in H_{\infty}^{+}, x \in W_{\infty}$; 
$\left(\mathrm{D}_{\infty 3}^{\prime \prime}\right)\left|(B(x) u, v)_{H}-(B(\infty) u, v)_{H}\right| \leq \omega_{\infty}(x)\|u\| \cdot\|v\|$ for all $u \in H, v \in$ $H_{\infty}^{-} \oplus H_{\infty}^{0}$

$\left(\mathrm{D}_{\infty 4}^{\prime \prime}\right)(B(x) u, u)_{H} \leq-c_{\infty}\|u\|^{2}$ for all $u \in H_{\infty}^{-}, x \in W_{\infty}$.

Corollary 1.5. Suppose that one of the following condition groups holds:

(a) $(\mathrm{S}),\left(\mathrm{F} 1_{\infty}\right)-\left(\mathrm{F} 3_{\infty}\right)$ and $\left(\mathrm{C} 1_{\infty}\right)-\left(\mathrm{C} 2_{\infty}\right),\left(\mathrm{D}_{\infty}\right)$ and $\left(\mathrm{E}_{\infty}\right)$;

(b) $(\mathrm{S}),\left(\mathrm{F} 1_{\infty}\right)-\left(\mathrm{F} 3_{\infty}\right)$ and $\left(\mathrm{C} 1_{\infty}\right)-\left(\mathrm{C} 2_{\infty}\right),\left(\mathrm{D}_{\infty}\right)$ and $\left(\mathrm{E}_{\infty}^{\prime}\right)$, and $A$ being $C^{1}$. Then each critical point $z$ of the function $\mathcal{L}^{\infty}: B_{H_{\infty}^{0}}(\infty, R) \rightarrow \mathbb{R}$ gives a critical point of $\mathcal{L}, z+h^{\infty}(z)$.

Proof. Under the condition group (a) or (b), $\mathcal{L}^{\infty}$ is at least $C^{1}$. For a critical point $z$ of it $(1.9)$ shows that $\left(A\left(z+h^{\infty}(z)\right), z^{\prime}\right)_{H}=0$ for all $z^{\prime} \in H_{\infty}^{0}$, i.e.

$$
\left(P_{\infty}^{0} A\left(z+h^{\infty}(z)\right), u\right)_{H}=0 \quad \text { for all } u \in H .
$$

This and (1.10) imply $A\left(z+h^{\infty}(z)\right)=\theta$. Since $X$ is dense in $H$, the desired claim follows from the condition $\left(\mathrm{F} 22_{\infty}\right)$.

When $X=H$, Theorems 1.1, 1.3 have the following corollaries, respectively.

Corollary 1.6. Let $V_{\infty}$ be a neighbourhood of infinity in a Hilbert space $H$, and let $\mathcal{L}: V_{\infty} \rightarrow \mathbb{R}$ be a $C^{1}$-functional. Suppose that $\nabla \mathcal{L}: V_{\infty} \rightarrow H$ is continuously directional differentiable and that there exists a map $B$ from $V_{\infty} \cup\{\infty\}$ to the space $L_{s}(H)$ of bounded self-adjoint linear operators of $H$ such that

$$
(D \nabla \mathcal{L}(x)(u), v)_{H}=(B(x) u, v)_{H} \quad \text { for all } x \in V_{\infty}, \text { for all } u, v \in H .
$$

$\left(\right.$ So $\mathcal{L}$ has the Gâteaux derivative of second order $\mathcal{L}^{\prime \prime}(x)=B(x)$ at $x \in V_{\infty}$.) Write $\mathcal{L}$ as

$$
\mathcal{L}(x)=\frac{1}{2}(B(\infty) x, x)_{H}+g(x) .
$$

( $g$ has the Gâteaux derivative of second order $g^{\prime \prime}(x)=B(x)-B(\infty)$ at $x \in V_{\infty}$.) Suppose

(a) $g(x)=o\left(\|x\|^{2}\right)$ as $\|x\| \rightarrow \infty$;

(b) $0 \in \sigma(B(\infty))$ and $B(\infty)=P(\infty)+Q(\infty)$, where $P(\infty) \in \mathcal{L}_{s}(H)$ is positive definite and $Q(\infty) \in \mathcal{L}_{s}(H)$ is compact;

(c) For any sequence $\left\{x_{n}\right\} \subset V_{\infty}$ with $\left\|x_{n}\right\| \rightarrow \infty($ as $n \rightarrow \infty)$, there exist constants $C_{0}>0$ and $n_{0}>0$ such that

$\left(\left[B\left(x_{n}\right)-Q(\infty)\right] u, u\right)_{H} \geq C_{0}\|u\|^{2} \quad$ for all $u \in H$, for all $n \geq n_{0}$.

(d) $H_{\infty}^{0}:=\operatorname{Ker}(B(\infty)) \neq\{\theta\}, H_{\infty}^{ \pm}:=\left(H_{\infty}^{0}\right)^{\perp}, C_{1}^{\infty}=\left\|\left(\left.B(\infty)\right|_{H_{\infty}^{ \pm}}\right)^{-1}\right\|_{L\left(H_{\infty}^{ \pm}\right)}$, if $M(A):=\lim _{R \rightarrow \infty} \sup \left\{\left\|\left(I-P_{\infty}^{0}\right) A(z)\right\|: z \in H_{\infty}^{0},\|z\| \geq R\right\}<\infty$ with $A=\nabla \mathcal{L}$, there exist constants $R_{1}>0, \kappa>1, \rho_{A} \in(\kappa /(\kappa-1))\left(C_{1}^{\infty} M(A), \infty\right)$ such that for all $y \in B_{H_{\infty}^{ \pm}}\left(\theta, \rho_{A}\right), z \in \bar{B}_{H_{\infty}^{0}}\left(\theta, R_{1}\right)$,

$$
\left\|\left.\left(I-P_{\infty}^{0}\right)[B(z+y)-B(\infty)]\right|_{H_{\infty}^{ \pm}}\right\|_{L\left(H_{\infty}^{ \pm}\right)} \leq \frac{1}{\kappa C_{1}^{\infty}} .
$$


Moreover, that $M(A)<\infty$ is not needed if there exists a constant $R_{1}>0$, $\kappa>1$ such that for all $y \in H_{\infty}^{ \pm}, z \in \bar{B}_{H_{\infty}^{0}}\left(\theta, R_{1}\right)$,

$$
\left\|\left.\left(I-P_{\infty}^{0}\right)[B(z+y)-B(\infty)]\right|_{H_{\infty}^{ \pm}}\right\|_{L\left(H_{\infty}^{ \pm}\right)} \leq \frac{1}{\kappa C_{1}^{\infty}} .
$$

Then there exist a positive number $R \geq R_{1}$, a (unique) continuous map

$$
h^{\infty}: B_{H_{\infty}^{0}}(\infty, R) \rightarrow H_{\infty}^{ \pm}
$$

satisfying (1.6) with $A=\nabla \mathcal{L}$, which takes values in $\bar{B}_{H_{\infty}^{ \pm}}\left(\theta, \rho_{A}\right)$ in the case $M(A)<\infty$, and a homeomorphism $\Phi: B_{H_{\infty}^{0}}(\infty, R) \oplus H_{\infty}^{ \pm} \rightarrow B_{H_{\infty}^{0}}(\infty, R) \oplus H_{\infty}^{ \pm}$ such that

$$
\mathcal{L} \circ \Phi\left(z+u^{+}+u^{-}\right)=\left\|u^{+}\right\|^{2}-\left\|u^{-}\right\|^{2}+\mathcal{L}\left(z+h^{\infty}(z)\right)
$$

for all $\left(z, u^{+}+u^{-}\right) \in B_{H_{\infty}^{0}}(\infty, R) \times H_{\infty}^{ \pm}$. Moreover, if $\mathcal{L}$ is $C^{2}$ then the map $h^{\infty}$ is $C^{1}$ and the function $B_{H_{\infty}^{0}}(\infty, R) \rightarrow \mathbb{R}, z \mapsto \mathcal{L}^{\infty}(z):=\mathcal{L}\left(z+h^{\infty}(z)\right)$ is $C^{2}$.

Proof. By Propositions B.2 and B.3 in [13] or [14], 0 is an isolated spectrum point of $B(\infty)$ ), and $B(\infty)$ has the finite dimensional kernel $H_{\infty}^{0}$ and negative definite subspace $H_{\infty}^{-}$. For $x \in V_{\infty}$ let $P(x)=P(\infty)+g^{\prime \prime}(x)=B(x)-Q(\infty)$ and $Q(x) \equiv Q(\infty)$. Then $B(x)=P(x)+Q(x)$. The condition (iii) implies that $\left(\mathrm{D} 4_{\infty}\right)$ is satisfied. It follows that $P(x)$ is positive definite for each $x$ in a neighbourhood of infinity in $H$. Hence $\left(\mathrm{D}_{\infty}\right)$ is satisfied.

Next we shows that the condition (iv) implies $\left(\mathrm{E}_{\infty}^{\prime}\right)$. Since $g^{\prime}(x)=A(x)-$ $B(\infty) x$ with $A=\nabla \mathcal{L}$, and $g^{\prime \prime}(x)=B(x)-B(\infty)$ using the mean value theorem in inequality form we deduce that

$$
\begin{aligned}
& \frac{\left\|\left(I-P_{\infty}^{0}\right) A\left(z+x_{1}\right)-B(\infty) x_{1}-\left(I-P_{\infty}^{0}\right) A\left(z+x_{2}\right)+B(\infty) x_{2}\right\|}{\left\|x_{1}-x_{2}\right\|} \\
& =\frac{\left\|\left(I-P_{\infty}^{0}\right) g^{\prime}\left(z+x_{1}\right)-\left(I-P_{\infty}^{0}\right) g^{\prime}\left(z+x_{2}\right)\right\|}{\left\|x_{1}-x_{2}\right\|} \\
& \leq \sup _{t \in[0,1]}\left\|\left.\left(I-P_{\infty}^{0}\right) g^{\prime \prime}\left(z+t x_{1}+(1-t) x_{2}\right)\right|_{H_{\infty}^{ \pm}}\right\|_{L\left(H_{\infty}^{ \pm}\right)} \leq \frac{1}{\kappa C_{1}^{\infty}}
\end{aligned}
$$

for all $z \in \bar{B}_{H_{\infty}^{0}}\left(\infty, R_{1}\right)$ and $x_{i} \in B_{H_{\infty}^{ \pm}}\left(\theta, \rho_{A}\right), i=1,2$ and $x_{1} \neq x_{2}$. Moreover, since $I-P_{\infty}^{0} \neq 0, C_{2}^{\infty}=\left\|I-P_{\infty}^{0}\right\|_{L\left(H, H_{\infty}^{ \pm}\right)}=1$. So the condition $\left(\mathrm{E}_{\infty}^{\prime}\right)$ holds. Corollary 1.6 immediately follows from Theorem 1.1.

In Corollary 1.6, if $\mathcal{L}$ is $C^{2}$ and $g^{\prime \prime}(x)=o(1)$ as $\|x\| \rightarrow \infty$ then the conditions (c)-(d) are satisfied automatically. This almost leads to the splitting lemmas at infinity first established by Thomas Bartsch and Shujie Li [2, p. 431]. See Section 3.1 below for a detailed explanation. As in the proof of Corollary 1.6 Theorem 1.3 leads to

COROLlary 1.7. Under the assumptions (b)-(d) of Corollary 1.6, for any $r \in(0, \infty)$ there exist positive numbers $R \geq R_{1}, \delta_{r}>0$ and a (unique) continuous 
map $h^{\infty}: B_{H_{\infty}^{0}}(\infty, R) \rightarrow X_{\infty}^{ \pm}$(which takes values in $\bar{B}_{X_{\infty}^{ \pm}}\left(\theta, \rho_{A}\right)$ in the case $M(A)<\infty)$ satisfying $(1.10)$ with $A=\nabla \mathcal{L}$, an open set $V(R, r)$ in $H$ with $V(R, r) \subset \overline{C_{R, r+\rho_{A}}}$, and a homeomorphism $\Phi: C_{R, \delta_{r}} \rightarrow V(R, r)$ such that

$$
\mathcal{L} \circ \Phi\left(z, u^{+}+u^{-}\right)=\left\|u^{+}\right\|^{2}-\left\|u^{-}\right\|^{2}+\mathcal{L}\left(z+h^{\infty}(z)\right)
$$

for all $\left(z, u^{+}, u^{-}\right) \equiv z+u^{+}+u^{-} \in C_{R, \delta_{r}}$. Moreover, if $\mathcal{L}$ is $C^{2}$ then the map $h^{\infty}$ is $C^{1}$ and the function $B_{H_{\infty}^{0}}(\infty, R) \ni z \mapsto \mathcal{L}\left(z+h^{\infty}(z)\right) \in \mathbb{R}$ is $C^{2}$.

This corollary generalizes not only a slightly different version of Bartsch-Li splitting lemmas at infinity [2] given in [10, Proposition 3.3] but also Theorem 2.1 in [5]. Moreover, we do not need the assumption (1.5). See Section 3.2 below for a detailed explanation.

The premise of the assumptions $\left(\mathrm{E}_{\infty}\right)$ and $\left.\left(\mathrm{E}_{\infty}^{\prime}\right)\right)$ is $\nu_{\infty}>0$. When $\nu_{\infty}=0$ the proofs of Theorems 1.1, 1.3 cannot be completed if no further conditions are imposed. The following may be viewed as a corresponding version of them in the case $\nu_{\infty}=0$.

THEOREM 1.8. Under the above assumptions $(\mathrm{S}),\left(\mathrm{F} 1_{\infty}\right)-\left(\mathrm{F} 3_{\infty}\right)$ and $\left(\mathrm{C} 1_{\infty}\right)$ $-\left(\mathrm{C} 2_{\infty}\right),\left(\mathrm{D}_{\infty}\right)$, also suppose that $\nu_{\infty}=0$ and that there exist constants $R>0$ and $\lambda \in\left(0, a_{\infty}\right)$ such that

$$
\begin{aligned}
& |\mathcal{L}(u)-(B(\infty) u, u) / 2| \leq \lambda\|u\|^{2} \quad \text { for all } u \in \bar{B}_{H}(\infty, R), \\
& \|A(u)-B(\infty) u\| \leq \lambda\|u\| \quad \text { for all } u \in \bar{B}_{H}(\infty, R) \cap X .
\end{aligned}
$$

(a) If $\mu_{\infty}^{-}=0$ then there exist a number $\mathfrak{R}>0$ and a homeomorphism $\phi$ from $B_{H}(\infty, \mathfrak{R})$ onto an open subset of $H$ to satisfy:

$$
\begin{aligned}
\mathcal{L}(\phi(u)) & =\|u\|^{2} & \text { for all } u & \in B_{H}(\infty, \mathfrak{R}), \\
\frac{\|u\|}{\sqrt{2 a_{\infty}}} \leq\|\phi(u)\| & \leq \frac{1}{\sqrt{a_{\infty}-\lambda}}\|u\| & \text { for all } u & \in B_{H}(\infty, \mathfrak{R}) .
\end{aligned}
$$

(b) If $\mu_{\infty}^{-}>0$ then there exist a number $\Re>0$ and a homeomorphism $\phi$ from $B_{H_{\infty}^{+}}(\infty, \mathfrak{R}) \oplus H_{\infty}^{-}$onto an open subset of $H$ such that for all $(u, v) \in$ $B_{H_{\infty}^{+}}(\infty, \mathfrak{R}) \times H_{\infty}^{-}$,

$$
\begin{gathered}
\mathcal{L}(\phi(u+v))=\|u\|^{2}-\|v\|^{2}, \\
\frac{\|u\|}{\sqrt{2\|B(\infty)\|}} \leq\left\|P_{\infty}^{+} \circ \phi(u+v)\right\| \leq \sqrt{a_{\infty}-\lambda}\|u\|, \\
P_{\infty}^{-} \circ \phi\left(B_{H_{\infty}^{+}}(\infty, \mathfrak{R}) \oplus H_{\infty}^{-}\right)=H_{\infty}^{-},
\end{gathered}
$$

where $P_{\infty}^{+}$and $P_{\infty}^{-}$are the orthogonal projections onto $H_{\infty}^{+}$and $H_{\infty}^{-}$, respectively. 
COROLlaRY 1.9. Under the above assumptions $(\mathrm{S}),\left(\mathrm{F} 1_{\infty}\right)-\left(\mathrm{F} 3_{\infty}\right)$ and $\left(\mathrm{C} 1_{\infty}\right)$ $-\left(\mathrm{C} 2_{\infty}\right),\left(\mathrm{D}_{\infty}\right)$, let $\nu_{\infty}=0,(1.5)$ hold and

$$
\|A(u)-B(\infty) u\|=o(\|u\|) \quad \text { as } u \in X \text { and }\|u\| \rightarrow \infty .
$$

Then the conclusions in Theorem 1.8 hold with $\lambda=a_{\infty} / 2$ and some $\mathfrak{R}>0$.

Perhaps, the condition (1.5) (resp. (1.11)) may be derived from (1.13) (resp. (1.12)). But the author does not know how to do.

One of main applications of the splitting lemmas at infinity is to compute the critical group at infinity of $\mathcal{L}, C_{*}(\mathcal{L}, \infty):=\lim _{\longleftarrow} H_{*}(H,\{\mathcal{L} \leq a\} ; \mathbb{F})$ the inverse limit of the system $\left\{H_{*}\left(H, \mathcal{L}^{a}\right) \rightarrow H_{*}\left(H, \mathcal{L}^{b}\right) \longleftarrow-\infty<a \leq b<\infty\right\}$, where the homomorphism $H_{*}\left(H, \mathcal{L}^{a}\right) \rightarrow H_{*}\left(H, \mathcal{L}^{b}\right)$ is induced by the inclusion $\left.\left(H, \mathcal{L}^{a}\right)\right) \hookrightarrow$ $\left.\left(H, \mathcal{L}^{b}\right)\right)$. In the case $\nu_{\infty}=0$ and $\mu_{\infty}>0$ it follows from (1.5) that $\mathcal{L}$ is bounded from below on $H_{\infty}^{+}$and that $\mathcal{L}(u) \rightarrow-\infty$ for $u \in H_{\infty}^{-}$as $\|u\| \rightarrow \infty$. By Proposition 3.8 of [2] we get that $C_{j}(\mathcal{L}, \infty)=\delta_{k j} \mathbb{F}$ for $k=\mu_{\infty}=\operatorname{dim} H_{\infty}^{-}$. If $\nu_{\infty}=\mu_{\infty}=0$ this also holds because $C_{*}(\mathcal{L}, \infty)=H_{*}(H,\{\|u\| \geq R\} ; \mathbb{F})$ for any sufficiently large $R>0$.

For Theorems 1.1, 1.3 and 1.8 we can also give a corresponding result with Theorem 2.25 of [13] or Theorem 6.1 of [14].

The proofs of Theorems 1.1, 1.3 and 1.8 will be given in Section 2. Some relations between these theorems and previous ones will be discussed in Section 3 . In Section 4, as a simple application we give a generalization of Theorem 5.2 in [2]. It shows that our results may give better results even if for $C^{2}$ functionals. Our theory can be used to deal with a class of more general functionals of form $J(u)=$ $\int_{\Omega} F(x, u(x), \nabla u(x)) d x$ (with lower smoothness than $C^{2}$ usually), see [13]-[15].

\section{Proofs of main theorems}

For reader's conveniences we here state the following parameterized version of Theorem 1.1 in [9]. Its proof was given in Appendix A of [13] and [14].

TheOrem 2.1. Let $(H,\|\cdot\|)$ be a normed vector space and let $\Lambda$ be a compact topological space. Let $J: \Lambda \times B_{H}(\theta, 2 \delta) \rightarrow \mathbb{R}$ be continuous, and for every $\lambda \in \Lambda$ the function $J(\lambda, \cdot): B_{H}(\theta, 2 \delta) \rightarrow \mathbb{R}$ is continuously directional differentiable. Assume that there exist a closed vector subspace $H^{+}$and a finite-dimensional vector subspace $H^{-}$of $H$ such that $H^{+} \oplus H^{-}$is a direct sum decomposition of $H$ and

(a) $J(\lambda, \theta)=0$ and $D_{2} J(\lambda, \theta)=0$,

(b) $\left[D_{2} J\left(\lambda, x+y_{2}\right)-D_{2} J\left(\lambda, x+y_{1}\right)\right]\left(y_{2}-y_{1}\right)<0$ for any $(\lambda, x) \in \Lambda \times$ $\bar{B}_{H^{+}}(\theta, \delta), y_{1}, y_{2} \in \bar{B}_{H^{-}}(\theta, \delta)$ and $y_{1} \neq y_{2}$,

(c) $D_{2} J(\lambda, x+y)(x-y)>0$ for any $(\lambda, x, y) \in \Lambda \times \bar{B}_{H^{+}}(\theta, \delta) \times \bar{B}_{H^{-}}(\theta, \delta)$ and $(x, y) \neq(\theta, \theta)$, 
(d) $D_{2} J(\lambda, x) x>p(\|x\|)$ for any $(\lambda, x) \in \Lambda \times \bar{B}_{H^{+}}(\theta, \delta) \backslash\{\theta\}$, where $p:(0, \delta]$ $\rightarrow(0, \infty)$ is a non-decreasing function.

Then there exist a positive $\varepsilon \in \mathbb{R}$, an open neighbourhood $U$ of $\Lambda \times\{\theta\}$ in $\Lambda \times H$ and a homeomorphism

$$
\phi: \Lambda \times\left(B_{H^{+}}(\theta, \sqrt{p(\varepsilon) / 2})+B_{H^{-}}(\theta, \sqrt{p(\varepsilon) / 2})\right) \rightarrow U
$$

such that

$$
J(\lambda, \phi(\lambda, x+y))=\|x\|^{2}-\|y\|^{2} \quad \text { and } \quad \phi(\lambda, x+y)=\left(\lambda, \phi_{\lambda}(x+y)\right) \in \Lambda \times H
$$

for all $(\lambda, x, y) \in \Lambda \times B_{H^{+}}(\theta, \sqrt{p(\varepsilon) / 2}) \times B_{H^{-}}(\theta, \sqrt{p(\varepsilon) / 2})$. Moreover, for each $\lambda \in \Lambda, \phi_{\lambda}(0)=0, \phi_{\lambda}(x+y) \in H^{-}$if and only if $x=0$, and $\phi$ is a homoeomorphism from $\Lambda \times B_{H^{-}}(\theta, \sqrt{p(\varepsilon) / 2})$ onto $U \cap\left(\Lambda \times H^{-}\right)$according to any topology on both induced by any norm on $H^{-}$.

2.1. Proofs of Theorems 1.1, 1.3. Unlike the proof of $[13$, Theorem 2.1] and [14], we cannot directly apply Theorem 2.1 to the function $F^{\infty}$ in $(2.10)$ because $\bar{B}_{H_{\infty}^{0}}\left(\infty, R_{1}\right)$ is only locally compact. We must directly prove corresponding conclusions with those in Steps 1, 6, 7 of the proof of it given in Appendix A of [13], [14]. Moreover, in some steps we may prove the same parts of Theorem 1.1 and Theorem 1.3 in a unite way, in other steps we must deal with those two cases, respectively.

The following Lemma 2.2 (resp. Lemma 2.3) is the analogue of [13, Lemma 2.13] or [14, Lemma 3.1] under the condition $\left(\mathrm{E}_{\infty}\right)\left(\operatorname{resp} .\left(\mathrm{E}_{\infty}^{\prime}\right)\right)$.

LEMma 2.2. Under the above assumptions $(\mathrm{S}),\left(\mathrm{F} 1_{\infty}\right)-\left(\mathrm{F} 3_{\infty}\right)$ and $\left(\mathrm{C} 1_{\infty}\right)-$ $\left(\mathrm{C} 2_{\infty}\right)$, and $\left(\mathrm{E}_{\infty}\right)$ there exists a unique map $h^{\infty}: \bar{B}_{H_{\infty}^{0}}\left(\infty, R_{1}\right) \rightarrow \bar{B}_{X_{\infty}^{ \pm}}\left(\theta, \rho_{A}\right)$ (by increasing $R_{1}>0$ if necessary), which is Lipschitz continuous, such that

(a) $\left(I-P_{\infty}^{0}\right) A\left(z+h^{\infty}(z)\right)=\theta$ for all $z \in \bar{B}_{H_{\infty}^{0}}\left(\infty, R_{1}\right)$;

(b) $h^{\infty}$ is strictly F-differentiable at infinity and $d h^{\infty}(\infty)=0$ under the assumption $\left(\mathrm{SE}_{\infty}\right)$;

(c) $\lim _{\|z\|_{X} \rightarrow \infty}\left\|h^{\infty}(z)\right\|_{X}=0$ provided that $M(A)=0$ in the assumption $\left(\mathrm{E}_{\infty}\right)$;

(d) the function $\bar{B}_{H_{\infty}^{0}}\left(\infty, R_{1}\right) \rightarrow \mathbb{R}, z \mapsto \mathcal{L}^{\infty}(z):=\mathcal{L}\left(z+h^{\infty}(z)\right)$ is $C^{1}$ and $d \mathcal{L}^{\infty}\left(z_{0}\right)(z)=\left(A\left(z_{0}+h^{\infty}\left(z_{0}\right)\right), z\right)_{H} \quad$ for all $z_{0} \in \bar{B}_{H_{\infty}^{0}}\left(\infty, R_{1}\right), z \in H_{\infty}^{0} ;$

(e) If $P_{\infty}^{0} \circ A: X \rightarrow X_{\infty}^{0}$ has a strict Fréchet derivative $S \in L\left(X, X_{\infty}^{0}\right)$ at infinity, (for instance this is true when $A$ is strictly $F$-differentiable at infinity), then the function $\mathcal{L}^{\infty}$ is $C^{2-0}$, and $d \mathcal{L}^{\infty}$ has a strict Fréchet derivative zero provided $S=\left.P_{\infty}^{0} \circ B(\infty)\right|_{X}$;

(f) If $A$ is $C^{1}$ the maps $h^{\infty}$ and $\mathcal{L}^{\infty}$ are $C^{1}$ and $C^{2}$, respectively, and $d h^{\infty}(z)=-\left.\left[\left.\left(I-P_{\infty}^{0}\right) A^{\prime}\left(z+h^{\infty}(z)\right)\right|_{X_{\infty}^{ \pm}}\right]^{-1}\left(I-P_{\infty}^{0}\right) A^{\prime}\left(z+h^{\infty}(z)\right)\right|_{H_{\infty}^{0}} ;$

(g) If $\mathcal{L}$ is $C^{2}$ then $h^{\infty}$ is also $C^{1}$ as a map to $H_{\infty}^{ \pm}$(hence $X_{\infty}^{ \pm}$). 
Proof. (a) Consider the map $S^{\infty}: \bar{B}_{H_{\infty}^{0}}\left(\infty, R_{1}\right) \times \bar{B}_{X_{\infty}^{ \pm}}\left(\theta, \rho_{A}\right) \rightarrow X_{\infty}^{ \pm}$,

$$
(z, x) \mapsto-\left(\left.B(\infty)\right|_{X_{\infty}^{ \pm}}\right)^{-1}\left(I-P_{\infty}^{0}\right) A(z+x)+x .
$$

Let $z_{1}, z_{2} \in \bar{B}_{H_{\infty}^{0}}\left(\infty, R_{1}\right)$, and let $x_{1}, x_{2} \in \bar{B}_{X_{\infty}^{ \pm}}\left(\theta, \rho_{A}\right)$. Noting that $B(\infty) x_{i} \in$ $X_{\infty}^{ \pm}$and $B(\infty) z_{i}=0, i=1,2$, it follows from (1.2) that

$$
\begin{array}{r}
\left\|S^{\infty}\left(z_{1}, x_{1}\right)-S^{\infty}\left(z_{2}, x_{2}\right)\right\|_{X_{\infty}^{ \pm}} \leq C_{1}^{\infty} \cdot \|\left(I-P_{\infty}^{0}\right) A\left(z_{1}+x_{1}\right)-B(\infty) x_{1} \\
\quad-\left(I-P_{\infty}^{0}\right) A\left(z_{2}+x_{2}\right)+B(\infty) x_{2}\left\|_{X_{\infty}^{ \pm}} \leq \frac{1}{\kappa}\right\| z_{1}+x_{1}-z_{2}-x_{2} \|_{X} .
\end{array}
$$

In particular, for any $z \in \bar{B}_{H_{\infty}^{0}}\left(\infty, R_{1}\right)$ and $x_{1}, x_{2} \in \bar{B}_{X_{\infty}^{ \pm}}\left(\theta, \rho_{A}\right)$, we get

$$
\left\|S^{\infty}\left(z, x_{1}\right)-S^{\infty}\left(z, x_{2}\right)\right\|_{X_{\infty}^{ \pm}} \leq \frac{1}{\kappa}\left\|x_{1}-x_{2}\right\|_{X} .
$$

- If $\rho_{A}<\infty$ in $\left(\mathrm{E}_{\infty}\right)$, this means that

$$
M(A)<\infty \quad \text { and } \quad \rho_{A} \in\left(\frac{\kappa}{\kappa-1} C_{1}^{\infty} M(A), \infty\right) .
$$

By increasing $R_{1}>\rho_{A}$ we may derive

$$
\sup \left\{\left\|\left(I-P_{\infty}^{0}\right) A(z)\right\|_{X}: z \in H_{\infty}^{0},\|z\|_{X} \geq R_{1}\right\} \leq \frac{\kappa-1}{\kappa} \frac{\rho_{A}}{C_{1}^{\infty}}
$$

and hence

$$
\left\|S^{\infty}(z, \theta)\right\|_{X_{\infty}^{ \pm}} \leq\left\|\left(\left.B(\infty)\right|_{X_{\infty}^{ \pm}}\right)^{-1}\right\|_{L\left(X_{\infty}^{ \pm}\right)} \cdot\left\|\left(I-P_{\infty}^{0}\right) A(z)\right\|_{X_{\infty}^{ \pm}} \leq \frac{\kappa-1}{\kappa} \rho_{A} .
$$

It follows from this and (2.2) that

$$
\begin{aligned}
\left\|S^{\infty}(z, x)\right\|_{X_{\infty}^{ \pm}} & \leq\left\|S^{\infty}(z, x)-S^{\infty}(z, \theta)\right\|_{X_{\infty}^{ \pm}}+\left\|S^{\infty}(z, \theta)\right\|_{X_{\infty}^{ \pm}} \\
& \leq \frac{1}{\kappa}\|x\|_{X}+\frac{\kappa-1}{\kappa} \rho_{A} \leq \frac{1}{\kappa} \rho_{A}+\frac{\kappa-1}{\kappa} \rho_{A} \leq \rho_{A}
\end{aligned}
$$

for any $z \in \bar{B}_{H_{\infty}^{0}}\left(\infty, R_{1}\right)$ and $x \in \bar{B}_{X_{\infty}^{ \pm}}\left(\theta, \rho_{A}\right)$. Hence the Banach fixed point theorem gives a unique map $h^{\infty}: \bar{B}_{H_{\infty}^{0}}\left(\infty, R_{1}\right) \rightarrow \bar{B}_{X_{\infty}^{ \pm}}\left(\theta, \rho_{A}\right)$, which is also continuous, such that $S^{\infty}\left(z, h^{\infty}(z)\right)=h^{\infty}(z)$ or equivalently

$$
\left(I-P_{\infty}^{0}\right) A\left(z+h^{\infty}(z)\right)=\theta \quad \text { for all } z \in \bar{B}_{H_{\infty}^{0}}\left(\infty, R_{1}\right) .
$$

This and (2.1) imply

$$
\begin{aligned}
\left\|h^{\infty}\left(z_{1}\right)-h^{\infty}\left(z_{2}\right)\right\|_{X} & =\left\|S^{\infty}\left(z_{1}, h^{\infty}\left(z_{1}\right)\right)-S^{\infty}\left(z_{2}, h^{\infty}\left(z_{2}\right)\right)\right\|_{X_{\infty}^{ \pm}} \\
& \leq \frac{1}{\kappa}\left\|z_{1}+h^{\infty}\left(z_{1}\right)-z_{2}-h^{\infty}\left(z_{2}\right)\right\|_{X}
\end{aligned}
$$

and hence

(2.5) $\left\|h^{\infty}\left(z_{1}\right)-h^{\infty}\left(z_{2}\right)\right\|_{X} \leq \frac{1}{\kappa-1}\left\|z_{1}-z_{2}\right\|_{X} \quad$ for all $z_{1}, z_{2} \in \bar{B}_{H_{\infty}^{0}}\left(\infty, R_{1}\right)$.

That is, $h^{\infty}$ is Lipschitz continuous. 
- If $\rho_{A}=\infty$ in $\left(\mathrm{E}_{\infty}\right)$, then (2.1) holds for any $z \in \bar{B}_{H_{\infty}^{0}}\left(\infty, R_{1}\right)$ and $x_{1}, x_{2} \in$ $X_{\infty}^{ \pm}$. The Banach fixed point theorem gives a unique map $h^{\infty}: \bar{B}_{H_{\infty}^{0}}\left(\infty, R_{1}\right) \rightarrow$ $X_{\infty}^{ \pm}$, which is continuous, such that (2.4) and (2.5) also hold.

(b) If $M(A)<\infty$ in $\left(\mathrm{SE}_{\infty}\right)$ we choose $\kappa>1$ so large that $\rho_{A}>\frac{\kappa}{\kappa-1} C_{1}^{\infty} M(A)$. Then (1.2) is satisfied by increasing $R_{1}>0$ (if necessary). Hence (2.1)-(2.5) are still effective for these $\kappa$ and $R_{1}$. For $z_{i} \in \bar{B}_{H_{\infty}^{0}}\left(\theta, R_{1}\right)$ set $x_{i}=h^{\infty}\left(z_{i}\right)$ in (2.1), $i=1,2$. We obtain

$$
\begin{gathered}
\left\|h^{\infty}\left(z_{1}\right)-h^{\infty}\left(z_{2}\right)\right\|_{X_{\infty}^{ \pm}}=\left\|S^{\infty}\left(z_{1}, h^{\infty}\left(z_{1}\right)\right)-S^{\infty}\left(z_{2}, h^{\infty}\left(z_{2}\right)\right)\right\|_{X_{\infty}^{ \pm}} \\
\leq C_{1}^{\infty} \cdot \|\left(I-P_{\infty}^{0}\right) A\left(z_{1}+h^{\infty}\left(z_{1}\right)\right)-B(\infty)\left(z_{1}+h^{\infty}\left(z_{1}\right)\right) \\
-\left(I-P_{\infty}^{0}\right) A\left(h^{\infty}\left(z_{2}\right)\right)+B(\infty)\left(z_{2}+h^{\infty}\left(z_{2}\right)\right) \|_{X} .
\end{gathered}
$$

For any given small $\varepsilon>0$, since

$$
\left\|z_{i}+h^{\infty}\left(z_{i}\right)\right\|_{X}^{2} \geq\left\|z_{i}+h^{\infty}\left(z_{i}\right)\right\|^{2}=\left\|z_{i}\right\|^{2}+\left\|h^{\infty}\left(z_{i}\right)\right\|^{2} \geq\left\|z_{i}\right\|^{2},
$$

and $\left\|z_{i}\right\| \rightarrow \infty$ if and only if $\left\|z_{i}\right\|_{X} \rightarrow \infty$ for $z_{i} \in H_{\infty}^{0}, i=1,2$, by $\left(\mathrm{SE}_{\infty}\right)$ there exists $R>R_{1}$ such that for any $z_{i} \in \bar{B}_{H_{\infty}^{0}}(\infty, R), i=1,2$ we have

$$
\begin{aligned}
& \|\left(I-P_{\infty}^{0}\right) A\left(z_{1}+h^{\infty}\left(z_{1}\right)\right)-B(\infty)\left(z_{1}+h^{\infty}\left(z_{1}\right)\right) \\
& -\left(I-P_{\infty}^{0}\right) A\left(z_{2}+h^{\infty}\left(z_{2}\right)\right)+B(\infty)\left(z_{2}+h^{\infty}\left(z_{2}\right)\right) \|_{X} \\
& \quad \leq \varepsilon\left\|z_{1}+h^{\infty}\left(z_{1}\right)-z_{2}-h^{\infty}\left(z_{2}\right)\right\|_{X} \leq \frac{\kappa}{\kappa-1} \varepsilon\left\|z_{1}-z_{2}\right\|_{X}
\end{aligned}
$$

by (2.5). From this and (2.6) we derive that

$$
\left\|h^{\infty}\left(z_{2}\right)-h^{\infty}\left(z_{1}\right)\right\|_{X} \leq \frac{\kappa}{\kappa-1} C_{1}^{\infty} \varepsilon\left\|z_{2}-z_{1}\right\|_{X}
$$

for any $z_{i} \in \bar{B}_{H_{\infty}^{0}}(\infty, R), i=1,2$. This shows that $h^{\infty}$ has the strict Fréchet derivative zero at $\infty$.

(c) Recall that $h^{\infty}(z)$ is a unique fixed point in $\bar{B}_{X_{\infty}^{ \pm}}\left(\theta, \rho_{A}\right)$ of the map

$$
x \mapsto S^{\infty}(z, x)=-\left(\left.B(\infty)\right|_{X_{\infty}^{ \pm}}\right)^{-1}\left(I-P_{\infty}^{0}\right)[A(z+x)-B(\infty) x] .
$$

Since $M(A)=0$, for any small $0<\epsilon<\rho_{A}$ there exists a large $R>R_{1}$ such that

$$
\left\|\left(I-P_{\infty}^{0}\right) A(z)\right\|_{X^{ \pm}}<\frac{(\kappa-1) \varepsilon}{C_{1}^{\infty} \kappa}
$$

for any $z \in \bar{B}_{H_{\infty}^{0}}(\infty, R)$. By the deduction of (2.3), for any $z \in \bar{B}_{H_{\infty}^{0}}(\infty, R)$ and $x \in \bar{B}_{X_{\infty}^{ \pm}}(\theta, \epsilon)$ we have

$$
\begin{aligned}
\left\|S^{\infty}(z, x)\right\|_{X^{ \pm}} & \leq \frac{1}{\kappa}\|x\|_{X}+\left\|\left(\left.B(\infty)\right|_{X_{\infty}^{ \pm}}\right)^{-1}\left(I-P_{\infty}^{0}\right) A(z)\right\|_{X^{ \pm}} \\
& \leq \frac{1}{\kappa}\|x\|_{X}+C_{1}^{\infty}\left\|\left(I-P_{\infty}^{0}\right) A(z)\right\|_{X_{\infty}^{ \pm}} \leq \frac{\varepsilon}{\kappa}+\frac{(\kappa-1) \varepsilon}{\kappa}<\varepsilon .
\end{aligned}
$$


So the map $\bar{B}_{X_{\infty}^{ \pm}}(\theta, \varepsilon) \rightarrow \bar{B}_{X_{\infty}^{ \pm}}(\theta, \varepsilon), x \mapsto S^{\infty}(z, x)$ has a unique fixed point, which is, of course, contained in $\bar{B}_{X_{\infty}^{ \pm}}\left(\theta, \rho_{A}\right)$ and hence must be $h^{\infty}(z)$. This shows $\left\|h^{\infty}(z)\right\|_{X} \leq \varepsilon$.

(d) The proof is similar to Step 2 of proof of [13, Lemma 2.13] or [14, Lemma 3.1]. For any $z_{0} \in \bar{B}_{H_{\infty}^{0}}\left(\infty, R_{1}\right), z \in H_{\infty}^{0}$ and $t \in \mathbb{R} \backslash\{0\}$ with $z_{0}+t z \in \bar{B}_{H_{\infty}^{0}}\left(\infty, R_{1}\right)$, by the mean value theorem we have $s \in(0,1)$ such that

$$
\begin{aligned}
\mathcal{L}^{\infty}\left(z_{0}+t z\right) & -\mathcal{L}^{\infty}\left(z_{0}\right)=D \mathcal{L}\left(z_{s, t}\right)\left(t z+h^{\infty}\left(z_{0}+t z\right)-h^{\infty}\left(z_{0}\right)\right) \\
= & \left(A\left(z_{s, t}\right), t z+h^{\infty}\left(z_{0}+t z\right)-h^{\infty}\left(z_{0}\right)\right)_{H} \\
= & \left(A\left(z_{s, t}\right), t z\right)_{H}+\left(\left(I-P_{\infty}^{0}\right) A\left(z_{s, t}\right), h^{\infty}\left(z_{0}+t z\right)-h^{\infty}\left(z_{0}\right)\right)_{H}
\end{aligned}
$$

because $h^{\infty}\left(z_{0}+t z\right)-h^{\infty}\left(z_{0}\right) \in X_{\infty}^{ \pm} \subset H_{\infty}^{ \pm}$, where $z_{s, t}=z_{0}+h^{\infty}\left(z_{0}\right)+s[t z+$ $\left.h^{\infty}\left(z_{0}+t z\right)-h^{\infty}\left(z_{0}\right)\right]$. Note that $(2.5)$ implies

$$
\left\|h^{\infty}\left(z_{0}+t z\right)-h^{\infty}\left(z_{0}\right)\right\|_{H} \leq\left\|h^{\infty}\left(z_{0}+t z\right)-h^{\infty}\left(z_{0}\right)\right\|_{X} \leq \frac{1}{\kappa-1}|t| \cdot\|z\|_{X} .
$$

Let $t \rightarrow 0$, we have

$$
\begin{aligned}
& \left|\frac{\left(\left(I-P_{\infty}^{0}\right) A\left(z_{s, t}\right), h^{\infty}\left(z_{0}+t z\right)-h^{\infty}\left(z_{0}\right)\right)_{H}}{t}\right| \\
& \quad \leq \frac{\left\|\left(I-P_{\infty}^{0}\right) A\left(z_{s, t}\right)\right\|_{H} \cdot\left\|h^{\infty}\left(z_{0}+t z\right)-h^{\infty}\left(z_{0}\right)\right\|_{H}}{|t|} \\
& \quad \leq \frac{1}{\kappa-1}\|z\|_{X}\left\|\left(I-P_{\infty}^{0}\right) A\left(z_{s, t}\right)\right\|_{X} \\
& \quad \rightarrow \frac{1}{\kappa-1}\|z\|_{X} \cdot\left\|\left(I-P_{\infty}^{0}\right) A\left(z_{0}+h^{\infty}\left(z_{0}\right)\right)\right\|_{X}=0
\end{aligned}
$$

because of (2.4). From this and (2.8) it follows that

$$
D \mathcal{L}^{\infty}\left(z_{0}\right)(z)=\lim _{t \rightarrow 0} \frac{\mathcal{L}^{\infty}\left(z_{0}+t z\right)-\mathcal{L}^{\infty}\left(z_{0}\right)}{t}=\left(A\left(z_{0}+h^{\infty}\left(z_{0}\right)\right), z\right)_{H} .
$$

That is, $\mathcal{L}^{\infty}$ is Gâteaux differentiable at $z_{0}$. Clearly, $z \mapsto D \mathcal{L}^{\infty}\left(z_{0}\right)(z)$ is linear and continuous, i.e. $\mathcal{L}^{\infty}$ has a linear bounded Gâteaux derivative at $z_{0}$, $D \mathcal{L}^{\infty}\left(z_{0}\right)$, given by

$$
D \mathcal{L}^{\infty}\left(z_{0}\right) z=\left(A\left(z_{0}+h^{\infty}\left(z_{0}\right)\right), z\right)_{H}=\left(P_{\infty}^{0} A\left(z_{0}+h\left(z_{0}\right)\right), z\right)_{H} \quad \text { for all } z \in H_{\infty}^{0} .
$$

Note that $\left.B(\infty)\right|_{H_{\infty}^{0}}=0, B(\infty)\left(H_{\infty}^{ \pm}\right) \subset H_{\infty}^{ \pm}$and $h^{\infty}\left(z_{0}\right), h^{\infty}\left(z_{0}^{\prime}\right) \in X_{\infty}^{ \pm} \subset H_{\infty}^{ \pm}$ for any $z_{0}, z_{0}^{\prime} \in \bar{B}_{H_{\infty}^{0}}\left(\infty, R_{1}\right)$. We have

$$
\left(P_{\infty}^{0} B(\infty)\left(z_{0}+h^{\infty}\left(z_{0}\right)\right), z\right)_{H}=\left(P_{\infty}^{0} B(\infty)\left(z_{0}^{\prime}+h^{\infty}\left(z_{0}^{\prime}\right)\right), z\right)_{H}=0
$$

for all $z \in H_{\infty}^{0}$. From this it easily follows that

$$
\begin{aligned}
\left|D \mathcal{L}^{\infty}\left(z_{0}\right) z-D \mathcal{L}^{\infty}\left(z_{0}^{\prime}\right) z\right|=\left|\left(P_{\infty}^{0} A\left(z_{0}+h^{\infty}\left(z_{0}\right)\right)-P_{\infty}^{0} A\left(z_{0}^{\prime}+h^{\infty}\left(z_{0}^{\prime}\right)\right), z\right)_{H}\right| \\
=\mid\left(P_{\infty}^{0} A\left(z_{0}+h^{\infty}\left(z_{0}\right)\right)-P_{\infty}^{0} B(\infty)\left(z_{0}+h^{\infty}\left(z_{0}\right)\right), z\right)_{H} \\
\quad-\left(P_{\infty}^{0} A\left(z_{0}^{\prime}+h^{\infty}\left(z_{0}^{\prime}\right)\right)-P_{\infty}^{0} B(\infty)\left(z_{0}^{\prime}+h^{\infty}\left(z_{0}^{\prime}\right)\right), z\right)_{H} \mid
\end{aligned}
$$




$$
\begin{aligned}
\leq & \| P_{\infty}^{0} A\left(z_{0}+h^{\infty}\left(z_{0}\right)\right)-P_{\infty}^{0} B(\infty)\left(z_{0}+h^{\infty}\left(z_{0}\right)\right) \\
& -P_{\infty}^{0} A\left(z_{0}^{\prime}+h^{\infty}\left(z_{0}^{\prime}\right)\right)+P_{\infty}^{0} B(\infty)\left(z_{0}^{\prime}+h^{\infty}\left(z_{0}^{\prime}\right)\right)\left\|_{H} \cdot\right\| z \|_{H} \\
\leq & {\left[\left\|A\left(z_{0}+h^{\infty}\left(z_{0}\right)\right)-A\left(z_{0}^{\prime}+h^{\infty}\left(z_{0}^{\prime}\right)\right)\right\|_{X}\right.} \\
& \left.+\left\|B(\infty)\left(z_{0}+h^{\infty}\left(z_{0}\right)\right)-B(\infty)\left(z_{0}^{\prime}+h^{\infty}\left(z_{0}^{\prime}\right)\right)\right\|_{H}\right] \cdot\|z\|_{X}
\end{aligned}
$$

and hence

$$
\begin{aligned}
\left\|D \mathcal{L}^{\infty}\left(z_{0}\right)-D \mathcal{L}^{\infty}\left(z_{0}^{\prime}\right)\right\|_{\left(X_{\infty}^{0}\right)^{*}} & \leq\left\|A\left(z_{0}+h^{\infty}\left(z_{0}\right)\right)-A\left(z_{0}^{\prime}+h^{\infty}\left(z_{0}^{\prime}\right)\right)\right\|_{X} \\
+ & \left\|B(\infty)\left(z_{0}+h^{\infty}\left(z_{0}\right)\right)-B(\infty)\left(z_{0}^{\prime}+h^{\infty}\left(z_{0}^{\prime}\right)\right)\right\|_{H},
\end{aligned}
$$

where $\left(X_{\infty}^{0}\right)^{*}=\left(H_{\infty}^{0}\right)^{*}=L\left(X_{\infty}^{0}, \mathbb{R}\right)$.

Since both $A: X \rightarrow X$ and $B(\infty): H \rightarrow H$ are continuous by $\left(\mathrm{F} 2_{\infty}\right.$ ), from (2.5) we derive that $z_{0} \mapsto D \mathcal{L}^{\infty}\left(z_{0}\right)$ is continuous and therefore that $\mathcal{L}^{\infty}$ is Fréchet differentiable at $z_{0}$ and its Fréchet differential $d \mathcal{L}^{\infty}\left(z_{0}\right)=D \mathcal{L}^{\infty}\left(z_{0}\right)$. Moreover, the above estimate also shows that $z_{0} \mapsto d \mathcal{L}^{\infty}\left(z_{0}\right)$ is continuous.

(e) Since $P_{\infty}^{0} \circ A$ has the strict Fréchet derivative $S \in L\left(X, X_{\infty}^{0}\right)$ at $\infty$ then

$$
\left\|P_{\infty}^{0} \circ A\left(x_{1}\right)-P_{\infty}^{0} \circ A\left(x_{2}\right)-S\left(x_{1}-x_{2}\right)\right\|_{X} \leq \widehat{K}_{R}\left\|x_{1}-x_{2}\right\|_{X}
$$

for all $x_{1}, x_{2} \in B_{X}(\infty, R)$ with constant $\widehat{K}_{R} \rightarrow 0$ as $R \rightarrow \infty$.

Let $C>0$ be such that $\|z\|_{X} \leq C\|z\|$ for all $z \in H_{\infty}^{0}$. For $R>R_{1}$ and any $z_{0}, z_{0}^{\prime} \in B_{H_{\infty}^{0}}(\infty, R)$, since

$$
\left\|z+h^{\infty}(z)\right\|_{X}^{2} \geq\left\|z+h^{\infty}(z)\right\|^{2}=\|z\|^{2}+\left\|h^{\infty}(z)\right\|^{2} \geq\|z\|^{2} \quad \text { for } z=z_{0}, z_{0}^{\prime},
$$

it follows from the proof of (d), (2.9) and (2.5) that

$$
\begin{aligned}
\mid d \mathcal{L}^{\infty}\left(z_{0}\right) z & -d \mathcal{L}^{\infty}\left(z_{0}^{\prime}\right) z-\left(S\left(z_{0}+h^{\infty}\left(z_{0}\right)-z_{0}^{\prime}-h^{\infty}\left(z_{0}^{\prime}\right)\right), z\right)_{H} \mid \\
= & \mid\left(P_{\infty}^{0} A\left(z_{0}+h^{\infty}\left(z_{0}\right)\right)-P_{\infty}^{0} A\left(z_{0}^{\prime}+h^{\infty}\left(z_{0}^{\prime}\right)\right), z\right)_{H} \\
& -\left(S\left(z_{0}+h^{\infty}\left(z_{0}\right)-z_{0}^{\prime}-h^{\infty}\left(z_{0}^{\prime}\right)\right), z\right)_{H} \mid \\
\leq & \| P_{\infty}^{0} A\left(z_{0}+h^{\infty}\left(z_{0}\right)\right)-P_{\infty}^{0} A\left(z_{0}^{\prime}+h^{\infty}\left(z_{0}^{\prime}\right)\right) \\
& -S\left(z_{0}+h^{\infty}\left(z_{0}\right)-z_{0}^{\prime}-h^{\infty}\left(z_{0}^{\prime}\right)\right)\left\|_{H} \cdot\right\| z \|_{H} \\
\leq & \| P_{\infty}^{0} A\left(z_{0}+h^{\infty}\left(z_{0}\right)\right)-P_{\infty}^{0} A\left(z_{0}^{\prime}+h^{\infty}\left(z_{0}^{\prime}\right)\right) \\
& -S\left(z_{0}+h^{\infty}\left(z_{0}\right)-z_{0}^{\prime}-h^{\infty}\left(z_{0}^{\prime}\right)\right)\left\|_{X} \cdot\right\| z \|_{X} \\
\leq & \widehat{K}_{R} \cdot\left\|z_{0}+h^{\infty}\left(z_{0}\right)-z_{0}^{\prime}-h^{\infty}\left(z_{0}^{\prime}\right)\right\|_{X} \cdot\|z\|_{X} \\
\leq & \frac{\kappa}{\kappa-1} \widehat{K}_{R} \cdot\left\|z_{0}-z_{0}^{\prime}\right\|_{X} \cdot\|z\|_{X} \leq \frac{\kappa}{\kappa-1} C^{2} \widehat{K}_{R} \cdot\left\|z_{0}-z_{0}^{\prime}\right\|_{X} \cdot\|z\|
\end{aligned}
$$

for any $z \in H_{\infty}^{0}$. Hence

$$
\begin{aligned}
\left\|d \mathcal{L}^{\infty}\left(z_{0}\right)-d \mathcal{L}^{\infty}\left(z_{0}^{\prime}\right)\right\|_{L\left(H_{\infty}^{0}, \mathbb{R}\right)} & \\
\leq & \frac{\kappa}{\kappa-1} C^{2} \widehat{K}_{R} \cdot\left\|z_{0}-z_{0}^{\prime}\right\|_{X}+\left\|S\left(z_{0}+h^{\infty}\left(z_{0}\right)-z_{0}^{\prime}-h^{\infty}\left(z_{0}^{\prime}\right)\right)\right\|_{X} \\
\leq & \frac{\kappa}{\kappa-1}\left(1+C^{2} \widehat{K}_{R}\right) \cdot\left\|z_{0}-z_{0}^{\prime}\right\|_{X},
\end{aligned}
$$


that is, $\mathcal{L}^{\infty}$ is $C^{2-0}$. Moreover, if $S=\left.P_{\infty}^{0} \circ B(\infty)\right|_{X}$, then $\left(S\left(z_{0}+h^{\infty}\left(z_{0}\right)-z_{0}^{\prime}-\right.\right.$ $\left.\left.h^{\infty}\left(z_{0}^{\prime}\right)\right), z\right)_{H}=0$ for all $z \in H_{\infty}^{0}$, and hence

$$
\begin{aligned}
\mid d \mathcal{L}^{\infty}\left(z_{0}\right) & z-d \mathcal{L}^{\infty}\left(z_{0}^{\prime}\right) z \| \\
& =\left|d \mathcal{L}^{\infty}\left(z_{0}\right) z-d \mathcal{L}^{\infty}\left(z_{0}^{\prime}\right) z-\left(S\left(z_{0}+h^{\infty}\left(z_{0}\right)-z_{0}^{\prime}-h^{\infty}\left(z_{0}^{\prime}\right)\right), z\right)_{H}\right| \\
& \leq \frac{\kappa}{\kappa-1} C^{2} \widehat{K}_{R} \cdot\left\|z_{0}-z_{0}^{\prime}\right\|_{X} \cdot\|z\|
\end{aligned}
$$

for any $z \in H_{\infty}^{0}$. This implies

$$
\frac{\left\|d \mathcal{L}^{\infty}\left(z_{0}\right)-d \mathcal{L}^{\infty}\left(z_{0}^{\prime}\right)\right\|_{L\left(H_{\infty}^{0}, \mathbb{R}\right)}}{\left\|z_{0}-z_{0}^{\prime}\right\|} \rightarrow 0
$$

as $\left(\left\|z_{0}\right\|,\left\|z_{0}^{\prime}\right\|\right) \rightarrow(\infty, \infty)$ and $z_{0} \neq z_{0}^{\prime}$. Hence $d \mathcal{L}^{\infty}$ has the strict Fréchet derivative zero at infinity.

(f) Since $A$ is $C^{1}$ the corresponding conclusions can be obtained as in [13, Remark 2.14] or [14, Remark 3.2].

(g) If $\mathcal{L}$ is $C^{2}$ then $\nabla \mathcal{L}(x)=A(x)$ for all $x \in X_{\infty}$. For $z_{0} \in \bar{B}_{H_{\infty}^{0}}\left(\infty, R_{1}\right)$ we have $\left(I-P_{\infty}^{0}\right) \nabla \mathcal{L}\left(z_{0}+h^{\infty}\left(z_{0}\right)\right)=\theta$. By the implicit function theorem there exists a neighbourhood $\mathcal{O}\left(z_{0}\right)$ of $z_{0}$ in $\bar{B}_{H_{\infty}^{0}}\left(\infty, R_{1}\right)$ and a unique $C^{1}$ map $h: \mathcal{O}\left(z_{0}\right) \rightarrow H_{\infty}^{ \pm}$such that $\left(I-P_{\infty}^{0}\right) \nabla \mathcal{L}(z+h(z))=\theta$ for all $z \in \mathcal{O}\left(z_{0}\right)$. Moreover,

$$
\left(I-P_{\infty}^{0}\right) \nabla \mathcal{L}\left(z+h^{\infty}(z)\right)=\left(I-P_{\infty}^{0}\right) A\left(z+h^{\infty}(z)\right)=\theta
$$

for all $z \in \bar{B}_{H_{\infty}^{0}}\left(\infty, R_{1}\right)$, and $h^{\infty}$ is also continuous as a map to $H_{\infty}^{ \pm}$, by the implicit function theorem (precisely its proof) we get $h(z)=h^{\infty}(z)$ for all $z \in$ $\mathcal{O}\left(z_{0}\right)$. The desired conclusion is proved.

LEMma 2.3. Under the above assumptions $(\mathrm{S}),\left(\mathrm{F} 1_{\infty}\right)-\left(\mathrm{F} 3_{\infty}\right)$ and $\left(\mathrm{C} 1_{\infty}\right)-$ $\left(\mathrm{C} 2_{\infty}\right)$, and $\left(\mathrm{E}_{\infty}^{\prime}\right)$ there exist $R_{1}>0$ and a unique map

$$
h^{\infty}: \bar{B}_{H_{\infty}^{0}}\left(\infty, R_{1}\right) \rightarrow \bar{B}_{X}\left(\theta, \rho_{A}\right) \cap X_{\infty}^{ \pm},
$$

which is continuous, such that

(a) $\left(I-P_{\infty}^{0}\right) A\left(z+h^{\infty}(z)\right)=\theta$ for all $z \in \bar{B}_{H_{\infty}^{0}}\left(\infty, R_{1}\right)$;

(b) $\lim _{\|z\|_{X} \rightarrow \infty}\left\|h^{\infty}(z)\right\|_{X}=0$ provided that $M(A)=0$ in $\left(\mathrm{E}_{\infty}^{\prime}\right)$;

(c) If $A$ is $C^{1}$, then $h^{\infty}$ is $C^{1}$ and

$$
d h^{\infty}(z)=-\left.\left[\left.\left(I-P_{\infty}^{0}\right) A^{\prime}\left(z+h^{\infty}(z)\right)\right|_{X_{\infty}^{ \pm}}\right]^{-1}\left(I-P_{\infty}^{0}\right) A^{\prime}\left(z+h^{\infty}(z)\right)\right|_{H_{\infty}^{0}} .
$$

Moreover, the functional $\mathcal{L}^{\infty}: \bar{B}_{H_{\infty}^{0}}\left(\infty, R_{1}\right) \rightarrow \mathbb{R}, z \mapsto \mathcal{L}\left(z+h^{\infty}(z)\right)$ is $C^{2}$ and $d \mathcal{L}^{\infty}\left(z_{0}\right)(z)=\left(A\left(z_{0}+h^{\infty}\left(z_{0}\right)\right), z\right)_{H}$ for all $z_{0} \in \bar{B}_{H_{\infty}^{0}}\left(\infty, R_{1}\right)$ and $z \in H_{\infty}^{0}$;

(d) If $\mathcal{L}$ is $C^{2}$ then $h^{\infty}$ is also $C^{1}$ as a map to $H_{\infty}^{ \pm}$(hence $X_{\infty}^{ \pm}$).

Proof. Recall the proof of Lemma 2.2(a). Under the condition $\left(\mathrm{E}_{\infty}^{\prime}\right)$, we can only obtain (1.4) and (2.1) for $z_{1}=z_{2}$. Hence (2.2) still holds. Unless (2.1) and (2.5) the proof of Lemma 2.2(a) is valid. 
The proof of (b) is the same as that of Lemma 2.2(c). (c)-(d) can be obtained by the implicit function theorem as usual.

Define a continuous map

$$
F^{\infty}: \bar{B}_{H_{\infty}^{0}}\left(\infty, R_{1}\right) \times H_{\infty}^{ \pm} \rightarrow \mathbb{R}
$$

by $F^{\infty}(z, u)=\mathcal{L}\left(z+h^{\infty}(z)+u\right)-\mathcal{L}\left(z+h^{\infty}(z)\right)$. Then for each $z \in \bar{B}_{H_{\infty}^{0}}\left(\infty, R_{1}\right)$ the map $F^{\infty}(z, \cdot)$ is continuously directional differentiable on $H_{\infty}^{ \pm}$, and the directional derivative of it at $u \in H_{\infty}^{ \pm}$in any direction $v \in H_{\infty}^{ \pm}$is given by

$$
D_{2} F^{\infty}(z, u)(v)=\left(A\left(z+h^{\infty}(z)+u\right), v\right)_{H}=\left(\left(I-P_{\infty}^{0}\right) A\left(z+h^{\infty}(z)+u\right), v\right)_{H} .
$$

It follows from this and (2.4) that

$$
F^{\infty}(z, \theta)=0 \quad \text { and } \quad D_{2} F^{\infty}(z, \theta)(v)=0 \quad \text { for all } v \in H_{\infty}^{ \pm} .
$$

Later on, if (1.5) holds we shall assume (by increasing $R_{1}>0$ ) that

$$
-\frac{a_{\infty}}{8}\|z+u\|^{2} \leq \mathcal{L}(z+u)-\frac{1}{2}(B(\infty) u, u)_{H} \leq \frac{a_{\infty}}{8}\|z+u\|^{2}
$$

for any $(z, u) \in \bar{B}_{H_{\infty}^{0}}\left(\infty, R_{1}\right) \times H_{\infty}^{ \pm}$.

Under the assumptions $\left(\mathrm{C} 1_{\infty}\right)-\left(\mathrm{C} 2_{\infty}\right)$ and $\left(\mathrm{D}_{\infty}\right)$, with the same proof methods we can obtain the corresponding results with [13, Lemma 2.15] and [13, Lemma 2.16] (or [14, Lemma 3.3] and [14, Lemma 3.4]) as follows.

LEMma 2.4. There exists a function $\omega_{\infty}: V_{\infty} \cap X \rightarrow[0, \infty)$ with the property that $\omega_{\infty}(x) \rightarrow 0$ as $x \in V_{\infty} \cap X$ and $\|x\| \rightarrow \infty$, such that

$$
\left|(B(x) u, v)_{H}-(B(\infty) u, v)_{H}\right| \leq \omega_{\infty}(x)\|u\| \cdot\|v\|
$$

for any $x \in V_{\infty} \cap X, u \in H_{\infty}^{0} \oplus H_{\infty}^{-}$and $v \in H$.

LEMMA 2.5. Let $a_{\infty}>0$ as in (1.1). By increasing $R_{1}$ we may find a number $a_{1} \in\left(0,2 a_{\infty}\right]$ such that for any $x \in \bar{B}_{H}\left(\infty, R_{1}\right) \cap X$ one has

(a) $(B(x) u, u)_{H} \geq a_{1}\|u\|^{2}$ for all $u \in H_{\infty}^{+}$;

(b) $\left|(B(x) u, v)_{H}\right| \leq \omega_{\infty}(x)\|u\| \cdot\|v\|$ for all $u \in H_{\infty}^{+}$and all $v \in H_{\infty}^{-} \oplus H_{\infty}^{0}$;

(c) $(B(x) u, u)_{H} \leq-a_{\infty}\|u\|^{2}$ for all $u \in H_{\infty}^{-}$.

Note: Actually, for the proof of Theorem 1.1 (resp. Theorem 1.3) we only need that Lemmas 2.4 and 2.5 hold in a set of form

$$
\left.\bar{B}_{H_{\infty}^{0}}\left(\infty, R^{\prime}\right) \oplus X_{\infty}^{ \pm} \quad \text { (resp. } \bar{B}_{H_{\infty}^{0}}\left(\infty, R^{\prime}\right) \oplus\left(\bar{B}_{H}\left(\theta, r^{\prime}\right) \cap X_{\infty}^{ \pm}\right)\right) .
$$

In this case we can only get the following Lemma 2.6 in such a set too.

As in the proof of [13, Lemma 2.17] or [14, Lemma 3.5] we can use the above lemmas to prove:

Lemma 2.6. The functional $F^{\infty}$ in (2.10) satisfies (a)-(d) in Theorem 2.1, i.e.

(a) $F^{\infty}(z, \theta)=0$ and $D_{2} F^{\infty}(z, \theta)=0$ for any $z \in \bar{B}_{H_{\infty}^{0}}\left(\infty, R_{1}\right)$; 
(b) $\left[D_{2} F^{\infty}\left(z, u+v_{2}\right)-D_{2} F^{\infty}\left(z, u+v_{1}\right)\right]\left(v_{2}-v_{1}\right) \leq-a_{\infty}\left\|v_{2}-v_{1}\right\|^{2}<0$ for any $(z, u) \in \bar{B}_{H_{\infty}^{0}}\left(\infty, R_{1}\right) \times H_{\infty}^{+}, v_{1}, v_{2} \in H_{\infty}^{-}$with $v_{1} \neq v_{2}$;

(c) $D_{2} F^{\infty}(z, u+v)(u-v) \geq a_{1}\|u\|^{2}+a_{\infty}\|v\|^{2}>0$ for any $(z, u, v) \in$ $\bar{B}_{H_{\infty}^{0}}\left(\infty, R_{1}\right) \times H_{\infty}^{+} \times H_{\infty}^{-}$with $(u, v) \neq(\theta, \theta)$;

(d) $D_{2} F^{\infty}(z, u) u \geq a_{1}\|u\|^{2}>p(\|u\|)$ for any $(z, u) \in \bar{B}_{H_{\infty}^{0}}\left(\infty, R_{1}\right) \times H_{\infty}^{+}$ with $u \neq \theta$, where $p(t)=a_{1} t^{2} / 2$.

Proof. By (2.11) it suffices to prove that $F^{\infty}$ satisfies conditions (b)-(d).

Step 1. For any $z \in \bar{B}_{H_{\infty}^{0}}\left(\infty, R_{1}\right), u^{+} \in X_{\infty}^{+}$and $u_{1}^{-}, u_{2}^{-} \in H_{\infty}^{-}$, as in the proof of [13, Lemma 2.17] or [14, Lemma 3.5], since the function

$$
u \mapsto\left(A\left(z+h^{\infty}(z)+u^{+}+u\right), u_{2}^{-}-u_{1}^{-}\right)_{H}
$$

is continuously directional differentiable, by the condition $\left(\mathrm{F} 2{ }_{\infty}\right)$ and the mean value theorem we have a number $t \in(0,1)$ such that

$$
\begin{aligned}
{\left[D_{2} F^{\infty}\right.} & \left.\left(z, u^{+}+u_{2}^{-}\right)-D_{2} F^{\infty}\left(z, u^{+}+u_{1}^{-}\right)\right]\left(u_{2}^{-}-u_{1}^{-}\right) \\
= & \left(A\left(z+h^{\infty}(z)+u^{+}+u_{2}^{-}\right), u_{2}^{-}-u_{1}^{-}\right)_{H} \\
& -\left(A\left(z+h^{\infty}(z)+u^{+}+u_{1}^{-}\right), u_{2}^{-}-u_{1}^{-}\right)_{H} \\
= & \left(D A\left(z+h^{\infty}(z)+u^{+}+u_{1}^{-}+t\left(u_{2}^{-}-u_{1}^{-}\right)\right)\left(u_{2}^{-}-u_{1}^{-}\right), u_{2}^{-}-u_{1}^{-}\right)_{H} \\
= & \left(B\left(z+h(z)+u^{+}+u_{1}^{-}+t\left(u_{2}^{-}-u_{1}^{-}\right)\right)\left(u_{2}^{-}-u_{1}^{-}\right), u_{2}^{-}-u_{1}^{-}\right)_{H} \\
\leq & -a_{\infty}\left\|u_{2}^{-}-u_{1}^{-}\right\|^{2},
\end{aligned}
$$

where the third equality comes from $\left(\mathrm{F} 3_{\infty}\right)$, and the final inequality is due to Lemma 2.5(c). Hence the density of $X_{\infty}^{+}$in $H_{\infty}^{+}$leads to

$$
\left[D_{2} F^{\infty}\left(z, u^{+}+u_{2}^{-}\right)-D_{2} F^{\infty}\left(z, u^{+}+u_{1}^{-}\right)\right]\left(u_{2}^{-}-u_{1}^{-}\right) \leq-a_{0}\left\|u_{2}^{-}-u_{1}^{-}\right\|^{2}
$$

for all $z \in \bar{B}_{H_{\infty}^{0}}\left(\infty, R_{1}\right), u^{+} \in H^{+}$and $u_{1}^{-}, u_{2}^{-} \in H^{-}$. This implies the condition (b).

Step 2. For $z \in \bar{B}_{H_{\infty}^{0}}\left(\infty, R_{1}\right), u^{+} \in X_{\infty}^{+}$and $u^{-} \in H_{\infty}^{-}$, using (2.11), the mean value theorem and $\left(\mathrm{F} 2_{\infty}\right)-\left(\mathrm{F} 3_{\infty}\right)$, for some $t \in(0,1)$ we have

$$
\begin{aligned}
D_{2} F^{\infty} & \left(z, u^{+}+u^{-}\right)\left(u^{+}-u^{-}\right) \\
= & D_{2} F^{\infty}\left(z, u^{+}+u^{-}\right)\left(u^{+}-u^{-}\right)-D_{2} F^{\infty}(z, \theta)\left(u^{+}-u^{-}\right) \\
= & \left(A\left(z+h^{\infty}(z)+u^{+}+u^{-}\right), u^{+}-u^{-}\right)_{H}-\left(A\left(z+h^{\infty}(z)+\theta\right), u^{+}-u^{-}\right)_{H} \\
= & \left(B\left(z+h^{\infty}(z)+t\left(u^{+}+u^{-}\right)\right)\left(u^{+}+u^{-}\right), u^{+}-u^{-}\right)_{H} \\
= & \left(B\left(z+h^{\infty}(z)+t\left(u^{+}+u^{-}\right)\right) u^{+}, u^{+}\right)_{H} \\
& -\left(B\left(z+h^{\infty}(z)+t\left(u^{+}+u^{-}\right)\right) u^{-}, u^{-}\right)_{H} \geq a_{1}\left\|u^{+}\right\|^{2}+a_{\infty}\left\|u^{-}\right\|^{2} .
\end{aligned}
$$

The final inequality comes from Lemma 2.5(a) and (c). The condition (c) follows because $X_{\infty}^{+}$is dense in $H_{\infty}^{+}$. 
Step 3. For $z \in \bar{B}_{H_{\infty}^{0}}\left(\infty, R_{1}\right)$ and $u^{+} \in X_{\infty}^{+}$, as above we may use the mean value theorem to get a number $t \in(0,1)$ such that

$$
\begin{aligned}
D_{2} F^{\infty}\left(z, u^{+}\right) u^{+} & =D_{2} F^{\infty}\left(z, u^{+}\right) u^{+}-D_{2} F^{\infty}(z, \theta) u^{+} \\
& =\left(A\left(z+h^{\infty}(z)+u^{+}\right), u^{+}\right)_{H}-\left(A\left(z+h^{\infty}(z)+\theta\right), u^{+}\right)_{H} \\
& =\left(B\left(z+h^{\infty}(z)+t u^{+}\right) u^{+}, u^{+}\right)_{H} \geq a_{1}\left\|u^{+}\right\|^{2} .
\end{aligned}
$$

The final inequality is because of Lemma 2.5(a). The condition (d) follows.

[Note: The condition $\nu_{\infty}>0$ is essentially used in the proofs of the above lemma. If $\nu_{\infty}=0$ the arguments before Lemma 2.4 is not needed. In this case Lemmas 2.4, 2.5 also hold with $H_{\infty}^{0}=\{\theta\}$. When replaceing $F^{\infty}$ with $\mathcal{L}$ the corresponding conclusions in Lemma 2.6 cannot be proved if no further conditions are imposed on $\mathcal{L}$. (See proof of Lemma 2.16).]

Now $\bar{B}_{H_{\infty}^{0}}\left(\infty, R_{1}\right)$ is only locally compact, we cannot directly apply Theorem 2.1 to the function $F^{\infty}$. Recall that the compactness are only used in Steps 1 and 6 of proof of $[13,14$, Theorem A.1]. (See the proof of more general [13, 14, Claim A.3]). We shall directly prove these two steps in the present case. To this end we need the following result.

Lemma 2.7. (a) Let $\left\{z_{k}\right\} \subset V_{\infty} \cap H_{\infty}^{0}$ and $\left\{u_{k}\right\} \subset H_{\infty}^{ \pm}$such that $\left\|z_{k}\right\| \rightarrow \infty$ and that $\left\|u_{k}-u_{0}\right\| \rightarrow 0$ for some $u_{0} \in H$. Then

$$
F^{\infty}\left(z_{k}, u_{k}\right) \rightarrow \frac{1}{2}\left(B(\infty) u_{0}, u_{0}\right)_{H} \quad \text { as } k \rightarrow \infty .
$$

(b) If $\mathcal{L}(u)=(B(\infty) u, u)_{H} / 2+o\left(\|u\|^{2}\right)$ as $\|u\| \rightarrow \infty$, then

$$
\begin{aligned}
\frac{a_{\infty}}{4} \| u^{+} & \left\|^{2}-2\right\| B(\infty)\|\cdot\| u^{-}\left\|^{2}-\frac{2\|B(\infty)\|^{2}}{a_{\infty}} \cdot\right\| h^{\infty}(z)\left\|^{2}-\frac{a_{\infty}}{2}\right\| z \|^{2} \\
& \leq F^{\infty}\left(z, u^{+}+u^{-}\right) \\
& \leq 2\|B(\infty)\| \cdot\left\|u^{+}\right\|^{2}-\frac{a_{\infty}}{4}\left\|u^{-}\right\|^{2}+\frac{a_{\infty}}{2}\|z\|^{2}+\frac{2\|B(\infty)\|^{2}}{a_{\infty}}\left\|h^{\infty}(z)\right\|^{2} .
\end{aligned}
$$

for any $\left(z, u^{+}, u^{-}\right) \in \bar{B}_{H_{\infty}^{0}}\left(\infty, R_{1}\right) \times H_{\infty}^{+} \times H_{\infty}^{-}$. Consequently, for any given $\left(z_{0}, u_{0}^{+}\right) \in \bar{B}_{H_{\infty}^{0}}\left(\infty, R_{1}\right) \times H_{\infty}^{+}$there exists a neighbourhood $\mathcal{U}$ of it in $\bar{B}_{H_{\infty}^{0}}\left(\infty, R_{1}\right)$ $\times H_{\infty}^{+}$such that $F^{\infty}\left(z, u^{+}+u^{-}\right) \rightarrow-\infty$ uniformly in $\left(z, u^{+}\right) \in \mathcal{U}$ as $u^{-} \in H_{\infty}^{-}$ and $\left\|u^{-}\right\| \rightarrow \infty$.

Proof. (a) Since $F^{\infty}$ is continuous and $X_{\infty}^{ \pm}$is dense in $H_{\infty}^{ \pm}$we can choose $\left\{u_{k}^{\prime}\right\} \subset X_{\infty}^{ \pm}$such that $\left\|u_{k}^{\prime}-u_{0}\right\| \rightarrow 0$ and $\left|F^{\infty}\left(z_{k}, u_{k}\right)-F^{\infty}\left(z_{k}, u_{k}^{\prime}\right)\right|<1 / k$ for $k=1,2, \ldots$ Hence we can assume that $\left\{u_{k}\right\} \subset X_{\infty}^{ \pm}$in the sequel without loss of generality.

Note that $h^{\infty}\left(z_{k}\right)+s t u_{k} \in X_{\infty}^{ \pm} \subset H_{\infty}^{ \pm}$and

$$
\left\|z_{k}+h^{\infty}\left(z_{k}\right)+s t u_{k}\right\|^{2}=\left\|z_{k}\right\|^{2}+\left\|h^{\infty}\left(z_{k}\right)+s t u_{k}\right\|^{2} \geq\left\|z_{k}\right\|^{2}
$$


for all $s, t \in[0,1]$ and $k=1,2, \ldots$ By $\left(\mathrm{D} 2_{\infty}\right)$, for any $u \in H$ we have

$$
\lim _{k \rightarrow \infty}\left\|P\left(z_{k}+h^{\infty}\left(z_{k}\right)+s t u_{k}\right) u-P(\infty) u\right\|=0
$$

uniformly in $s, t \in[0,1]$. Then the principle of uniform boundedness implies

$$
M(P):=\sup \left\{\left\|P\left(z_{k}+h^{\infty}\left(z_{k}\right)+s t u_{k}\right)\right\|_{L(H)} \mid k \in \mathbb{N}, s, t \in[0,1]\right\}<\infty
$$

Moreover, by $\left(\mathrm{D} 3_{\infty}\right)$ we have also

$$
\lim _{k \rightarrow \infty}\left\|Q\left(z_{k}+h^{\infty}\left(z_{k}\right)+s t u_{k}\right)-Q(\infty)\right\|_{L(H)}=0
$$

uniformly in $s, t \in[0,1]$. It follows from (2.13) and (2.14) that

$$
\begin{aligned}
\mid\left(P \left(z_{k}+h^{\infty}\left(z_{k}\right)\right.\right. & \left.\left.+s t u_{k}\right) u_{k}, u_{k}\right)_{H}-\left(P(\infty) u_{0}, u_{0}\right)_{H} \mid \\
= & \mid\left(P\left(z_{k}+h^{\infty}\left(z_{k}\right)+s t u_{k}\right)\left(u_{k}-u_{0}\right), u_{k}\right)_{H} \\
& +\left(P\left(z_{k}+h^{\infty}\left(z_{k}\right)+s t u_{k}\right) u_{0}, u_{k}-u_{0}\right)_{H} \\
& +\left(P\left(z_{k}+h^{\infty}\left(z_{k}\right)+s t u_{k}\right) u_{0}, u_{0}\right)_{H}-\left(P(\infty) u_{0}, u_{0}\right)_{H} \mid \\
\leq & \left\|P\left(z_{k}+h^{\infty}\left(z_{k}\right)+s t u_{k}\right)\right\|_{L(H)}\left\|u_{k}-u_{0}\right\| \cdot\left\|u_{k}\right\| \\
& +\left\|P\left(z_{k}+h^{\infty}\left(z_{k}\right)+s t u_{k}\right) u_{0}\right\| \cdot\left\|u_{k}-u_{0}\right\| \\
& +\left|\left(P\left(z_{k}+h^{\infty}\left(z_{k}\right)+s t u_{k}\right) u_{0}, u_{0}\right)_{H}-\left(P(\infty) u_{0}, u_{0}\right)_{H}\right| \rightarrow 0
\end{aligned}
$$

uniformly in $(s, t) \in[0,1] \times[0,1]$ as $k \rightarrow \infty$. Similarly, from $(2.15)$ we derive that

$$
\left|\left(Q\left(z_{k}+h^{\infty}\left(z_{k}\right)+s t u_{k}\right) u_{k}, u_{k}\right)_{H}-\left(Q(\infty) u_{0}, u_{0}\right)_{H}\right| \rightarrow 0
$$

uniformly in $(s, t) \in[0,1] \times[0,1]$ as $k \rightarrow \infty$. Since $\left(I-P_{\infty}^{0}\right) A\left(z_{k}+h^{\infty}\left(z_{k}\right)\right)=0$ for all $k$, by the mean value theorem we obtain

$$
\begin{aligned}
F^{\infty}\left(z_{k}, u_{k}\right)= & \int_{0}^{1} D \mathcal{L}\left(z_{k}+h^{\infty}\left(z_{k}\right)+t u_{k}\right)\left(u_{k}\right) d t \\
= & \int_{0}^{1}\left(A\left(z_{k}+h^{\infty}\left(z_{k}\right)+t u_{k}\right), u_{k}\right)_{H} d t \\
= & \int_{0}^{1}\left(A\left(z_{k}+h^{\infty}\left(z_{k}\right)+t u_{k}\right)-A\left(z_{k}+h^{\infty}\left(z_{k}\right)\right), u_{k}\right)_{H} d t \\
= & \int_{0}^{1} \int_{0}^{1}\left(B\left(z_{k}+h^{\infty}\left(z_{k}\right)+s t u_{k}\right)\left(t u_{k}\right), u_{k}\right)_{H} d s d t \\
= & \int_{0}^{1} \int_{0}^{1} t\left(P\left(z_{k}+h^{\infty}\left(z_{k}\right)+s t u_{k}\right) u_{k}, u_{k}\right)_{H} d s d t \\
& +\int_{0}^{1} \int_{0}^{1} t\left(Q\left(z_{k}+h^{\infty}\left(z_{k}\right)+s t u_{k}\right) u_{k}, u_{k}\right)_{H} d s d t \\
\rightarrow & \int_{0}^{1} \int_{0}^{1} t\left(P(\infty) u_{0}, u_{0}\right)_{H} d s d t+\int_{0}^{1} \int_{0}^{1} t\left(Q(\infty) u_{0}, u_{0}\right)_{H} d s d t \\
= & \int_{0}^{1} \int_{0}^{1} t\left(B(\infty) u_{0}, u_{0}\right)_{H} d s d t=\frac{1}{2}\left(B(\infty) u_{0}, u_{0}\right)_{H} \quad \text { as } k \rightarrow \infty
\end{aligned}
$$


(b) Since $a_{\infty} \leq\|B(\infty)\|$ and

$\|B(\infty)\| \cdot\left\|h^{\infty}(z)\right\| \cdot\left\|u^{+}+u^{-}\right\| \leq \frac{\|B(\infty)\|^{2}}{2 a_{\infty}}\left\|h^{\infty}(z)\right\|^{2}+\frac{a_{\infty}}{2}\left\|u^{+}\right\|^{2}+\frac{a_{\infty}}{2}\left\|u^{-}\right\|^{2}$,

from (2.12) and (1.1) we derive

$$
\begin{aligned}
\mathcal{L}\left(z+h^{\infty}(z)+\right. & \left.u^{+}+u^{-}\right) \leq \frac{1}{2}\left(B(\infty)\left(h^{\infty}(z)+u^{+}+u^{-}\right), h^{\infty}(z)+u^{+}+u^{-}\right)_{H} \\
& +\frac{a_{\infty}}{8}\left\|z+h^{\infty}(z)+u^{+}+u^{-}\right\|^{2} \\
= & \frac{1}{2}\left(B(\infty) u^{+}, u^{+}\right)_{H}+\frac{1}{2}\left(B(\infty) u^{-}, u^{-}\right)_{H} \\
& +\left(B(\infty) h^{\infty}(z), u^{+}+u^{-}\right)_{H}+\frac{a_{\infty}}{8}\left\|z+h^{\infty}(z)+u^{+}+u^{-}\right\|^{2} \\
\leq & \frac{1}{2}\|B(\infty)\| \cdot\left\|u^{+}\right\|^{2}-a_{\infty}\left\|u^{-}\right\|^{2}+\|B(\infty)\| \cdot\left\|h^{\infty}(z)\right\| \cdot\left\|u^{+}+u^{-}\right\| \\
& +\frac{a_{\infty}}{4}\|z\|^{2}+\frac{a_{\infty}}{4}\left\|h^{\infty}(z)\right\|^{2}+\frac{a_{\infty}}{4}\left\|u^{+}\right\|^{2}+\frac{a_{\infty}}{4}\left\|u^{-}\right\|^{2} \\
\leq & 2\|B(\infty)\| \cdot\left\|u^{+}\right\|^{2}-\frac{a_{\infty}}{4}\left\|u^{-}\right\|^{2}+\frac{a_{\infty}}{4}\|z\|^{2}+\frac{\|B(\infty)\|^{2}}{a_{\infty}}\left\|h^{\infty}(z)\right\|^{2} .
\end{aligned}
$$

Similarly, we have

$$
\begin{aligned}
\mathcal{L}\left(z+h^{\infty}(z)\right. & \left.+u^{+}+u^{-}\right) \geq \frac{1}{2}\left(B(\infty)\left(h^{\infty}(z)+u^{+}+u^{-}\right), h^{\infty}(z)+u^{+}+u^{-}\right)_{H} \\
& -\frac{a_{\infty}}{8}\left\|z+h^{\infty}(z)+u^{+}+u^{-}\right\|^{2} \\
= & \frac{1}{2}\left(B(\infty) u^{+}, u^{+}\right)_{H}+\frac{1}{2}\left(B(\infty) u^{-}, u^{-}\right)_{H} \\
& +\left(B(\infty) h^{\infty}(z), u^{+}+u^{-}\right)_{H}-\frac{a_{\infty}}{8}\left\|z+h^{\infty}(z)+u^{+}+u^{-}\right\|^{2} \\
\geq & a_{\infty}\left\|u^{+}\right\|^{2}-\frac{1}{2}\|B(\infty)\| \cdot\left\|u^{-}\right\|^{2}-\frac{\|B(\infty)\|^{2}}{2 a_{\infty}}\left\|h^{\infty}(z)\right\|^{2}-\frac{a_{\infty}}{2}\left\|u^{+}\right\|^{2} \\
& -\frac{a_{\infty}}{2}\left\|u^{-}\right\|^{2}-\frac{a_{\infty}}{4}\|z\|^{2}-\frac{a_{\infty}}{4}\left\|h^{\infty}(z)\right\|^{2}-\frac{a_{\infty}}{4}\left\|u^{+}\right\|^{2}-\frac{a_{\infty}}{4}\left\|u^{-}\right\|^{2} \\
\geq & \frac{a_{\infty}}{4}\left\|u^{+}\right\|^{2}-2\|B(\infty)\| \cdot\left\|u^{-}\right\|^{2}-\frac{\|B(\infty)\|^{2}}{a_{\infty}} \cdot\left\|h^{\infty}(z)\right\|^{2}-\frac{a_{\infty}}{4}\|z\|^{2} .
\end{aligned}
$$

Hence

$$
\begin{aligned}
\frac{a_{\infty}}{4}\left\|u^{+}\right\|^{2}-2\|B(\infty)\| \cdot\left\|u^{-}\right\|^{2}-\frac{\|B(\infty)\|^{2}}{a_{\infty}} \cdot\left\|h^{\infty}(z)\right\|^{2}-\frac{a_{\infty}}{4}\|z\|^{2} \\
\leq \mathcal{L}\left(z+h^{\infty}(z)+u^{+}+u^{-}\right) \\
\leq 2\|B(\infty)\| \cdot\left\|u^{+}\right\|^{2}-\frac{a_{\infty}}{4}\left\|u^{-}\right\|^{2}+\frac{a_{\infty}}{4}\|z\|^{2}+\frac{\|B(\infty)\|^{2}}{a_{\infty}}\left\|h^{\infty}(z)\right\|^{2} .
\end{aligned}
$$


In particular, we have

$$
\begin{aligned}
-\frac{\|B(\infty)\|^{2}}{a_{\infty}} \cdot\left\|h^{\infty}(z)\right\|^{2}-\frac{a_{\infty}}{4}\|z\|^{2} & \leq \mathcal{L}\left(z+h^{\infty}(z)\right) \\
& \leq \frac{a_{\infty}}{4}\|z\|^{2}+\frac{\|B(\infty)\|^{2}}{a_{\infty}}\left\|h^{\infty}(z)\right\|^{2} .
\end{aligned}
$$

Since $F^{\infty}\left(z, u^{+}+u^{-}\right)=\mathcal{L}\left(z+h^{\infty}(z)+u^{+}+u^{-}\right)-\mathcal{L}\left(z+h^{\infty}(z)\right)$ by $(2.10)$, the desired inequalities easily follow.

For $F^{\infty}$ we can directly prove the corresponding conclusions with Step 1 in the proof of Theorem 2.1 (given in Appendix A of [13], [14]) as follows.

LEMmA 2.8. (a) For any $r \in(0, \infty)$ there exists a number $\varepsilon_{r} \in(0, r)$ such that for each $(z, u) \in \bar{B}_{H_{\infty}^{0}}\left(\infty, R_{1}\right) \times \bar{B}_{H_{\infty}^{+}}\left(\theta, \varepsilon_{r}\right)$ there exists a unique point $\varphi_{z}(u) \in B_{H_{\infty}^{-}}(\theta, r)$ satisfying

$$
F^{\infty}\left(z, u+\varphi_{z}(u)\right)=\max \left\{F^{\infty}(z, u+v) \mid v \in B_{H_{\infty}^{-}}(\theta, r)\right\} .
$$

One has also $\varphi_{z}(\theta)=\theta$.

(b) If $\mathcal{L}(u)=(B(\infty) u, u)_{H} / 2+o\left(\|u\|^{2}\right)$ as $\|u\| \rightarrow \infty$, for each $(z, u)$ in $\bar{B}_{H_{\infty}^{0}}\left(\infty, R_{1}\right) \times H_{\infty}^{+}$there exists a unique point $\varphi_{z}(u) \in H_{\infty}^{-}$such that

$$
F^{\infty}\left(z, u+\varphi_{z}(u)\right)=\max \left\{F^{\infty}(z, u+v) \mid v \in H_{\infty}^{-}\right\} .
$$

Moreover, $\varphi_{z}(\theta)=\theta$, and

$$
\left\|\varphi_{z}\left(u^{+}\right)\right\|^{2} \leq \frac{8}{a_{\infty}}\|B(\infty)\| \cdot\left\|u^{+}\right\|^{2}+4\|z\|^{2}+\frac{16\|B(\infty)\|^{2}}{a_{\infty}^{2}}\left\|h^{\infty}(z)\right\|^{2} .
$$

Clearly, Lemma 2.7(b) implies that for any bounded subset $K \subset \bar{B}_{H_{\infty}^{0}}\left(R_{1}, \infty\right)$, $F^{\infty}\left(z, u+\varphi_{z}(u)\right) \geq F^{\infty}(z, u) \rightarrow \infty$ uniformly in $z \in K$ as $u \in H_{\infty}^{+}$and $\|u\| \rightarrow \infty$.

Later on we shall understand $r=\infty$ and $\varepsilon_{\infty}=\infty$ for conveniences in case (b). Note that the cases (a) and (b) of Lemma 2.8 correspond to Theorems 1.3 and 1.1, respectively. Moreover, if Lemmas 2.4-2.6 only hold in a set $\bar{B}_{H_{\infty}^{0}}\left(\infty, R^{\prime}\right) \oplus$ $\left(\bar{B}_{H}\left(\theta, r^{\prime}\right) \cap X_{\infty}^{ \pm}\right)$, then $z$ and $r$ in (a) are restricted in $\bar{B}_{H_{\infty}^{0}}\left(\infty, R^{\prime}\right)$ and $\left(0, r^{\prime}\right)$, respectively.

Proof of Lemma 2.8. As at the beginning of proof of Theorem 2.1 (given in Appendix A of [13], [14]) we only need to consider the case $\operatorname{dim} H_{\infty}^{-}>0$.

(a) Since the function $H_{\infty}^{-} \rightarrow \mathbb{R}, u^{-} \mapsto F^{\infty}\left(z, u^{+}+u^{-}\right)$is strictly concave by Lemma 2.6(b), it has a unique maximum point on a convex set if existing. Clearly, it attains the maximum on the compact subset $\bar{B}_{H_{\infty}^{-}}(\theta, r)$. Suppose by contradiction that there exist sequences $\left\{\left(z_{n}, x_{n}\right)\right\} \in \bar{B}_{H_{\infty}^{0}}\left(\infty, R_{1}\right) \times \bar{B}_{H_{\infty}^{+}}(\theta, r)$ with $x_{n} \rightarrow 0$, and $\left\{v_{n}\right\} \subset \partial \bar{B}_{H_{\infty}^{-}}(\theta, r)$ such that

(2.16) $F^{\infty}\left(z_{n}, x_{n}+v_{n}\right)>F^{\infty}\left(z_{n}, x_{n}+u\right)$ for all $u \in B_{H_{\infty}^{-}}(\theta, r)$, for all $n \in \mathbb{N}$. 
If $\left\{z_{n}\right\}$ is bounded we may assume up to subsequences that $z_{n} \rightarrow z_{0} \in$ $\bar{B}_{H_{\infty}^{0}}\left(\infty, R_{1}\right)$ and $v_{n} \rightarrow v_{0} \in \partial \bar{B}_{H_{\infty}^{-}}(\theta, r)$ since both $\bar{B}_{H_{\infty}^{0}}\left(\infty, R_{1}\right)$ and $\partial \bar{B}_{H_{\infty}^{-}}(\theta, r)$ are compact. It follows from these and (2.16) that

$$
F^{\infty}\left(z_{0}, v_{0}\right) \geq F^{\infty}\left(z_{0}, u\right) \text { for all } u \in B_{H_{\infty}^{-}}(\theta, r) .
$$

On the other hand, the mean value theorem yields a number $s \in(0,1)$ such that

$$
\begin{aligned}
F^{\infty}\left(z_{0}, v_{0}\right) & =F^{\infty}\left(z_{0}, v_{0}\right)-F^{\infty}\left(z_{0}, \theta\right)=D_{2} F\left(z_{0}, s v_{0}\right) v_{0} \\
& =\frac{1}{s}\left[D_{2} F\left(z_{0}, s v_{0}\right)\left(s v_{0}\right)-D_{2} F\left(z_{0}, \theta\right)\left(s v_{0}\right)\right] \\
& \leq-\frac{a_{\infty}}{s}\left\|s v_{0}\right\|^{2}=-s a_{\infty}\left\|v_{0}\right\|^{2}<0=F^{\infty}\left(z_{0}, \theta\right)
\end{aligned}
$$

by Lemma 2.6(a)-(b). A contradiction is obtained in this case.

Up to subsequences we assume that $\left\|z_{n}\right\| \rightarrow \infty$ and $v_{n} \rightarrow v_{0} \in \partial \bar{B}_{H_{\infty}^{-}}(\theta, r)$ in $H$. Then $H_{\infty}^{ \pm} \ni u_{n}:=x_{n}+v_{n} \rightarrow v_{0}$. By Lemma 2.7 we get

$$
F^{\infty}\left(z_{n}, x_{n}+v_{n}\right) \rightarrow \frac{1}{2}\left(B(\infty) v_{0}, v_{0}\right)_{H}<0, \quad F^{\infty}\left(z_{n}, x_{n}\right) \rightarrow \frac{1}{2}(B(\infty) \theta, \theta)_{H}=0 .
$$

Hence (2.16) leads to $\left(B(\infty) v_{0}, v_{0}\right)_{H} \geq 0$, and therefore a contradiction is obtained again.

To see $\varphi_{z}(\theta)=\theta$, note that $D_{2} F^{\infty}\left(z, \varphi_{z}(\theta)\right)=0$. If $\varphi_{z}(\theta) \neq \theta$ then

$$
0=\left[D_{2} F^{\infty}\left(z, \varphi_{z}(\theta)\right)-D_{2} F^{\infty}(z, \theta)\right]\left(\varphi_{z}(\theta)-\theta\right) \leq-a_{\infty}\left\|\varphi_{z}(\theta)\right\|^{2}<0
$$

by Lemma 2.6(b), which is a contradiction.

(b) By Lemma 2.6(b) the function $H_{\infty}^{-} \rightarrow \mathbb{R}, u^{-} \mapsto-F^{\infty}\left(z, u^{+}+u^{-}\right)$is strictly convex. The second claim of Lemma 2.7 also shows that this function is coercive. Hence it attains the minimum at some point $\varphi_{z}\left(u^{+}\right) \in H_{\infty}^{-}$. That is, the function $H_{\infty}^{-} \rightarrow \mathbb{R}, u^{-} \mapsto F^{\infty}\left(z, u^{+}+u^{-}\right)$takes the maximum at $\varphi_{z}\left(u^{+}\right)$. As in the proof of Lemma 2.1 of [9] the uniqueness of $\varphi_{z}\left(u^{+}\right)$follows from Lemma 2.6(b) as well.

The proof that $\varphi_{z}(\theta)=\theta$ may be obtained as above. To see the another claim, by Lemma $2.7(\mathrm{~b})$,

$$
\begin{aligned}
2\|B(\infty)\| \cdot \| u^{+} & \left\|^{2}-\frac{a_{\infty}}{4}\right\| \varphi_{z}\left(u^{+}\right)\left\|^{2}+\frac{a_{\infty}}{2}\right\| z\left\|^{2}+\frac{2\|B(\infty)\|^{2}}{a_{\infty}}\right\| h^{\infty}(z) \|^{2} \\
& \geq F^{\infty}\left(z, u^{+}+\varphi_{z}\left(u^{+}\right)\right) \geq F^{\infty}\left(z, u^{+}\right) \\
& \geq \frac{a_{\infty}}{4}\left\|u^{+}\right\|^{2}-\frac{2\|B(\infty)\|^{2}}{a_{\infty}} \cdot\left\|h^{\infty}(z)\right\|^{2}-\frac{a_{\infty}}{2}\|z\|^{2} .
\end{aligned}
$$

The conclusion follows immediately.

REMARK 2.9. Note that a local maximum of a concave function (with finite values) on a normed linear space is also a global maximum. From Lemma 2.8(a) it follows that for any $r>0$ there exists a number $\varepsilon_{r} \in(0, r)$ such that for each 
$(z, u) \in \bar{B}_{H_{\infty}^{0}}\left(\infty, R_{1}\right) \times \bar{B}_{H_{\infty}^{+}}\left(\theta, \varepsilon_{r}\right)$ there exists a unique point $\varphi_{z}(u) \in B_{H_{\infty}^{-}}(\theta, r)$ satisfying

$$
\begin{aligned}
F^{\infty}\left(z, u+\varphi_{z}(u)\right) & =\max \left\{F^{\infty}(z, u+v) \mid v \in B_{H_{\infty}^{-}}(\theta, r)\right\} \\
& =\max \left\{F^{\infty}(z, u+v) \mid v \in H_{\infty}^{-}\right\} .
\end{aligned}
$$

Define

$$
r_{\mathcal{L}}:=\sup \left\{\varepsilon_{r} \mid r>0\right\} .
$$

Then for each $(z, u) \in \bar{B}_{H_{\infty}^{0}}\left(\infty, R_{1}\right) \times B_{H_{\infty}^{+}}\left(\theta, r_{\mathcal{L}}\right)$ there exists a unique point $\varphi_{z}(u) \in H_{\infty}^{-}$with $\varphi_{z}\left(\theta^{+}\right)=\theta^{-}$, such that

$$
F^{\infty}\left(z, u+\varphi_{z}(u)\right)=\max \left\{F^{\infty}(z, u+v) \mid v \in H_{\infty}^{-}\right\} .
$$

Clearly, under the assumption (1.5), i.e. $\mathcal{L}(u)=(B(\infty) u, u)_{H} / 2+o\left(\|u\|^{2}\right)$ as $\|u\| \rightarrow \infty$, we have $r_{\mathcal{L}}=\infty$ by Lemma 2.8(b) (because $\varepsilon_{\infty}=\infty$ ). [Note: if Lemmas 2.4-2.6 only hold in a set $\bar{B}_{H_{\infty}^{0}}\left(\infty, R^{\prime}\right) \oplus\left(\bar{B}_{H}\left(\theta, r^{\prime}\right) \cap X_{\infty}^{ \pm}\right)$, we define $r_{\mathcal{L}}:=\sup \left\{\varepsilon_{r} \mid 0<r<r^{\prime}\right\}$. Then for each $(z, u) \in \bar{B}_{H_{\infty}^{0}}\left(\infty, R^{\prime}\right) \times B_{H_{\infty}^{+}}\left(\theta, r_{\mathcal{L}}\right)$ there exists a unique $\varphi_{z}(u) \in B_{H_{\infty}^{-}}\left(\theta, r^{\prime}\right)$ with $\varphi_{z}\left(\theta^{+}\right)=\theta^{-}$, such that $F^{\infty}(z, u+$ $\left.\varphi_{z}(u)\right)=\max \left\{F^{\infty}(z, u+v) \mid v \in B_{H_{\infty}^{-}}\left(\theta, r^{\prime}\right)\right\}$. In this case the following map $j$ is only defined on $\bar{B}_{H_{\infty}^{0}}\left(\infty, R^{\prime}\right) \times B_{H_{\infty}^{+}}\left(\theta, r_{\mathcal{L}}\right)$.]

It is easily seen that the following map

$$
j: \bar{B}_{H_{\infty}^{0}}\left(\infty, R_{1}\right) \times B_{H_{\infty}^{+}}\left(\theta, r_{\mathcal{L}}\right) \rightarrow \mathbb{R}, \quad(z, u) \mapsto F^{\infty}\left(z, u+\varphi_{z}(u)\right),
$$

is well-defined.

Lemma 2.10. The map $j$ is continuous, and for every $z \in \bar{B}_{H_{\infty}^{0}}\left(\infty, R_{1}\right)$ the map $B_{H_{\infty}^{+}}\left(\theta, r_{\mathcal{L}}\right) \rightarrow \mathbb{R}, u \mapsto j(z, u)$ is continuously directional differentiable.

Proof. Clearly, it suffices to prove that the restriction of $j$ to $\bar{B}_{H_{\infty}^{0}}\left(\infty, R_{1}\right) \times$ $B_{H_{\infty}^{+}}\left(\theta, \varepsilon_{r}\right)$ is continuously directional differentiable.

If $r<\infty$, since $\bar{B}_{H}\left(\infty, R_{1}\right) \cap \bar{B}_{H}(\theta, R) \cap H_{\infty}^{0}$ is compact for any $R>R_{1}$, as in Step 3 of the proof of Theorem 2.1 (given in Appendix A of [13, 14]) we can get the desired conclusion from Lemma 2.3 of [9].

If $r=\infty$, i.e. (1.5) holds, for any $\left(z_{0}, u_{0}^{+}\right) \in \bar{B}_{H_{\infty}^{0}}\left(\infty, R_{1}\right) \times H_{\infty}^{+}$, by Lemma 2.8(b) there exists a bounded neighbourhood $\mathcal{U}$ of it in $\bar{B}_{H_{\infty}^{0}}\left(\infty, R_{1}\right) \times$ $H_{\infty}^{+}$and a positive number $R$ such that $\varphi_{z}(u) \in B_{H_{\infty}^{0}}(\theta, R)$ for all $(z, u) \in \mathcal{U}$. Suppose that $\left\{\left(z_{n}, u_{n}^{+}\right)\right\}$converges to $\left(z_{0}, u_{0}^{+}\right)$. As in Step 2 of the proof of Theorem 2.1 (given in Appendix A of [13], [14]) it is easily proved that $\varphi_{z_{n}}\left(u_{n}^{+}\right) \rightarrow$ $\varphi_{z_{0}}\left(u_{0}^{+}\right)$as $n \rightarrow \infty$. Hence $j$ is continuous in this case. The second claim follows from Lemma 2.3 of [9].

By $(2.17)$, for $(z, u) \in \bar{B}_{H_{\infty}^{0}}\left(\infty, R_{1}\right) \times B_{H_{\infty}^{+}}\left(\theta, r_{\mathcal{L}}\right)$ we have

$$
F^{\infty}\left(z, u+\varphi_{z}(u)\right) \geq F^{\infty}(z, u+v) \text { for all } v \in H_{\infty}^{-} .
$$


Moreover, for any $z \in \bar{B}_{H_{\infty}^{0}}\left(\infty, R_{1}\right)$ we have also

$$
\begin{array}{ll}
F^{\infty}(z, u) \geq \frac{a_{1}}{4}\|u\|^{2} & \text { for all } u \in H_{\infty}^{+}, \\
F^{\infty}(z, v) \leq-\frac{a_{\infty}}{4}\|v\|^{2} & \text { for all } v \in H_{\infty}^{-} .
\end{array}
$$

In fact, using the mean value theorem and Lemma 2.6(iv) we get

$$
\begin{aligned}
F^{\infty}(z, u) & =F^{\infty}(z, u)-F^{\infty}(z, \theta)=D_{2} F^{\infty}(z, s u)(u) \\
& =\frac{1}{s} D_{2} F^{\infty}(z, s u)(s u) \geq a_{1} s\|u\|^{2} \geq 0
\end{aligned}
$$

for some $s \in(0,1)$. If $u \neq \theta$, the same reason yields a number $s_{u} \in(1 / 2,1)$ such that

$$
F^{\infty}(z, u)>F^{\infty}(z, u)-F^{\infty}(z, u / 2)=D_{2} F^{\infty}\left(z, s_{u} u\right)(u / 2) \geq \frac{a_{1}}{4}\|u\|^{2} .
$$

Similarly, we get a number $s \in(0,1)$ such that

$$
\begin{aligned}
F^{\infty}(z, v) & =F^{\infty}(z, v)-F^{\infty}(z, \theta)=D_{2} F^{\infty}(z, s v)(v) \\
& =\frac{1}{s} D_{2} F^{\infty}(z, s v)(s v) \leq-a_{\infty} s\|v\|^{2} \leq 0
\end{aligned}
$$

by Lemma 2.6(c). Moreover, if $v \neq \theta$ we have also a number $s_{v} \in(1 / 2,1)$ such that

$$
F^{\infty}(z, v)<F^{\infty}(z, v)-F^{\infty}(z, v / 2)=D_{2} F^{\infty}\left(z, s_{v} v\right)(v / 2) \leq-\frac{a_{\infty}}{4}\|v\|^{2} .
$$

For $r \in(0, \infty], z \in \bar{B}_{H_{\infty}^{0}}\left(\infty, R_{1}\right)$ and $(u, v) \in \bar{B}_{H_{\infty}^{+}}\left(\theta, \varepsilon_{r}\right) \times B_{H_{\infty}^{-}}(\theta, r)$, define

$$
\begin{aligned}
& \psi_{1}(z, u+v)= \begin{cases}\frac{\sqrt{F^{\infty}\left(z, u+\varphi_{z}(u)\right)}}{\|u\|} & \text { if } u \neq \theta, \\
\theta & \text { if } u=\theta,\end{cases} \\
& \psi_{2}(z, u+v)= \begin{cases}\frac{\sqrt{F^{\infty}\left(z, u+\varphi_{z}(u)\right)-F^{\infty}(z, u+v)}}{\left\|v-\varphi_{z}(u)\right\|}\left(v-\varphi_{z}(u)\right) & \text { if } v \neq \varphi_{z}(u), \\
\theta & \text { if } v=\varphi_{z}(u) .\end{cases}
\end{aligned}
$$

By Lemma 2.10, the map

$$
\psi: \bar{B}_{H_{\infty}^{0}}\left(\infty, R_{1}\right) \times\left(\bar{B}_{H_{\infty}^{+}}\left(\theta, \varepsilon_{r}\right) \oplus B_{H_{\infty}^{-}}(\theta, r)\right) \rightarrow H_{\infty}^{ \pm}
$$

given by $\psi(z, u+v)=\psi_{1}(z, u+v)+\psi_{2}(z, u+v)$, is continuous. Clearly,

$$
\psi(z, u+v) \in \operatorname{Im}(\psi) \cap H_{\infty}^{-} \text {if and only if } u=\theta,
$$

$$
\text { and } F^{\infty}(z, u+v)=\left\|\psi_{1}(z, u+v)\right\|^{2}-\left\|\psi_{2}(z, u+v)\right\|^{2} .
$$

As in Step 5 in the proof of Theorem 2.1 (given in Appendix A of [13], [14]) we can prove 
LEMma 2.11. For each $z \in \bar{B}_{H_{\infty}^{0}}\left(\infty, R_{1}\right)$ the map

$$
\psi(z, \cdot): \bar{B}_{H_{\infty}^{+}}\left(\theta, \varepsilon_{r}\right) \oplus B_{H_{\infty}^{-}}(\theta, r) \rightarrow H_{\infty}^{ \pm}
$$

is injective whether $r$ is finite or infinite.

[Note: If Lemmas $2.4-2.6$ only hold in a set $\bar{B}_{H_{\infty}^{0}}\left(\infty, R^{\prime}\right) \oplus\left(\bar{B}_{H}\left(\theta, r^{\prime}\right) \cap X_{\infty}^{ \pm}\right)$, we require $z$ and $r$ in this lemma and the following Lemma 2.12(a) to sit in $\bar{B}_{H_{\infty}^{0}}\left(\infty, R^{\prime}\right)$ and $\left(0, r^{\prime}\right)$, respectively.]

Now we are a position to prove the corresponding conclusions with Step 6 in the proof of Theorem 2.1 (given in Appendix A of [13], [14]).

LEMma 2.12. (a) For any $r \in(0, \infty)$ there is a number $\epsilon_{r} \in\left(0, \varepsilon_{r} / 4\right)$ such that

$$
B_{H_{\infty}^{+}}\left(\theta, \sqrt{a_{1}} \epsilon_{r}\right) \oplus B_{H_{\infty}^{-}}\left(\theta, \sqrt{a_{1}} \epsilon_{r}\right) \subset \psi\left(z, B_{H_{\infty}^{+}}\left(\theta, 2 \epsilon_{r}\right) \oplus B_{H_{\infty}^{-}}(\theta, r)\right)
$$

for any $z \in \bar{B}_{H_{\infty}^{0}}\left(\infty, R_{1}\right)$.

(b) If $\mathcal{L}(u)=(B(\infty) u, u)_{H} / 2+o\left(\|u\|^{2}\right)$ as $\|u\| \rightarrow \infty$, that is, $r=\infty$, then for each $z \in \bar{B}_{H_{\infty}^{0}}\left(\infty, R_{1}\right)$ the map

$$
\psi(z, \cdot): H_{\infty}^{+} \oplus H_{\infty}^{-} \rightarrow H_{\infty}^{+} \oplus H_{\infty}^{-}
$$

is surjective, and hence bijective due to Lemma 2.11. As a consequence we get

$$
\psi^{-1}\left(H_{\infty}^{+} \oplus H_{\infty}^{-}\right)=\bar{B}_{H_{\infty}^{0}}\left(\infty, R_{1}\right) \times\left(H_{\infty}^{+} \oplus H_{\infty}^{-}\right) .
$$

Proof. (a) By (2.22) there exists a number $C>0$ such that

$$
F^{\infty}(z, v)<-C \text { for all }(z, v) \in \bar{B}_{H_{\infty}^{0}}\left(\infty, R_{1}\right) \times \partial B_{H_{\infty}^{-}}(\theta, r) .
$$

Claim 2.12.1. There exists a number $\epsilon_{r} \in\left(0, \varepsilon_{r} / 4\right)$ such that

$$
F^{\infty}(z, u+v) \leq 0
$$

for any $(z, u, v) \in \bar{B}_{H_{\infty}^{0}}\left(\infty, R_{1}\right) \times \bar{B}_{H_{\infty}^{+}}\left(\theta, 2 \epsilon_{r}\right) \times \partial B_{H_{\infty}^{-}}(\theta, r)$.

Suppose by contradiction that there exists a sequence

$$
\left\{\left(z_{n}, u_{n}, v_{n}\right)\right\} \subset \bar{B}_{H_{\infty}^{0}}\left(\infty, R_{1}\right) \times \bar{B}_{H_{\infty}^{+}}\left(\theta, \varepsilon_{r}\right) \times \partial B_{H_{\infty}^{-}}(\theta, r)
$$

such that $u_{n} \rightarrow \theta$ and $F^{\infty}\left(z_{n}, u_{n}+v_{n}\right) \geq 0$ for all $n$. If $\left\{z_{n}\right\}$ has a bounded subsequence we can get a contradiction as in Step 6 of proof of Theorem 2.1 (given in Appendix A of [13], [14]). Otherwise, after passing to a subsequence we may assume that $\left\|z_{n}\right\| \rightarrow \infty$ and $v_{n} \rightarrow v_{0}$. Then using Lemma 2.7(a) we derive

$$
F\left(z_{k}, u_{k}+v_{k}\right) \rightarrow \frac{1}{2}\left(B(\infty) v_{0}, v_{0}\right)_{H}<0 \quad \text { as } k \rightarrow \infty .
$$

This leads to a contradiction again. (2.25) is proved.

Claim 2.12.2. One can shrink the positive number $\epsilon_{r}$ in $(2.25)$ such that

$$
\varphi_{z}\left(\bar{B}_{H_{\infty}^{+}}\left(\theta, 2 \epsilon_{r}\right)\right) \subset B_{H_{\infty}^{-}}(\theta, r / 2) \quad \text { for all } z \in \bar{B}_{H_{\infty}^{0}}\left(\infty, R_{1}\right)
$$


By a contradiction suppose that there exist sequences $\left\{z_{n}\right\} \subset \bar{B}_{H_{\infty}^{0}}\left(\infty, R_{1}\right)$ and $\left\{u_{n}\right\} \subset \bar{B}_{H_{\infty}^{+}}\left(\theta, \varepsilon_{r}\right)$ such that

$$
\left\|u_{n}\right\| \rightarrow 0 \quad \text { and } \quad \varphi_{z_{n}}\left(u_{n}\right) \notin B_{H_{\infty}^{-}}(\theta, r / 2) \quad \text { for all } n=1,2, \ldots
$$

By Lemma 2.8 each $\varphi_{z_{n}}\left(u_{n}\right)$ is a unique point in $B_{H_{\infty}^{-}}(\theta, r)$ such that

$$
F^{\infty}\left(z_{n}, u_{n}+\varphi_{z_{n}}\left(u_{n}\right)\right)=\max \left\{F^{\infty}\left(z_{n}, u_{n}+v\right) \mid v \in B_{H_{\infty}^{-}}(\theta, r)\right\} .
$$

Since $\bar{B}_{H_{\infty}^{-}}(\theta, r)$ is compact, after passing a subsequence (if necessary) we may assume $\varphi_{z_{n}}\left(u_{n}\right) \rightarrow v_{0} \in \bar{B}_{H_{\infty}^{-}}(\theta, r) \backslash B_{H_{\infty}^{-}}(\theta, r / 2)$.

- If $\left\{z_{n}\right\}$ has a bounded subsequence, passing to a subsequence we may assume $z_{n} \rightarrow z_{0} \in \bar{B}_{H_{\infty}^{0}}\left(\infty, R_{1}\right)$. Then by $(2.22)$ we get

$$
F^{\infty}\left(z_{n}, u_{n}+\varphi_{z_{n}}\left(u_{n}\right)\right) \rightarrow F^{\infty}\left(z_{0}, v_{0}\right) \leq-\frac{a_{\infty}}{4}\left\|v_{0}\right\|^{2} \leq-\frac{r^{2} a_{\infty}}{16}<0
$$

as $n \rightarrow \infty$, and $F^{\infty}\left(z_{n}, u_{n}\right) \rightarrow F^{\infty}\left(z_{0}, \theta\right)=0$ as $n \rightarrow \infty$. This contradicts to the fact that $F^{\infty}\left(z_{n}, u_{n}\right) \leq F^{\infty}\left(z_{n}, u_{n}+\varphi_{z_{n}}\left(u_{n}\right)\right)$ for all $n$.

- If $\left\{z_{n}\right\}$ has no bounded subsequences, passing to a subsequence we may assume $\left\|z_{n}\right\| \rightarrow \infty$. In this case Lemma 2.7(a) leads to

$$
F^{\infty}\left(z_{n}, u_{n}+\varphi_{z_{n}}\left(u_{n}\right)\right) \rightarrow \frac{1}{2}\left(B(\infty) v_{0}, v_{0}\right)_{H} \leq-a_{\infty}\left\|v_{0}\right\|^{2} \leq-\frac{a_{\infty} r^{2}}{4}
$$

as $n \rightarrow \infty$, and $F^{\infty}\left(z_{n}, u_{n}\right) \rightarrow(B(\infty) \theta, \theta)_{H} / 2=0$ as $n \rightarrow \infty$. This also yields a contradiction to the fact that $F^{\infty}\left(z_{n}, u_{n}+\varphi_{z_{n}}\left(u_{n}\right)\right) \geq F^{\infty}\left(z_{n}, u_{n}\right)$ for all $n$.

Claim 2.12.2 is proved.

For $(z, u) \in \bar{B}_{H_{\infty}^{0}}\left(\infty, R_{1}\right) \times B_{H_{\infty}^{+}}\left(\theta, 2 \epsilon_{r}\right)$, by (2.20) and (2.21) we get

$$
F^{\infty}\left(z, u+\varphi_{z}(u)\right) \geq F^{\infty}(z, u) \geq \frac{a_{1}}{4}\|u\|^{2} .
$$

This and (2.25) imply that

$$
F^{\infty}\left(z, u+\varphi_{z}(u)\right)-F^{\infty}(z, u+v)>a_{1} \epsilon_{r}^{2}
$$

for any $(z, u, v) \in \bar{B}_{H_{\infty}^{0}}\left(\infty, R_{1}\right) \times \partial B_{H_{\infty}^{+}}\left(\theta, 2 \epsilon_{r}\right) \times \partial B_{H_{\infty}^{-}}(\theta, r)$.

Note that (2.24)-(2.28) correspond to (A.2)-(A.6) in Step 6 in the proof of Theorem 2.1 (given in Appendix A of [13], [14]), respectively. Using these and repeating the remained arguments therein (i.e. Step 6 in the proof of Theorem 2.1 given in Appendix A of [13], [14]) we may get

$$
\bar{B}_{H_{\infty}^{+}}\left(\theta, \sqrt{a_{1}} \epsilon_{r}\right) \subset \psi_{1}\left(z, B_{H_{\infty}^{+}}\left(\theta, 2 \epsilon_{r}\right)\right)
$$

and the desired conclusion (a).

(b) For any given $\left(\bar{u}^{+}, \bar{u}^{-}\right) \in H_{\infty}^{+} \times H_{\infty}^{-}$, without loss of generality, we assume $\left(\bar{u}^{+}, \bar{u}^{-}\right) \neq(\theta, \theta)$ because $\psi(z, \theta)=\theta$.

- If $\bar{u}^{+}=\theta$ then $\bar{u}^{-} \neq \theta$. Since (2.17) and Lemma 2.7(b) imply

$$
0=F^{\infty}\left(z, \varphi_{z}(\theta) \geq F^{\infty}(z, u) \rightarrow-\infty \quad \text { as } u \in H_{\infty}^{-} \text {and }\|u\| \rightarrow \infty,\right.
$$


the intermediate value theorem gives a number $t>0$ such that $-F^{\infty}\left(z, t \bar{u}^{-}\right)=$ $\left\|\bar{u}^{-}\right\|^{2}$. Set $u^{-}:=t \bar{u}^{-}$. Then $\psi_{1}\left(z, \theta+u^{-}\right)=\psi_{1}(z, \theta)=\theta$ and

$$
\psi_{2}\left(z, \theta+u^{-}\right)=\frac{\sqrt{F^{\infty}\left(z, \varphi_{z}(\theta)\right)-F^{\infty}\left(z, u^{-}\right)}}{\left\|u^{-}-\varphi_{z}(\theta)\right\|}\left(u^{-}-\varphi_{z}(\theta)\right)=\bar{u}^{-} .
$$

Namely, $\psi\left(z, \theta+u^{-}\right)=\left(\theta, \bar{u}^{-}\right)$.

- Let $\bar{u}^{+} \neq \theta$. By Lemma 2.8(b), $\varphi_{z}(\theta)=\theta$ and $F^{\infty}\left(z, u+\varphi_{z}(u)\right) \rightarrow \infty$ as $u \in$ $H_{\infty}^{+}$and $\|u\| \rightarrow \infty$. Lemma 2.10 also tells us that $H_{\infty}^{+} \ni u \mapsto F^{\infty}\left(z, u+\varphi_{z}(u)\right)$ is continuous. By the intermediate value theorem we have a number $t>0$ such that

$$
F^{\infty}\left(z, t \bar{u}^{+}+\varphi_{z}\left(t \bar{u}^{+}\right)\right)=\left\|\bar{u}^{+}\right\|^{2} .
$$

Set $u^{+}:=t \bar{u}^{+}$. Then $\psi_{1}\left(z, u^{+}+v\right)=\bar{u}^{+}$for any $v \in H_{\infty}^{-}$. If $\bar{u}^{-}=\theta$, then

$$
\psi_{2}\left(z, u^{+}+u^{-}\right)=\theta=\bar{u}^{-} \quad \text { for } u^{-}=\varphi_{z}\left(u^{+}\right) .
$$

If $\bar{u}^{-} \neq \theta$, we define a function $g:[0, \infty) \rightarrow \mathbb{R}$ by

$$
g(s)=F^{\infty}\left(z, u^{+}+\varphi_{z}\left(u^{+}\right)\right)-F^{\infty}\left(z, u^{+}+\varphi_{z}\left(u^{+}\right)+s \bar{u}^{-}\right) .
$$

Then $g(s) \geq 0, g(0)=0$ and $g(s) \rightarrow \infty$ as $s \rightarrow \infty$ by Lemma 2.7(b). Using the intermediate value theorem may yield a number $s_{0}>0$ such that $g\left(s_{0}\right)=\left\|\bar{u}^{-}\right\|^{2}$. Hence for $u^{-}:=\varphi_{z}\left(u^{+}\right)+s_{0} \bar{u}^{-} \in H_{\infty}^{-}$we get

$$
\frac{\sqrt{F^{\infty}\left(z, u^{+}+\varphi_{z}\left(u^{+}\right)\right)-F^{\infty}\left(z, u^{+}+u^{-}\right)}}{\left\|u^{-}-\varphi_{z}\left(u^{+}\right)\right\|}\left(u^{-}-\varphi_{z}\left(u^{+}\right)\right)=\bar{u}^{-} .
$$

This shows $\psi\left(z, u^{+}+u^{-}\right)=\left(\bar{u}^{+}, \bar{u}^{-}\right)$.

Summarizing the above arguments we have proved that the map $\psi(z, \cdot)$ is surjective. The other conclusions of (b) easily follows.

The cases (a) and (b) of Lemma 2.12 correspond to Theorems 1.3 and 1.1, respectively. If Lemmas 2.4-2.6 only hold in a set $\bar{B}_{H_{\infty}^{0}}\left(\infty, R^{\prime}\right) \oplus\left(\bar{B}_{H}\left(\theta, r^{\prime}\right) \cap\right.$ $\left.X_{\infty}^{ \pm}\right)$, we require $z$ and $r$ in Lemma $2.12\left(\right.$ a) to sit in $\bar{B}_{H_{\infty}^{0}}\left(\infty, R^{\prime}\right)$ and $\left(0, r^{\prime}\right)$, respectively.

The following two lemmas give the corresponding conclusions with Step 7 of the proof of Theorem 2.1 (given in Appendix A of [13], [14]) in the cases of Theorems 1.1 and 1.3, respectively.

Lemma 2.13. Let $\mathcal{L}(u)=(B(\infty) u, u)_{H} / 2+o\left(\|u\|^{2}\right)$ as $\|u\| \rightarrow \infty$. (That is, $r=\infty)$. By Lemmas 2.11 and 2.12(b) we have a bijection

$$
\begin{gathered}
\bar{B}_{H_{\infty}^{0}}\left(\infty, R_{1}\right) \times\left(H_{\infty}^{+} \oplus H_{\infty}^{-}\right) \rightarrow \bar{B}_{H_{\infty}^{0}}\left(\infty, R_{1}\right) \times\left(H_{\infty}^{+} \oplus H_{\infty}^{-}\right), \\
(z, u+v) \mapsto(z, \psi(z, u+v)) .
\end{gathered}
$$

Its inverse, denoted by $\phi$, has a form

$$
\phi(z, u+v)=\left(z, \phi_{z}(u+v)\right):=\left(z, u^{\prime}+v^{\prime}\right),
$$


where $\left(u^{\prime}, v^{\prime}\right) \in H_{\infty}^{+} \times H_{\infty}^{-}$is a unique point satisfying $u+v=\psi\left(z, u^{\prime}+v^{\prime}\right)$. Then $\phi$ is a homeomorphism and

$$
F^{\infty}(\phi(z, u+v))=\|u\|^{2}-\|v\|^{2}
$$

for any $(z, u, v) \in \bar{B}_{H_{\infty}^{0}}\left(\infty, R_{1}\right) \times H_{\infty}^{+} \times H_{\infty}^{-}$. In particular, for each $z \in$ $\bar{B}_{H_{\infty}^{0}}\left(\infty, R_{1}\right), \phi_{z}$ (and so $\left.\psi_{z}=\psi(z, \cdot)\right)$ is a homeomorphism from $H_{\infty}^{+} \oplus H_{\infty}^{-}$ onto $H_{\infty}^{+} \oplus H_{\infty}^{-}$. Moreover, $\phi(z, u+v)$ belongs to $\operatorname{Im}(\psi) \cap\left(\bar{B}_{H_{\infty}^{0}}\left(\infty, R_{1}\right) \times H_{\infty}^{-}\right)$ if and only if $u=\theta$.

Proof. By Lemma 2.12(b) it suffices to prove that $\phi$ is continuous. Suppose that

$$
\begin{aligned}
\left(z_{0}, u_{0}^{\prime}, v_{0}^{\prime}\right) & \in \bar{B}_{H_{\infty}^{0}}\left(\infty, R_{1}\right) \times H_{\infty}^{+} \times H_{\infty}^{-}, \\
\left\{\left(z_{n}, u_{n}^{\prime}, v_{n}^{\prime}\right)\right\} & \subset \bar{B}_{H_{\infty}^{0}}\left(\infty, R_{1}\right) \times H_{\infty}^{+} \times H_{\infty}^{-}
\end{aligned}
$$

satisfy: $z_{n} \rightarrow z_{0}$ and

$$
\begin{aligned}
& u_{n}:=\psi_{1}\left(z_{n}, u_{n}^{\prime}+v_{n}^{\prime}\right) \rightarrow u_{0}=\psi_{1}\left(z_{0}, u_{0}^{\prime}+v_{0}^{\prime}\right), \\
& v_{n}:=\psi_{2}\left(z_{n}, u_{n}^{\prime}+v_{n}^{\prime}\right) \rightarrow v_{0}=\psi_{2}\left(z_{0}, u_{0}^{\prime}+v_{0}^{\prime}\right) .
\end{aligned}
$$

Our goal is to prove that $u_{n}^{\prime} \rightarrow u_{0}^{\prime}$ and $v_{n}^{\prime} \rightarrow v_{0}^{\prime}$.

Step 1. Prove that $\left\{u_{n}^{\prime}\right\}$ and $\left\{v_{n}^{\prime}\right\}$ are bounded.

For each $n$ either $u_{n}^{\prime}=\theta$ or $u_{n}^{\prime} \neq \theta$ and

$$
u_{n}=\frac{\sqrt{F^{\infty}\left(z_{n}, u_{n}^{\prime}+\varphi_{z_{n}}\left(u_{n}^{\prime}\right)\right)}}{\left\|u_{n}^{\prime}\right\|} u_{n}^{\prime}
$$

and hence

$$
\left\|u_{n}\right\|^{2}=F^{\infty}\left(z_{n}, u_{n}^{\prime}+\varphi_{z_{n}}\left(u_{n}^{\prime}\right)\right) \geq F^{\infty}\left(z_{n}, u_{n}^{\prime}\right) \geq \frac{a_{1}}{4}\left\|u_{n}^{\prime}\right\|^{2}
$$

by (2.20) and (2.21). Since $\left\|u_{n}\right\| \rightarrow\left\|u_{0}\right\|$ we deduce that $\left\{u_{n}^{\prime}\right\}$ is bounded and that $u_{n}^{\prime} \rightarrow \theta=u_{0}^{\prime}$ as $n \rightarrow \infty$ if $u_{0}^{\prime}=\theta$ (and so $u_{0}=\theta$ by the definition of $\psi_{1}$ ).

For each $n$, either $v_{n}^{\prime}=\varphi_{z_{n}}\left(u_{n}^{\prime}\right)$ or $v_{n}^{\prime} \neq \varphi_{z_{n}}\left(u_{n}^{\prime}\right)$ and

$$
v_{n}=\frac{\sqrt{F^{\infty}\left(z_{n}, u_{n}^{\prime}+\varphi_{z_{n}}\left(u_{n}^{\prime}\right)\right)-F^{\infty}\left(z_{n}, u_{n}^{\prime}+v_{n}^{\prime}\right)}}{\left\|v_{n}^{\prime}-\varphi_{z_{n}}\left(u_{n}^{\prime}\right)\right\|}\left(v_{n}^{\prime}-\varphi_{z_{n}}\left(u_{n}^{\prime}\right)\right) .
$$

In the latter case $F^{\infty}\left(z_{n}, u_{n}^{\prime}+\varphi_{z_{n}}\left(u_{n}^{\prime}\right)\right)-F^{\infty}\left(z_{n}, u_{n}^{\prime}+v_{n}^{\prime}\right)=\left\|v_{n}\right\|^{2}$. Since $\left\{u_{n}^{\prime}\right\},\left\{z_{n}\right\}$ and thus $\left\{h^{\infty}\left(z_{n}\right)\right\}$ are bounded, it follows from Lemma 2.8(b) that $\left\{\varphi_{z_{n}}\left(u_{n}^{\prime}\right)\right\}$ is bounded, which implies by Lemma 2.7 (b) that $\left\{F^{\infty}\left(z_{n}, u_{n}^{\prime}+\right.\right.$ $\left.\left.\varphi_{z_{n}}\left(u_{n}^{\prime}\right)\right)\right\}$ is bounded. Hence $\left\{F^{\infty}\left(z_{n}, u_{n}^{\prime}+v_{n}^{\prime}\right) \mid v_{n}^{\prime} \neq \varphi_{z_{n}}\left(u_{n}^{\prime}\right)\right\}$ is bounded. Using Lemma 2.7(b) again we deduce that $\left\{v_{n}^{\prime} \mid v_{n}^{\prime} \neq \varphi_{z_{n}}\left(u_{n}^{\prime}\right)\right\}$ is bounded. The claim is proved.

Step 2. Prove that $u_{n}^{\prime} \rightarrow u_{0}^{\prime}$ and $v_{n}^{\prime} \rightarrow v_{0}^{\prime}$.

The first claim has been proved if $u_{0}^{\prime}=\theta$. Let us consider the case $u_{0}^{\prime} \neq \theta$. Since $\left\|\psi_{1}\left(z_{0}, u_{0}^{\prime}+v_{0}^{\prime}\right)\right\|=\sqrt{j\left(z_{0}, u_{0}^{\prime}\right)}>0, \psi_{1}\left(z_{n}, u_{n}^{\prime}+v_{n}^{\prime}\right) \rightarrow \psi_{1}\left(z_{0}, u_{0}^{\prime}+v_{0}^{\prime}\right)$ and 
hence $\left\|\psi_{1}\left(z_{n}, u_{n}^{\prime}+v_{n}^{\prime}\right)\right\|>0$ for large $n$, we deduce that for large $n, u_{n}^{\prime} \neq \theta$ and $j\left(z_{n}, u_{n}^{\prime}\right)=\left\|\psi_{1}\left(z_{n}, u_{n}^{\prime}+v_{n}^{\prime}\right)\right\|^{2}$ converges to $j\left(z_{0}, u_{0}^{\prime}\right)$. Now

$$
\frac{\sqrt{j\left(z_{n}, u_{n}^{\prime}\right)}}{\left\|u_{n}^{\prime}\right\|} u_{n}^{\prime}=u_{n} \rightarrow u_{0}=\frac{\sqrt{j\left(z_{0}, u_{0}^{\prime}\right)}}{\left\|u_{0}^{\prime}\right\|} u_{0}^{\prime} \Rightarrow \frac{u_{n}^{\prime}}{\left\|u_{n}^{\prime}\right\|} \rightarrow \frac{u_{0}^{\prime}}{\left\|u_{0}^{\prime}\right\|} .
$$

Suppose by a contradiction that $\left\{u_{n}^{\prime}\right\}$ does not converge to $u_{0}^{\prime}$. There exists a subsequence $\left\{u_{n_{k}}^{\prime}\right\}$ and $\epsilon>0$ such that $\left\|u_{n_{k}}^{\prime}-u_{0}^{\prime}\right\| \geq \varepsilon$ for all $k$. We may assume that $\left\|u_{n_{k}}^{\prime}\right\| \rightarrow \alpha$ due to the boundedness of $\left\{u_{n}^{\prime}\right\}$. Then $\left\{u_{n_{k}}^{\prime}\right\}$ converges to $\left(\alpha /\left\|u_{0}^{\prime}\right\|\right) u_{0}^{\prime}$ and hence $j\left(z_{n_{k}}, u_{n_{k}}^{\prime}\right) \rightarrow j\left(z_{0},\left(\alpha /\left\|u_{0}^{\prime}\right\|\right) u_{0}^{\prime}\right)=j\left(z_{0}, u_{0}^{\prime}\right)>0$. The latter implies

$$
\psi_{1}\left(z_{0}, \frac{\alpha}{\left\|u_{0}^{\prime}\right\|} u_{0}^{\prime}\right)=\psi_{1}\left(z_{0}, u_{0}^{\prime}\right) .
$$

Since $\left\{v_{n}^{\prime}\right\}$ is bounded, we may assume that $v_{n_{k}}^{\prime} \rightarrow v^{\prime}$ by replaceing $\left\{u_{n_{k}}^{\prime}\right\}$ with a subsequence. Then

$$
\psi_{2}\left(z_{0}, \frac{\alpha}{\left\|u_{0}^{\prime}\right\|} u_{0}^{\prime}+v^{\prime}\right) \leftarrow \psi_{2}\left(z_{n_{k}}, u_{n_{k}}^{\prime}+v_{n_{k}}^{\prime}\right)=v_{n_{k}} \rightarrow v_{0}=\psi_{2}\left(z_{0}, u_{0}^{\prime}+v_{0}^{\prime}\right) .
$$

Obverse that $\psi_{1}$ is independent of elements in $H_{\infty}^{-}$. By (2.29) we get

$$
\psi_{1}\left(z_{0}, \frac{\alpha}{\left\|u_{0}^{\prime}\right\|} u_{0}^{\prime}+v^{\prime}\right)=\psi_{1}\left(z_{0}, u_{0}^{\prime}+v_{0}^{\prime}\right)
$$

and hence

$$
\psi\left(z_{0}, \frac{\alpha}{\left\|u_{0}^{\prime}\right\|} u_{0}^{\prime}+v^{\prime}\right)=\psi\left(z_{0}, u_{0}^{\prime}+v_{0}^{\prime}\right)
$$

The latter implies that $\left(\alpha /\left\|u_{0}^{\prime}\right\|\right) u_{0}^{\prime}=u_{0}^{\prime}$ and $v^{\prime}=v_{0}^{\prime}$ because $\psi\left(z_{0}, \cdot\right)$ is oneto-one. It follows that $\alpha=\left\|u_{0}^{\prime}\right\|$ and $u_{n_{k}}^{\prime} \rightarrow u_{0}^{\prime}$. This contradiction shows that $u_{n}^{\prime} \rightarrow u_{0}^{\prime}$.

Similarly, suppose by a contradiction that $\left\{v_{n}^{\prime}\right\}$ does not converge to $v_{0}^{\prime}$. There exists a subsequence $\left\{v_{n_{k}}^{\prime}\right\}$ and $\varepsilon>0$ such that $\left\|v_{n_{k}}^{\prime}-v_{0}^{\prime}\right\| \geq \varepsilon$ for all $k$. Passing to a subsequence we may assume $v_{n_{k}}^{\prime} \rightarrow v^{\prime}$ as above. Then we also obtain a contradiction because

$$
\psi_{2}\left(z_{0}, u_{0}^{\prime}+v^{\prime}\right) \leftarrow \psi_{2}\left(z_{n_{k}}, u_{n_{k}}^{\prime}+v_{n_{k}}^{\prime}\right)=v_{n_{k}} \rightarrow v_{0}=\psi_{2}\left(z_{0}, u_{0}^{\prime}+v_{0}^{\prime}\right)
$$

and hence $\psi\left(z_{0}, u_{0}^{\prime}+v^{\prime}\right)=\psi\left(z_{0}, u_{0}^{\prime}+v_{0}^{\prime}\right)$ by $(2.29)$, which implies $v^{\prime}=v_{0}^{\prime}$. It contradicts the assumption that $\left\|v^{\prime}-v_{0}^{\prime}\right\| \geq \varepsilon$.

Lemma 2.14. For any $r \in(0, \infty)$ there exists a number $\delta_{r}>0$ such that

$$
\bar{B}_{H_{\infty}^{0}}\left(\infty, R_{1}\right) \times\left(\bar{B}_{H_{\infty}^{+}}\left(\theta, \delta_{r}\right) \oplus \bar{B}_{H_{\infty}^{-}}\left(\theta, \delta_{r}\right)\right)
$$

is contained in

$$
U\left(R_{1}, r\right):=\psi^{-1}\left(B_{H_{\infty}^{+}}\left(\theta, \sqrt{a_{1}} \varepsilon_{r}\right) \oplus B_{H_{\infty}^{-}}\left(\theta, \sqrt{a_{1}} \varepsilon_{r}\right)\right) .
$$

By Lemma 2.11 and Lemma 2.12(a) we have a bijection

$$
\bar{B}_{H_{\infty}^{0}}\left(\infty, R_{1}\right) \times\left(B_{H_{\infty}^{+}}\left(\theta, \sqrt{a_{1}} \varepsilon_{r}\right) \oplus B_{H_{\infty}^{-}}\left(\theta, \sqrt{a_{1}} \varepsilon_{r}\right)\right) \rightarrow U\left(R_{1}, r\right),
$$




$$
(z, u+v) \mapsto(z, \psi(z, u+v)),
$$

whose inverse, denoted by $\phi$, has a form

$$
\phi(z, u+v)=\left(z, \phi_{z}(u+v)\right):=\left(z, u^{\prime}+v^{\prime}\right),
$$

where $\left(u^{\prime}, v^{\prime}\right) \in B_{H_{\infty}^{+}}\left(\theta, \sqrt{a_{1}} \varepsilon_{r}\right) \times B_{H_{\infty}^{-}}\left(\theta, \sqrt{a_{1}} \varepsilon_{r}\right)$ is a unique point satisfying $u+v=\psi\left(z, u^{\prime}+v^{\prime}\right)$. This bijection $\phi$ is actually a homeomorphism and

$$
F^{\infty}(\phi(z, u+v))=\|u\|^{2}-\|v\|^{2} \quad \text { for any }(z, u+v) \in U\left(R_{1}, r\right) .
$$

Moreover, $\phi(z, u+v) \in \operatorname{Im}(\psi) \cap\left(\bar{B}_{H_{\infty}^{0}}\left(\infty, R_{1}\right) \times H_{\infty}^{-}\right)$if and only if $u=\theta$.

Proof. We only prove the first claim. The proofs of others are the same as those of Lemma 2.13.

Let $r \in(0, \infty)$ be given. Since $\psi$ is continuous and $\psi(z, \theta)=\theta$ for any $z \in \bar{B}_{H_{\infty}^{0}}\left(\infty, R_{1}\right)$, it is easily seen that for a given large $R>R_{1}$ we have

$$
\left(\bar{B}_{H_{\infty}^{0}}\left(\infty, R_{1}\right) \cap B_{H_{\infty}^{0}}(\theta, R)\right) \times\left(B_{H_{\infty}^{+}}(\theta, \delta) \oplus B_{H_{\infty}^{-}}(\theta, \delta)\right) \subset U\left(R_{1}, r\right)
$$

for sufficiently small $\delta>0$. So if the conclusion in Lemma 2.14 does not hold for this $r$ then there exist sequences $\left\{z_{n}\right\} \subset \bar{B}_{H_{\infty}^{0}}\left(\infty, R_{1}\right)$ and $\left\{u_{n}^{+}+u_{n}^{-}\right\} \subset H_{\infty}^{ \pm} \backslash\{\theta\}$ such that $\left\|z_{n}\right\| \rightarrow \infty,\left\|u_{n}^{+}+u_{n}^{-}\right\| \rightarrow 0$ (hence $\left\|u_{n}^{+}\right\| \rightarrow 0$ and $\left\|u_{n}^{-}\right\| \rightarrow 0$ ) and

$$
\psi\left(z_{n}, u_{n}^{+}+u_{n}^{-}\right) \notin B_{H_{\infty}^{+}}\left(\theta, \sqrt{a_{1}} \varepsilon_{r}\right) \oplus B_{H_{\infty}^{-}}\left(\theta, \sqrt{a_{1}} \varepsilon_{r}\right) \quad \text { for all } n=1,2, \ldots
$$

The last relation implies that

$$
\text { either }\left\|\psi_{1}\left(z_{n}, u_{n}^{+}+u_{n}^{-}\right)\right\| \geq \sqrt{a_{1}} \varepsilon_{r} \quad \text { or } \quad\left\|\psi_{2}\left(z_{n}, u_{n}^{+}+u_{n}^{-}\right)\right\| \geq \sqrt{a_{1}} \varepsilon_{r}
$$

for each $n=1,2, \ldots$ After passing to a subsequence two cases happen:

- $\left\|\psi_{1}\left(z_{n}, u_{n}^{+}+u_{n}^{-}\right)\right\| \geq \sqrt{a_{1}} \varepsilon_{r}$ for all $n=1,2, \ldots$

- $\left\|\psi_{2}\left(z_{n}, u_{n}^{+}+u_{n}^{-}\right)\right\| \geq \sqrt{a_{1}} \varepsilon_{r}$ for all $n=1,2, \ldots$

In the first case, by the definition of $\psi_{1}$ we have $u_{n}^{+} \neq \theta$ and

$$
F^{\infty}\left(z_{n}, u_{n}^{+}+\varphi_{z_{n}}\left(u_{n}^{+}\right)\right) \geq a_{1} \varepsilon_{r}^{2} \text { for all } n=1,2, \ldots
$$

Since $\left\|u_{n}^{+}\right\| \rightarrow 0$, we may assume that $u_{n}^{+} \in B_{H_{\infty}^{+}}\left(\theta, \varepsilon_{r}\right)$ and hence $\varphi_{z_{n}}\left(u_{n}^{+}\right) \in$ $B_{H_{\infty}^{-}}(\theta, r)$ for all $n \in \mathbb{N}$. After passing to a subsequence we may assume $\varphi_{z_{n}}\left(u_{n}^{+}\right) \rightarrow v_{0} \in H_{\infty}^{-}$. Then Lemma 2.7(a) leads to

$$
F^{\infty}\left(z_{n}, u_{n}^{+}+\varphi_{z_{n}}\left(u_{n}^{+}\right)\right) \rightarrow \frac{1}{2}\left(B(\infty) v_{0}, v_{0}\right)_{H} \leq 0
$$

and hence a contradiction.

In the second case we have $u_{n}^{-} \neq \varphi_{z_{n}}\left(u_{n}^{+}\right)$and

$$
F^{\infty}\left(z_{n}, u_{n}^{+}+\varphi_{z_{n}}\left(u_{n}^{+}\right)\right)-F^{\infty}\left(z_{n}, u_{n}^{+}+u_{n}^{-}\right) \geq a_{1} \varepsilon_{r}^{2} \quad \text { for all } n=1,2, \ldots
$$

As above we may assume $\varphi_{z_{n}}\left(u_{n}^{+}\right) \rightarrow v_{0} \in H_{\infty}^{-}$and use Lemma 2.7(a) to obtain

$$
F^{\infty}\left(z_{n}, u_{n}^{+}+\varphi_{z_{n}}\left(u_{n}^{+}\right)\right)-F^{\infty}\left(z_{n}, u_{n}^{+}+u_{n}^{-}\right) \rightarrow \frac{1}{2}\left(B(\infty) v_{0}, v_{0}\right)_{H} \leq 0 .
$$


This also gives a contradiction. Lemma 2.14 is proved.

Note: If Lemmas 2.4-2.6 only hold in a set $\bar{B}_{H_{\infty}^{0}}\left(\infty, R^{\prime}\right) \oplus\left(\bar{B}_{H}\left(\theta, r^{\prime}\right) \cap X_{\infty}^{ \pm}\right)$, we require $z$ and $r$ in Lemma 2.14 to sit in $\bar{B}_{H_{\infty}^{0}}\left(\infty, R^{\prime}\right)$ and $\left(0, r^{\prime}\right)$, respectively.

Completion of proof of Theorem 1.1. For the homeomorphism in Lemma 2.13 ,

$$
\begin{gathered}
\phi: \bar{B}_{H_{\infty}^{0}}\left(\infty, R_{1}\right) \times\left(H_{\infty}^{+} \oplus H_{\infty}^{-}\right) \rightarrow \bar{B}_{H_{\infty}^{0}}\left(\infty, R_{1}\right) \times\left(H_{\infty}^{+} \oplus H_{\infty}^{-}\right), \\
\left(z, u^{+}+u^{-}\right) \mapsto\left(z, \phi_{z}\left(u^{+}+u^{-}\right)\right),
\end{gathered}
$$

by (2.10) we have

$$
\begin{aligned}
\mathcal{L}\left(z+h^{\infty}(z)+\phi_{z}\left(u^{+}+u^{-}\right)\right)-\mathcal{L}\left(z+h^{\infty}(z)\right) & \\
& =F^{\infty}\left(\phi\left(z, u^{+}+u^{-}\right)\right)=\left\|u^{+}\right\|^{2}-\left\|u^{-}\right\|^{2}
\end{aligned}
$$

for any $\left(z, u^{+}, u^{-}\right) \in \bar{B}_{H_{\infty}^{0}}\left(\infty, R_{1}\right) \times H_{\infty}^{+} \times H_{\infty}^{-}$. Define

$$
\begin{gathered}
\Phi: \bar{B}_{H_{\infty}^{0}}\left(\infty, R_{1}\right) \times\left(H_{\infty}^{+} \oplus H_{\infty}^{-}\right) \rightarrow H, \\
\left(z, u^{+}+u^{-}\right) \mapsto z+h^{\infty}(z)+\phi_{z}\left(u^{+}+u^{-}\right) .
\end{gathered}
$$

Since $h^{\infty}$ takes values in $H_{\infty}^{ \pm}$, it is easy to check that $\Phi$ is a homeomorphism from $\bar{B}_{H_{\infty}^{0}}\left(\infty, R_{1}\right) \times\left(H_{\infty}^{+} \oplus H_{\infty}^{-}\right)$onto $\bar{B}_{H_{\infty}^{0}}\left(\infty, R_{1}\right) \times\left(H_{\infty}^{+} \oplus H_{\infty}^{-}\right)$(by Lemma 2.13), and that

$$
\mathcal{L}\left(\Phi\left(z, u^{+}+u^{-}\right)\right)=\left\|u^{+}\right\|^{2}-\left\|u^{-}\right\|^{2}+\mathcal{L}\left(z+h^{\infty}(z)\right)
$$

for any $\left(z, u^{+}, u^{-}\right) \in \bar{B}_{H_{\infty}^{0}}(\infty, R) \times H_{\infty}^{+} \times H_{\infty}^{-}$. The other conclusions in Theorems 1.1 directly follow from Lemmas $2.2,2.3,2.7,2.8(\mathrm{~b}), 2.10-2.12(\mathrm{~b})$ and Lemma 2.13.

Completion of proof of Theorem 1.3. For the homeomorphism in Lemma 2.14

$$
\begin{gathered}
\phi: U\left(R_{1}, r\right) \rightarrow \bar{B}_{H_{\infty}^{0}}\left(\infty, R_{1}\right) \times\left(B_{H_{\infty}^{+}}\left(\theta, \sqrt{a_{1}} \varepsilon_{r}\right)+B_{H_{\infty}^{-}}\left(\theta, \sqrt{a_{1}} \varepsilon_{r}\right)\right), \\
(z, u+v) \mapsto\left(z, \phi_{z}(u+v)\right),
\end{gathered}
$$

as above we may use (2.10) to get

$$
\mathcal{L}\left(z+h^{\infty}(z)+\phi_{z}\left(u^{+}+u^{-}\right)\right)-\mathcal{L}\left(z+h^{\infty}(z)\right)=\left\|u^{+}\right\|^{2}-\left\|u^{-}\right\|^{2}
$$

for any $\left(z, u^{+}+u^{-}\right) \in U\left(R_{1}, r\right)$. By Lemmas 2.14 and 2.12(a) we have

$$
\begin{aligned}
\overline{C_{R_{1}, \delta_{r}}} & =\bar{B}_{H_{\infty}^{0}}\left(\infty, R_{1}\right) \times\left(\bar{B}_{H_{\infty}^{+}}\left(\theta, \delta_{r}\right) \oplus \bar{B}_{H_{\infty}^{-}}\left(\theta, \delta_{r}\right)\right) \\
& \subset U\left(R_{1}, r\right)=\psi^{-1}\left(B_{H_{\infty}^{+}}\left(\theta, \sqrt{a_{1}} \varepsilon_{r}\right) \oplus B_{H_{\infty}^{-}}\left(\theta, \sqrt{a_{1}} \varepsilon_{r}\right)\right) \\
& \subset \bar{B}_{H_{\infty}^{0}}\left(\infty, R_{1}\right) \times\left(B_{H_{\infty}^{+}}\left(\theta, 2 \varepsilon_{r}\right) \oplus B_{H_{\infty}^{-}}(\theta, r)\right) \subset \overline{C_{R_{1}, r}}
\end{aligned}
$$

(because we may assume $2 \varepsilon_{r}<r$ ). Define

$$
\Phi: C_{R_{1}, \delta_{r}} \rightarrow H, \quad\left(z, u^{+}+u^{-}\right) \mapsto z+h^{\infty}(z)+\phi_{z}\left(u^{+}+u^{-}\right),
$$


and $V(R, r):=\Phi\left(C_{R, \delta_{r}}\right)$ for every $R \geq R_{1}$. Note that $h^{\infty}$ is a map from $\bar{B}_{H_{\infty}^{0}}\left(\infty, R_{1}\right)$ to $\bar{B}_{X_{\infty}^{ \pm}}\left(\theta, \rho_{A}\right)$ by Lemma 2.2 . One easily prove that

$$
V\left(R_{1}, r\right)=\Phi\left(C_{R_{1}, \delta_{r}}\right) \subset \overline{C_{R_{1}, r+\rho_{A}}} .
$$

By Lemma 2.14, (as in the proof of [13, Lemma 2.18] or [14, Lemma 3.6]) one may prove:

(i) $V\left(R_{1}, r\right)$ is an open set of $H$,

(ii) $\Phi$ is a homeomorphism from $C_{R_{1}, \delta_{r}}$ onto $V\left(R_{1}, r\right)$,

(iii) for any $\left(z, u^{+}, u^{-}\right) \equiv z+u^{+}+u^{-} \in C_{R_{1}, \delta_{r}}$,

$$
\mathcal{L}\left(\Phi\left(z, u^{+}+u^{-}\right)\right)=\left\|u^{+}\right\|^{2}-\left\|u^{-}\right\|^{2}+\mathcal{L}\left(z+h^{\infty}(z)\right) .
$$

The other conclusions in Theorem 1.3 follow from Lemmas 2.2, 2.3, 2.7, 2.8(a), 2.10-2.12(a) and Lemma 2.14.

By the Note in Remark 2.9 and the Notes under Lemmas 2.5, 2.8, 2.11, 2.13 and 2.14 one may obtain the conclusions in Remark 1.4. Similarly, that of Remark 1.2 can be obtained.

REMARK 2.15. (a) Under the assumptions that

$$
\mathcal{L} \text { is } C^{2} \text { and } D^{2} \mathcal{L}(w)=B(\infty)+o(1) \text { as }\|w\| \rightarrow \infty,
$$

by increasing $R_{1}$ we may assure that the map

$$
\bar{B}_{H_{\infty}^{0}}\left(\infty, R_{2}\right) \times B_{H_{\infty}^{+}}\left(\theta, r_{\mathcal{L}}\right) \rightarrow H_{\infty}^{-},(z, u) \mapsto \varphi_{z}(u)
$$

is $C^{1}$. In particular, if (1.5) holds then $(z, u) \mapsto \varphi_{z}(u)$ gives a $C^{1}$ map from $\bar{B}_{H_{\infty}^{0}}\left(\infty, R_{1}\right) \times H_{\infty}^{+}$to $H_{\infty}^{-}$. As a consequence, the map $j$ in $(2.19)$ is $C^{1}$ on $\bar{B}_{H_{\infty}^{0}}\left(\infty, R_{1}\right) \times B_{H_{\infty}^{+}}\left(\theta, r_{\mathcal{L}}\right)$.

In fact, since $\mathcal{L}$ is $C^{2}, h^{\infty}$ is $C^{1}$ by the final claim of Lemma 2.2. Moreover, by Remark $2.9 \varphi_{z}(u) \in H_{\infty}^{-}$is the unique maximum point of the function

$$
H_{\infty}^{-} \rightarrow \mathbb{R}, v \mapsto F^{\infty}(z, u+v)=\mathcal{L}\left(z+h^{\infty}(z)+u+v\right)-\mathcal{L}\left(z+h^{\infty}(z)\right) .
$$

We derive $\left(\nabla \mathcal{L}\left(z+h^{\infty}(z)+u+\varphi_{z}(u)\right), v\right)_{H}=0 \forall v \in H_{\infty}^{-}$, that is,

$$
P_{\infty}^{-} \nabla \mathcal{L}\left(z+h^{\infty}(z)+u+\varphi_{z}(u)\right)=\theta .
$$

Consider the map $\Xi: \bar{B}_{H_{\infty}^{0}}\left(\infty, R_{1}\right) \times B_{H_{\infty}^{+}}\left(\theta, r_{\mathcal{L}}\right) \times H_{\infty}^{-} \rightarrow H_{\infty}^{-}$given by

$$
\Xi(z, u, v)=P_{\infty}^{-} \nabla \mathcal{L}\left(z+h^{\infty}(z)+u+v\right) .
$$

It is $C^{1}$ and

$$
D_{v} \Xi\left(z, u, \varphi_{z}(u)\right)=\left.P_{\infty}^{-} D^{2} \mathcal{L}\left(z+h^{\infty}(z)+u+\varphi_{z}(u)\right)\right|_{H_{\infty}^{-}}: H_{\infty}^{-} \rightarrow H_{\infty}^{-} .
$$

Since $\left\|z+h^{\infty}(z)+u+\varphi_{z}(u)\right\|^{2}=\|z\|^{2}+\left\|h^{\infty}(z)+u+\varphi_{z}(u)\right\|^{2} \geq\|z\|^{2}$ and $D^{2} \mathcal{L}(w)=B(\infty)+o(1)$ as $\|w\| \rightarrow \infty$ we can increase $R_{1}$ so that for any $(z, u) \in$ $\bar{B}_{H_{\infty}^{0}}\left(\infty, R_{1}\right) \times B_{H_{\infty}^{+}}\left(\theta, r_{\mathcal{L}}\right)$ the operator $D_{v} \Xi\left(z, u, \varphi_{z}(u)\right)$ has a bounded inverse. Hence the desired conclusion follows from the implicit function theorem. 
(b) Under the assumption (2.31), the homeomorphism

$$
\begin{gathered}
\phi^{-1}: \bar{B}_{H_{\infty}^{0}}\left(\infty, R_{2}\right) \times\left(B_{H_{\infty}^{+}}\left(\theta, \sqrt{a_{1}} \varepsilon_{r}\right)+B_{H_{\infty}^{-}}\left(\theta, \sqrt{a_{1}} \varepsilon_{r}\right)\right) \rightarrow U\left(R_{2}, r\right), \\
(z, u+v) \mapsto(z, \psi(z, u+v)),
\end{gathered}
$$

is $C^{1}$ on $\bar{B}_{H_{\infty}^{0}}\left(\infty, R_{1}\right) \times\left(B_{H_{\infty}^{+}}\left(\theta, \sqrt{a_{1}} \varepsilon_{r}\right)+B_{H_{\infty}^{-}}\left(\theta, \sqrt{a_{1}} \varepsilon_{r}\right)\right) \backslash \triangle_{r}$, where

$$
\triangle_{r}:=\left\{\left(z, u+\varphi_{z}(u)\right) \mid(z, u) \in \bar{B}_{H_{\infty}^{0}}\left(\infty, R_{1}\right) \times B_{H_{\infty}^{+}}\left(\theta, r_{\mathcal{L}}\right)\right\}
$$

is a $C^{1}$-submanifold of $\bar{B}_{H_{\infty}^{0}}\left(\infty, R_{1}\right) \times H_{\infty}^{ \pm}$of codimension $\mu_{\infty}$.

Indeed, it has been proved that the map $j$ in $(2.19)$ is $C^{1}$ on $\bar{B}_{H_{\infty}^{0}}\left(\infty, R_{1}\right) \times$ $B_{H_{\infty}^{+}}\left(\theta, r_{\mathcal{L}}\right)$ above. Then the construction of $\psi$ directly gives the desired conclusion.

Let $V\left(R_{1}, r\right)$ be as in the proof of Theorem 1.3. Write a point of $V\left(R_{1}, r\right)$ as $\left(z, u^{+}+u^{-}\right)$, where $z \in B_{H_{\infty}^{0}}\left(\infty, R_{1}\right)$ and $u^{*} \in H_{\infty}^{*}, *=+,-$. It is easily checked that $\Phi^{-1}: V\left(R_{1}, r\right) \rightarrow C_{R_{1}, \delta_{r}}$ is given by

$$
\Phi^{-1}\left(z, u^{+}+u^{-}\right)=\phi^{-1}\left(z, u^{+}+u^{-}-h^{\infty}(z)\right)=\left(z, \psi\left(z, u^{+}+u^{-}-h^{\infty}(z)\right)\right) .
$$

Note that $h^{\infty}$ is $C^{1}$ (because $\mathcal{L}$ is $C^{2}$ ). Hence $\Phi^{-1}$ is $C^{1}$ outside the submanifold of codimension $\mu_{\infty}$,

$$
\widetilde{\triangle}_{r}:=\left\{\left(z, u+\varphi_{z}(u)+h^{\infty}(z)\right) \mid(z, u) \in \bar{B}_{H_{\infty}^{0}}\left(\infty, R_{1}\right) \times B_{H_{\infty}^{+}}\left(\theta, r_{\mathcal{L}}\right)\right\} .
$$

Furthermore, if (1.5) holds, the restriction of $\phi^{-1}$ to $\bar{B}_{H_{\infty}^{0}}\left(\infty, R_{1}\right) \times\left(H_{\infty}^{+} \oplus H_{\infty}^{-}\right)$,

$$
\begin{gathered}
\bar{B}_{H_{\infty}^{0}}\left(\infty, R_{1}\right) \times\left(H_{\infty}^{+} \oplus H_{\infty}^{-}\right) \rightarrow \bar{B}_{H_{\infty}^{0}}\left(\infty, R_{1}\right) \times\left(H_{\infty}^{+} \oplus H_{\infty}^{-}\right), \\
(z, u+v) \mapsto(z, \psi(z, u+v)),
\end{gathered}
$$

is $C^{1}$ outside $\triangle_{\infty}:=\left\{\left(z, u+\varphi_{z}(u)\right) \mid(z, u) \in \bar{B}_{H_{\infty}^{0}}\left(\infty, R_{1}\right) \times H_{\infty}^{+}\right\}$. Since

$$
\Phi^{-1}: B_{H_{\infty}^{0}}\left(\infty, R_{1}\right) \times\left(H_{\infty}^{+} \oplus H_{\infty}^{-}\right) \rightarrow B_{H_{\infty}^{0}}\left(\infty, R_{1}\right) \times\left(H_{\infty}^{+} \oplus H_{\infty}^{-}\right)
$$

is given by

$$
\Phi^{-1}\left(z, u^{+}+u^{-}\right)=\phi^{-1}\left(z, u^{+}+u^{-}-h^{\infty}(z)\right)=\left(z, \psi\left(z, u^{+}+u^{-}-h^{\infty}(z)\right)\right),
$$

we see that $\Phi^{-1}$ is $C^{1}$ outside the submanifold of codimension $\mu_{\infty}$,

$$
\widetilde{\triangle}_{\infty}:=\left\{\left(z, u+\varphi_{z}(u)+h^{\infty}(z)\right) \mid(z, u) \in \bar{B}_{H_{\infty}^{0}}\left(\infty, R_{1}\right) \times H_{\infty}^{+}\right\} .
$$

\subsection{The proof of Theorem 1.8 .}

2.2.1. Case $\mu_{\infty}=0$, i.e. $H_{\infty}^{-}=\{\theta\}$. By (1.1) and (1.12), for any $u \in$ $\bar{B}_{H}(\infty, R) \cap X$ we have

$$
\begin{aligned}
D \mathcal{L}(u) u & =D \mathcal{L}(u) u-(B(\infty) u, u)_{H}+(B(\infty) u, u)_{H} \\
& =(A(u)-B(\infty) u, u)_{H}+(B(\infty) u, u)_{H} \\
& \geq 2 a_{\infty}\|u\|^{2}-\|A(u)-B(\infty) u\| \cdot\|u\| \geq\left(2 a_{\infty}-\lambda\right)\|u\|^{2} \geq a_{\infty}\|u\|^{2} .
\end{aligned}
$$


Since $\mathcal{L}$ is continuously directional differentiable and $X$ is dense in $H$ we get

$$
D \mathcal{L}(u) u \geq a_{\infty}\|u\|^{2} \quad \text { for all } u \in \bar{B}_{H}(\infty, R) .
$$

Define $\psi: \bar{B}_{H}(\infty, R) \rightarrow H$ by $\psi(u)=\sqrt{\mathcal{L}(u)} /\|u\| u$.

Claim. $\psi$ is injective.

In fact, if there exist $u_{1}, u_{2} \in \bar{B}_{H}(\infty, R), u_{1} \neq u_{2}$, such that $\psi\left(u_{1}\right)=\psi\left(u_{2}\right)$. Then $\mathcal{L}\left(u_{1}\right)=\mathcal{L}\left(u_{2}\right)$ and so $u_{1} /\left\|u_{1}\right\|=u_{2} /\left\|u_{2}\right\|$. This implies $\left\|u_{1}\right\| \neq\left\|u_{2}\right\|$. We may assume $\left\|u_{2}\right\|>\left\|u_{1}\right\|$. Then $u_{2}=k u_{1}, k>1$. Obverse that $t u_{1}+(1-t) u_{2}=$ $(t+(1-t) k) u_{1} \in \bar{B}_{H}(\infty, R)$ for all $t \in[0,1]$. We derive

$$
\begin{aligned}
\mathcal{L}\left(u_{2}\right)-\mathcal{L}\left(u_{1}\right) & =\left.\mathcal{L}\left(t u_{2}+(1-t) u_{1}\right)\right|_{t=0} ^{t=1} \\
& =D \mathcal{L}\left(t u_{2}+(1-t) u_{1}\right)\left(u_{2}-u_{1}\right) \\
& =D \mathcal{L}\left([t k+(1-t)] u_{1}\right)\left((k-1) u_{1}\right) \\
& =\frac{k-1}{t k+(1-t)} D \mathcal{L}\left([t k+(1-t)] u_{1}\right)\left((t k+1-t) u_{1}\right) \\
& \geq a_{\infty} \frac{k-1}{t k+(1-t)}\left\|(t k+1-t) u_{1}\right\|^{2} \\
& =a_{\infty}(k-1)(t k+1-t)\left\|u_{1}\right\|^{2}>0
\end{aligned}
$$

because of (2.32). This contradiction shows that $\psi$ is injective.

By (1.11), for any $u \in \bar{B}_{H}(\infty, R)=\bar{B}_{H_{\infty}^{+}}(\infty, R)$ we get

$$
\left(a_{\infty}+\lambda\right)\|u\|^{2} \geq \mathcal{L}(u) \geq\left(a_{\infty}-\lambda\right)\|u\|^{2}
$$

and hence

$$
\sqrt{2 a_{\infty}} \geq \frac{\sqrt{\mathcal{L}(u)}}{\|u\|} \geq \sqrt{a_{\infty}-\lambda} \text { for all } u \in \bar{B}_{H}(\infty, R) .
$$

For $\zeta \in \bar{B}_{H}\left(\infty, \sqrt{2 a_{\infty}} R\right)$ let $\bar{\zeta}=\frac{R}{\|\zeta\|} \zeta$. Take $t_{2}>1$ such that

$$
\sqrt{\mathcal{L}\left(t_{2} \bar{\zeta}\right)} \geq \sqrt{a_{\infty}-\lambda} t_{2}\|\bar{\zeta}\|>\|\zeta\| \geq \sqrt{2 a_{\infty}} R=\sqrt{2 a_{\infty}}\|\bar{\zeta}\| \geq \sqrt{\mathcal{L}(\bar{\zeta})}
$$

Since $t \mapsto \mathcal{L}(t \bar{\zeta})$ is continuous, the intermediate value theorem yields a number $t_{1} \in\left[1, t_{2}\right]$ such that $\|\zeta\|=\sqrt{\mathcal{L}\left(t_{1} \bar{\zeta}\right)}$ and hence

$$
\psi\left(t_{1} \bar{\zeta}\right)=\sqrt{\mathcal{L}\left(t_{1} \bar{\zeta}\right)} \cdot \frac{t_{1} \bar{\zeta}}{\left\|t_{1} \bar{\zeta}\right\|}=\|\zeta\| \cdot \frac{\zeta}{\|\zeta\|}=\zeta
$$

This shows that $\bar{B}_{H}\left(\infty, \sqrt{2 a_{\infty}} R\right) \subset \psi\left(\bar{B}_{H}(\infty, R)\right)$. Hence it follows from the above claim that, for each $u \in \bar{B}_{H}\left(\infty, \sqrt{2 a_{\infty}} R\right)$, there exists a unique $\phi(u) \in$ $\bar{B}_{H}(\infty, R)$ such that $\psi(\phi(u))=u$. Clearly, the map $\phi: \bar{B}_{H}\left(\infty, \sqrt{2 a_{\infty}} R\right) \rightarrow$ $\bar{B}_{H}(\infty, R)$ is injective. By the definition of $\psi$,

$$
u=\psi(\phi(u))=\frac{\sqrt{\mathcal{L}(\phi(u))}}{\|\phi(u)\|} \phi(u) \quad \text { and so } \quad \mathcal{L}(\phi(u))=\|u\|^{2}
$$


for any $u \in \bar{B}_{H}\left(\infty, \sqrt{2 a_{\infty}} R\right)$. Since

we deduce that

$$
\sqrt{2 a_{\infty}} \geq \frac{\sqrt{\mathcal{L}(\phi(u))}}{\|\phi(u)\|} \geq \sqrt{a_{\infty}-\lambda},
$$

$$
\frac{\|u\|}{\sqrt{2 a_{\infty}}} \leq\|\phi(u)\| \leq \frac{1}{\sqrt{a_{\infty}-\lambda}}\|u\| \quad \text { for all } u \in \bar{B}_{H}\left(\infty, \sqrt{2 a_{\infty}} R\right) .
$$

Let $\left\{\zeta_{k}\right\}_{k=1}^{\infty} \subset \bar{B}_{H}\left(\infty, \sqrt{2 a_{\infty}} R\right)$ converge to $\zeta \in \bar{B}_{H}\left(\infty, \sqrt{2 a_{\infty}} R\right)$. Set $\eta_{k}=$ $\phi\left(\zeta_{k}\right)$ and $\eta=\phi(\zeta)$. Then $\psi\left(\eta_{k}\right)=\zeta_{k}$ and $\psi(\eta)=\zeta$. So $\left\|\zeta_{k}\right\| \rightarrow\|\zeta\|$ implies $\mathcal{L}\left(\eta_{k}\right) \rightarrow \mathcal{L}(\eta)$. Note that

$$
\psi\left(\eta_{k}\right)=\frac{\sqrt{\mathcal{L}\left(\eta_{k}\right)}}{\left\|\eta_{k}\right\|} \eta_{k}=\frac{\sqrt{\mathcal{L}\left(\eta_{k}\right)}}{\left\|\zeta_{k}\right\|} \zeta_{k} \quad \text { and } \quad \psi(\eta)=\frac{\sqrt{\mathcal{L}(\eta)}}{\|\eta\|} \eta=\frac{\sqrt{\mathcal{L}(\eta)}}{\|\zeta\|} \zeta .
$$

We deduce that $\eta_{k} \rightarrow \eta$. That is, $\phi$ is continuous. Hence $\phi$ is a homeomorphism onto its image and satisfies: $\mathcal{L}(\phi(u))=\|u\|^{2}$ for all $u \in \bar{B}_{H}\left(\infty, \sqrt{2 a_{\infty}} R\right)$. Taking $\Re=\sqrt{2 a_{\infty}} R$ gives the desired conclusion.

2.2.2. Case $\mu_{\infty}>0$. Note that Lemmas 2.4, 2.5 still hold with $H_{\infty}^{0}=\{\theta\}$ under the conditions $\left(\mathrm{C} 1_{\infty}\right)-\left(\mathrm{C} 2_{\infty}\right)$ and $\left(\mathrm{D}_{\infty}\right)$. Let us give the corresponding result with Lemma 2.6.

Lemma 2.16. Let $R_{1}>0$ be as Lemmas $2.4,2.5$ and $R_{2}=\max \left\{R, R_{1}\right\}$. Then:

(a) $\left[D \mathcal{L}\left(u+v_{2}\right)-D \mathcal{L}\left(u+v_{1}\right)\right]\left(v_{2}-v_{1}\right) \leq-a_{\infty}\left\|v_{2}-v_{1}\right\|^{2}<0$ for any $u \in H_{\infty}^{+}$ with $\|u\| \geq R_{2}$, and $v_{1}, v_{2} \in H_{\infty}^{-}$with $v_{1} \neq v_{2}$;

(b) $D \mathcal{L}(u+v)(u-v) \geq a_{1}\|u\|^{2}+a_{\infty}\|v\|^{2}>0$ for any $(u, v) \in H_{\infty}^{+} \times H_{\infty}^{-}$ with $(u, v) \neq(\theta, \theta)$;

(c) $D \mathcal{L}(u) u \geq a_{\infty}\|u\|^{2}>p(\|u\|)$ for any $u \in H_{\infty}^{+}$with $\|u\| \geq R$, where $p(t)=a_{\infty} t^{2} / 2$.

Proof. (a) For any $u^{+} \in X_{\infty}^{+}$with $\left\|u^{+}\right\| \geq R_{1}$ and $u_{1}^{-}, u_{2}^{-} \in H_{\infty}^{-}$, since the function $X \ni u \mapsto\left(A\left(u^{+}+u\right), u_{2}^{-}-u_{1}^{-}\right)_{H}$ is continuously directional differentiable, by the condition $\left(\mathrm{F} 2{ }_{\infty}\right)$ and the mean value theorem we have a number $t \in(0,1)$ such that

$$
\begin{aligned}
{\left[D \mathcal{L}\left(u^{+}+u_{2}^{-}\right)-D \mathcal{L}\left(u^{+}+u_{1}^{-}\right)\right]\left(u_{2}^{-}-u_{1}^{-}\right) } \\
\quad=\left(A\left(u^{+}+u_{2}^{-}\right), u_{2}^{-}-u_{1}^{-}\right)_{H}-\left(A\left(u^{+}+u_{1}^{-}\right), u_{2}^{-}-u_{1}^{-}\right)_{H} \\
\quad=\left(D A\left(u^{+}+u_{1}^{-}+t\left(u_{2}^{-}-u_{1}^{-}\right)\right)\left(u_{2}^{-}-u_{1}^{-}\right), u_{2}^{-}-u_{1}^{-}\right)_{H} \\
\quad=\left(B\left(u^{+}+u_{1}^{-}+t\left(u_{2}^{-}-u_{1}^{-}\right)\right)\left(u_{2}^{-}-u_{1}^{-}\right), u_{2}^{-}-u_{1}^{-}\right)_{H} \leq-a_{\infty}\left\|u_{2}^{-}-u_{1}^{-}\right\|^{2},
\end{aligned}
$$

where the third equality comes from $\left(\mathrm{F} 3_{\infty}\right)$, and the final inequality is due to the fact that $\left\|u^{+}+u_{1}^{-}+t\left(u_{2}^{-}-u_{1}^{-}\right)\right\| \geq\left\|u^{+}\right\| \geq R_{1}$ and Lemma 2.5(c). Hence the desired conclusion follows from the density of $X_{\infty}^{+}$in $H_{\infty}^{+}$. 
(b) By (1.12), $\|A(u)-B(\infty) u\| \leq \lambda\|u\|$ for any $u \in X$ with $\|u\| \geq R_{2}$. Because $X_{\infty}^{+}$is dense in $H_{\infty}^{+}$, as above it suffices to prove the conclusion for $u^{+} \in X_{\infty}^{+}$with $\left\|u^{+}\right\| \geq R_{2}$, and $u^{-} \in H_{\infty}^{-}$. Note that $\left\|u^{+}+u^{-}\right\| \geq R_{2}$. We have

$$
\begin{aligned}
D \mathcal{L}\left(u^{+}\right. & \left.+u^{-}\right)\left(u^{+}-u^{-}\right)=\left(A\left(u^{+}+u^{-}\right)-B(\infty)\left(u^{+}+u^{-}\right), u^{+}-u^{-}\right)_{H} \\
& +\left(B(\infty)\left(u^{+}+u^{-}\right), u^{+}-u^{-}\right)_{H} \\
\geq & \left(B(\infty) u^{+}, u^{+}\right)-\left(B(\infty) u^{-}, u^{-}\right)_{H} \\
& -\left\|A\left(u^{+}+u^{-}\right)-B(\infty)\left(u^{+}+u^{-}\right)\right\| \cdot\left\|u^{+}-u^{-}\right\| \\
\geq & 2 a_{\infty}\left(\left\|u^{+}\right\|^{2}+\left\|u^{-}\right\|^{2}\right)-\lambda\left\|u^{+}+u^{-}\right\| \cdot\left\|u^{+}-u^{-}\right\| \\
= & 2 a_{\infty}\left(\left\|u^{+}\right\|^{2}+\left\|u^{-}\right\|^{2}\right)-\lambda \sqrt{\left\|u^{+}+u^{-}\right\|^{2}} \cdot \sqrt{\left\|u^{+}-u^{-}\right\|^{2}} \\
= & 2 a_{\infty}\left(\left\|u^{+}\right\|^{2}+\left\|u^{-}\right\|^{2}\right)-\lambda \sqrt{\left\|u^{+}\right\|^{2}+\left\|u^{-}\right\|^{2}} \cdot \sqrt{\left\|u^{+}\right\|^{2}+\left\|u^{-}\right\|^{2}} \\
\geq & a_{\infty}\left(\left\|u^{+}\right\|^{2}+\left\|u^{-}\right\|^{2}\right) .
\end{aligned}
$$

(c) can be proved as that of (2.32).

By (1.11), for any $u^{+}+u^{-} \in \bar{B}_{H}\left(\infty, R_{2}\right)$ we have

$$
\begin{aligned}
\mathcal{L}\left(u^{+}+u^{-}\right) & \leq \frac{1}{2}\left(B(\infty)\left(u^{+}+u^{-}\right), u^{+}+u^{-}\right)+\lambda\left\|u^{+}+u^{-}\right\|^{2} \\
& =\frac{1}{2}\left(B(\infty) u^{+}, u^{+}\right)+\frac{1}{2}\left(B(\infty) u^{-}, u^{-}\right)+\lambda\left\|u^{+}+u^{-}\right\|^{2} \\
& \leq\|B(\infty)\| \cdot\left\|u^{+}\right\|^{2}-a_{\infty}\left\|u^{-}\right\|^{2}+\lambda\left\|u^{+}\right\|^{2}+\lambda\left\|u^{-}\right\|^{2} \\
& \leq 2\|B(\infty)\|\left\|u^{+}\right\|^{2}-\left(a_{\infty}-\lambda\right)\left\|u^{-}\right\|^{2}
\end{aligned}
$$

because (1.1) implies the inequality $a_{\infty} \leq\|B(\infty)\|$. In particular, for any $u^{+} \in H_{\infty}^{+}$it holds that $\mathcal{L}\left(u^{+}+u^{-}\right) \rightarrow-\infty$ as $u^{-} \in H_{\infty}^{-}$and $\left\|u^{-}\right\| \rightarrow \infty$. By Lemma 2.16(a), for each $u^{+} \in H_{\infty}^{+}$with $\left\|u^{+}\right\| \geq R_{2}$ the function $H_{\infty}^{-} \ni$ $u^{-} \mapsto-\mathcal{L}\left(u^{+}+u^{-}\right)$is strictly convex. Hence $H_{\infty}^{-} \ni u^{-} \mapsto \mathcal{L}\left(u^{+}+u^{-}\right)$attains the maximum at a unique point $\varphi\left(u^{+}\right) \in H_{\infty}^{-}$. Define

$$
j: H_{\infty}^{+} \rightarrow \mathbb{R}, u^{+} \mapsto \mathcal{L}\left(u^{+}+\varphi\left(u^{+}\right)\right) .
$$

Then $j\left(u^{+}\right) \rightarrow+\infty$ as $u^{+} \in H_{\infty}^{+}$and $\left\|u^{+}\right\| \rightarrow \infty$ because

(2.34) $\mathcal{L}\left(u^{+}+\varphi\left(u^{+}\right)\right) \geq \mathcal{L}\left(u^{+}\right)=\frac{1}{2}\left(B(\infty) u^{+}, u^{+}\right)-\lambda\left\|u^{+}\right\|^{2} \geq\left(a_{\infty}-\lambda\right)\left\|u^{+}\right\|^{2}$.

As in the proof of Lemma 2.10 we may prove that $j$ is continuous, and continuously directional differentiable. For $(u, v) \in \bar{B}_{H_{\infty}^{+}}\left(\infty, R_{2}\right) \times H_{\infty}^{-}$define

$$
\begin{aligned}
& \psi_{1}(u+v)=\frac{\sqrt{\mathcal{L}(u+\varphi(u))}}{\|u\|} u, \\
& \psi_{2}(u+v)= \begin{cases}\frac{\sqrt{\mathcal{L}(u+\varphi(u))-\mathcal{L}(u+v)}}{\|v-\varphi(u)\|}(v-\varphi(u)) & \text { if } v \neq \varphi(u), \\
\theta & \text { if } v=\varphi(u) .\end{cases}
\end{aligned}
$$


Then the map

$$
\psi=\psi_{1}+\psi_{2}: \bar{B}_{H_{\infty}^{+}}\left(\infty, R_{2}\right) \oplus H_{\infty}^{-} \rightarrow H_{\infty}^{ \pm}
$$

is continuous, and satisfies: $\mathcal{L}(u+v)=\left\|\psi_{1}(u+v)\right\|^{2}-\left\|\psi_{2}(u+v)\right\|^{2}$.

For $u \in \bar{B}_{H_{\infty}^{+}}\left(\infty, R_{2}\right)$, since $\|u+\varphi(u)\|^{2}=\|u\|^{2}+\|\varphi(u)\|^{2}$, by $(2.33)-(2.34)$ we have

$$
2\|B(\infty)\|\|u\|^{2} \geq \mathcal{L}(u+\varphi(u)) \geq \mathcal{L}(u) \geq\left(a_{\infty}-\lambda\right)\|u\|^{2} .
$$

For $\zeta \in \bar{B}_{H_{\infty}^{+}}\left(\infty, \sqrt{2\|B(\infty)\|} R_{2}\right)$ let $\bar{\zeta}=\left(R_{2} /\|\zeta\|\right) \zeta$. By $(2.36)$ we may take $t_{2}>1$ such that

$$
\begin{aligned}
\sqrt{\mathcal{L}\left(t_{2} \bar{\zeta}+\varphi\left(t_{2} \bar{\zeta}\right)\right)} \geq \sqrt{a_{\infty}-\lambda} \cdot t_{2}\|\bar{\zeta}\| & >\|\zeta\| \geq \sqrt{2\|B(\infty)\|} R_{2} \\
& =\sqrt{2\|B(\infty)\|} \cdot\|\bar{\zeta}\| \geq \sqrt{\mathcal{L}(\bar{\zeta}+\varphi(\bar{\zeta}))}
\end{aligned}
$$

Since $t \mapsto \mathcal{L}(t \bar{\zeta}+\varphi(t \bar{\zeta}))$ is continuous, as above we have a number $t_{1} \in\left[1, t_{2}\right]$ such that $\|\zeta\|=\sqrt{\mathcal{L}\left(t_{1} \bar{\zeta}+\varphi\left(t_{1} \bar{\zeta}\right)\right)}$ and hence

$$
\psi_{1}\left(t_{1} \bar{\zeta}\right)=\sqrt{\mathcal{L}\left(t_{1} \bar{\zeta}+\varphi\left(t_{1} \bar{\zeta}\right)\right)} \cdot \frac{t_{1} \bar{\zeta}}{\left\|t_{1} \bar{\zeta}\right\|}=\|\zeta\| \cdot \frac{\zeta}{\|\zeta\|}=\zeta
$$

Let $\xi \in H_{\infty}^{-}$and $\xi \neq 0$. Note that the function

$$
[0, \infty) \ni s \mapsto \mathcal{L}\left(t_{1} \bar{\zeta}+\varphi\left(t_{1} \bar{\zeta}\right)\right)-\mathcal{L}\left(t_{1} \bar{\zeta}+\varphi\left(t_{1} \bar{\zeta}\right)+s v\right)
$$

takes over all values in $[0, \infty)$ for any $v \in H_{\infty}^{-} \backslash\{\theta\}$. Take $v=\xi$. We have a number $s>0$ such that

$$
\sqrt{\mathcal{L}\left(t_{1} \bar{\zeta}+\varphi\left(t_{1} \bar{\zeta}\right)\right)-\mathcal{L}\left(t_{1} \bar{\zeta}+\varphi\left(t_{1} \bar{\zeta}\right)+s \xi\right)}=\|\xi\|
$$

Set $v:=\varphi\left(t_{1} \bar{\zeta}\right)+s \xi$. Then

$$
\psi_{2}\left(t_{1} \bar{\zeta}+v\right)=\frac{\sqrt{\mathcal{L}\left(t_{1} \bar{\zeta}+\varphi\left(t_{1} \bar{\zeta}\right)\right)-\mathcal{L}\left(t_{1} \bar{\zeta}+v\right)}}{\left\|v-\varphi\left(t_{1} \bar{\zeta}\right)\right\|}\left(v-\varphi\left(t_{1} \bar{\zeta}\right)\right)=\frac{\|\xi\|}{\|s \xi\|} s \xi=\xi
$$

Hence $\psi\left(t_{1} \bar{\zeta}+v\right)=\zeta+\xi$. This shows that

$$
\bar{B}_{H_{\infty}^{+}}\left(\infty, \sqrt{2\|B(\infty)\|} R_{2}\right) \oplus H_{\infty}^{-} \subset \psi\left(\bar{B}_{H}\left(\infty, R_{2}\right)\right)=\psi_{1}\left(\bar{B}_{H}\left(\infty, R_{2}\right)\right) \oplus H_{\infty}^{-} .
$$

As in the proofs of Lemmas 2.11, 2.12(b) and 2.13 we can show that $\psi$ is a homeomorphism onto its image (by increasing $R_{2}>0$ if necessary). Let $\phi$ denote the restriction of $\psi^{-1}$ to $\bar{B}_{H_{\infty}^{+}}\left(\infty, \sqrt{2\|B(\infty)\|} R_{2}\right) \oplus H_{\infty}^{-}$. Set $\mathfrak{R}=$ $\sqrt{2 \| B(\infty)} R_{2}$. We get

$$
\mathcal{L}(\phi(u+v))=\|u\|^{2}-\|v\|^{2} \quad \text { for all }(u, v) \in \bar{B}_{H_{\infty}^{+}}(\infty, \mathfrak{R}) \times H_{\infty}^{-} .
$$




\section{Relations to previous splitting lemmas at infinity}

3.1. Relations to the splitting lemma at infinity in [2]. We begin with the following elementary functional analysis fact.

Lemma 3.1. Let $A_{0}$ be a bounded linear self-adjoint operator on a Hilbert space $H$ and let 0 be an isolated point of $\sigma\left(A_{0}\right)$. Let $H^{0}=N\left(A_{0}\right)=\operatorname{Ker}\left(A_{0}\right)$ and $H^{+}\left(\right.$resp. $\left.H^{-}\right)$be the positive (resp. negative) definite subspace of $A_{0}$. Suppose that both $H^{0}$ and $H^{-}$are finite dimensional and that there exists a number $\alpha>0$ such that $*\left(A u^{*}, u^{*}\right) \geq 2 \alpha\left\|u^{*}\right\|^{2}$ for all $u^{*} \in H^{*}, *=+,-$. Then $A_{0}$ can be expressed as a sum $P+Q$, where $Q \in L_{s}(H)$ is compact and $P \in L_{s}(H)$ satisfies: $(P u, u) \geq 2 \alpha\|u\|^{2}$ for all $u \in H$.

Proof. Since $A_{0}$ is self-adjoint and 0 is an isolated point of $\sigma\left(A_{0}\right)$, by Proposition 4.5 of [7] the range $R\left(A_{0}\right)$ is closed, and hence $N\left(A_{0}\right)^{\perp}=N\left(A_{0}^{*}\right)^{\perp}=$ $\overline{R\left(A_{0}\right)}=R\left(A_{0}\right)$. It follows that $R(A)=H^{+} \oplus H^{-}$and $H=H^{0} \oplus R(A)=$ $H^{0} \oplus H^{-} \oplus H^{+}$. Let $P^{0}: H \rightarrow H^{0} \oplus H^{-}$be the orthogonal projection, which is an operator of finite rank and hence compact. Define operators $P, Q \in L_{s}(H)$ by

$$
\begin{aligned}
& P u=2 \alpha u \quad \text { if } u \in H^{0}, \quad P u=* A_{0} u \quad \text { if } u \in H^{*}, *=+,- \text {, } \\
& Q u=A_{0} u-P u \quad \text { if } u \in H^{0} \oplus H^{-}, \quad Q u=\theta \quad \text { if } u \in H^{+} .
\end{aligned}
$$

Then $A_{0}=P+Q, Q$ is of finite rank and hence compact, and $P$ satisfies

$$
\begin{aligned}
(P u, u)_{H} & =\left(P u^{0}, u^{0}\right)_{H}+\left(P u^{-}, u^{-}\right)_{H}+\left(P u^{+}, u^{+}\right)_{H} \\
& \geq 2 \alpha\left\|u^{0}\right\|^{2}+2 \alpha\left\|u^{-}\right\|^{2}+2 \alpha\left\|u^{+}\right\|^{2}=2 \alpha\|u\|^{2}
\end{aligned}
$$

for any $u=u^{0}+u^{-}+u^{+} \in H^{0} \oplus H^{-} \oplus H^{+}=H$.

Recall the following basic assumption in [2, p. 425]:

$\left(\mathrm{A}_{\infty}\right) f(x)=\left(A_{0} x, x\right)_{H} / 2+g(x)$ where $A_{0}: H \rightarrow H$ is a self-adjoint linear operator such that 0 is isolated in the spectrum of $A_{0}$. The map $g \in C^{1}(H, \mathbb{R})$ is of class $C^{2}$ in a neighbourhood of infinity and satisfies $g^{\prime \prime}(x) \rightarrow 0$ as $\|x\| \rightarrow \infty$. Moreover, $g$ and $g^{\prime}$ map bounded sets to bounded sets.

(Note: It was claimed below $\left(\mathrm{A}_{\infty}\right)$ in [2] that $\left(\mathrm{A}_{\infty}\right)$ implies: $g(x)=o\left(\|x\|^{2}\right)$ and $g^{\prime}(x)=o(\|x\|)$ as $\|x\| \rightarrow \infty$, which are used in the proof of Lemma 4.2 of [2]. The assumption $\left(\mathrm{A}_{\infty}\right)$ in $\left[10\right.$, p. 226] also required $g^{\prime}(x) \rightarrow 0$ as $\|x\| \rightarrow \infty$.)

Claim 3.2. Under the assumption $\left(\mathrm{A}_{\infty}\right)$, suppose that $A_{0}$ has the finite dimensional kernel and negative definite subspace. Then the conditions of Corollary 1.6 are satisfied.

Proof. Since $0 \in \sigma\left(A_{0}\right)$ is isolated, there exists $\alpha>0$ such that $*\left(A u^{*}, u^{*}\right) \geq$ $2 \alpha\left\|u^{*}\right\|^{2}$ for all $u^{*} \in H^{*}, *=+,-$. By Lemma 3.1 we may write $A_{0}=$ 
$P(\infty)+Q(\infty)$, where $Q(\infty) \in L_{s}(H)$ is compact and $P(\infty) \in L_{s}(H)$ satisfies: $(P(\infty) u, u) \geq 2 \alpha\|u\|^{2}$ for all $u \in H$. We take $B(\infty):=A_{0}$. Choose $R>0$ so large that $\left\|g^{\prime \prime}(x)\right\| \leq \alpha$ as $\|x\| \geq R$. Since $B(x)=A_{0}+g^{\prime \prime}(x)=$ $P(\infty)+Q(\infty)+g^{\prime \prime}(x)$, we derive that

$$
([B(x)-Q(\infty)] u, u)_{H}=(P(\infty) u, u)_{H}+\left(g^{\prime \prime}(x) u, u\right)_{H} \geq \alpha\|u\|^{2}
$$

for all $u \in H$ and $x \in \bar{B}_{H}(\infty, R)$. Namely, the condition (c) of Corollary 1.6 is satisfied. Clearly, the condition (d) therein also holds since $B(x)-B(\infty)=$ $B(x)-A_{0}=g^{\prime \prime}(x) \rightarrow 0$ as $\|x\| \rightarrow \infty$.

That $A_{0}$ has a finite dimensional negative definite subspace corresponds to the finiteness of the Morse index at infinity, which is needed for computations of critical groups. The finiteness of $\operatorname{dim} \operatorname{Ker}\left(A_{0}\right)$ is naturally satisfied in the most actual applications. In this sense Claim 3.2 shows that Corollary 1.6 is a generalization of the splitting lemma at infinity on the page 431 of [2]. Our homeomorphism is not necessarily $C^{1}$-smooth, but we do not use the condition that $g$ and $g^{\prime}$ map bounded sets to bounded sets yet.

Consider the following weaker assumption than $\left(\mathrm{A}_{\infty}\right)$, which was given in Remark 2.3 of $[10$, p. 226]:

$\left(\mathrm{A}_{\infty}^{\prime}\right) f(x)=\left(A_{0} x, x\right)_{H} / 2+g(x)$ where $A_{0}: H \rightarrow H$ is a self-adjoint linear operator such that 0 is isolated in the spectrum of $A_{0}$. The map $g \in$ $C^{1}(H, \mathbb{R})$ is of class $C^{2}$ in a neighbourhood of infinity and satisfies: there exist $\alpha>0$ such that

$$
*\left(A_{0} u, u\right)_{H} \geq 2 \alpha\|u\|^{2} \quad \text { for all } u \in H^{*}, *=+,-
$$

and $\left\|g^{\prime \prime}\left(u^{0}+u^{ \pm}\right)\right\|<\alpha, \quad g^{\prime}\left(u^{0}+u^{ \pm}\right) \rightarrow 0 \quad$ as $\left\|u^{0}\right\| \rightarrow \infty$

where $H^{0}=\operatorname{Ker}\left(A_{0}\right)$ and $H^{+}$(resp. $H^{-}$) is the positive (resp. negative) definite subspace of $A_{0}$. Moreover, $g$ and $g^{\prime}$ map bounded sets to bounded sets.

Under the condition $\left(\mathrm{A}_{\infty}^{\prime}\right)$, Proposition 3.3 in [10] stated the following slightly different version of the splitting lemma of [2].

Theorem $3.3\left(\left[10\right.\right.$, Proposition 3.3]). For any $M>0$ there exist $R_{0}>0$, $\delta>0, a C^{1}$-diffeomorphism

$$
\psi: C_{R_{0}, M}=\left\{u=u^{0}+u^{ \pm} \mid\left\|u^{0}\right\|>R_{0},\left\|u^{ \pm}\right\|<M\right\} \rightarrow C_{R_{0}, 2 M}
$$

and a $C^{1}$-map $w: B_{H^{0}}\left(\infty, R_{0}\right) \rightarrow W^{\delta}=\left\{u^{ \pm} \in H^{ \pm} \mid\left\|u^{ \pm}\right\| \leq \delta\right\}$ such that

$$
f(\psi(u))=\frac{1}{2}\left(A_{0} w, w\right)_{H}+h\left(u^{0}\right) \quad \text { for all } u \in C_{R_{0}, M},
$$

where $h\left(u^{0}\right)=f\left(u^{0}+w\left(u^{0}\right)\right), \delta$ can be chosen as small as we please if we choose $R_{0}$ large, and $w=w\left(u^{0}\right)$ is the unique solution of $P^{ \pm} f^{\prime}\left(u^{0}+w\right)=0$. Furthermore, $\left(h^{\prime}\left(u^{0}\right), \xi\right)=\left(g^{\prime}\left(u^{0}+w\left(u^{0}\right)\right), \xi\right)$ for any $\xi \in H^{0}$. 
Note: It was stated in [10, p. 235] that one may refer to Lemma 4.3 and its proof in [2] for the first part of this theorem. Carefully checking the proof of its generalization in [6, Theorem 2.1] we believe that the diffeomorphism $\psi$ in this theorem and Theorem 3.5 below should actually be from $C_{R_{0}, M}$ onto an open subset $V$ of $C_{R_{0}, 2 M}$ (possibly satisfying $V \supseteq C_{R_{0}, r}$ for some $r>0$ ). In fact, the equation (2.19) in [6] is solved on ball $B_{E_{1}}(0,2 M)$ for each fixed $y \in Y$ with $\|y\|>R$. The condition that $\left\|\chi_{x, y}^{t}\right\|_{E} \leq\|x\|_{E} / 2$ implies that for each $x \in B_{E_{1}}(0, M)$ the initial value problem

$$
\frac{d}{d t} \eta(t)=\chi_{\eta(t), y}^{t}, \quad \eta(t)=x
$$

has a unique $C^{1}$-solution $\eta:[0,1] \rightarrow \eta(t, x, y) \in B_{E_{1}}(0,2 M)$, which depends $C^{1}$-smoothly on the parameter $(t, y)$ and initial value $x$. So $B_{E_{1}}(0, M) \ni x \mapsto$ $\eta(1, x, y) \in B_{E_{1}}(0,2 M)$ is a $C^{1}$-diffeomorphism from $B_{E_{1}}(0, M)$ onto some open neighbourhood of 0 in $B_{E_{1}}(0,2 M)$. The desired $\psi$ given by $\psi(x, y)=\eta(1, x, y)+$ $w(y)+y$, is a $C^{1}$-diffeomorphism from $C_{R_{0}, M}$ onto an open subset $V$ of $C_{R_{0}, 2 M}$ containing $\{y \in Y \mid\|y\|>R\}$. Since $\|w(y)\| \rightarrow 0$ as $\|y\| \rightarrow \infty$ it is possible to prove that for sufficiently large $R>0$ the image of $\psi$ contains some $C_{R_{0}, r}$ for small $r>0$.

ClaIm 3.4. Under the assumption $\left(\mathrm{A}_{\infty}^{\prime}\right)$, suppose that $A_{0}$ has the finite dimensional kernel and negative definite subspace. Then the conditions of Corollary 1.7 are satisfied.

Proof. Following the notations in the proof of Claim 3.2, since $\| g^{\prime \prime}\left(u^{0}+\right.$ $\left.u^{ \pm}\right) \|<\alpha$ for all $u^{0}+u^{ \pm}$, as in the proof of Claim 3.2 we may prove that the condition (c) is satisfied. It remains to prove that the condition (d) holds in the present case. Now $B(\infty)=A_{0}$ and $H_{\infty}^{*}=H^{*}, *=0,-,+$. Since $*(B(\infty) u, u)_{H} \geq$ $2 \alpha\|u\|^{2}$ for all $u \in H_{\infty}^{*}, *=+,-$, the restrictions $\left.B(\infty)\right|_{H_{\infty}^{*}}: H_{\infty}^{*} \rightarrow H_{\infty}^{*}$ are invertible and $\left\|\left(\left.B(\infty)\right|_{H_{\infty}^{*}}\right)^{-1}\right\| \leq 1 /(2 \alpha)$. Write $H_{\infty}^{ \pm}=H_{\infty}^{+} \oplus H_{\infty}^{-}$as before. Then $\left.B(\infty)\right|_{H_{\infty}^{ \pm}}: H_{\infty}^{ \pm} \rightarrow H_{\infty}^{ \pm}$is invertible and

$$
\left(\left.B(\infty)\right|_{H_{\infty}^{ \pm}}\right)^{-1}\left(u^{+}+u^{-}\right)=\left(\left.B(\infty)\right|_{H_{\infty}^{+}}\right)^{-1} u^{+}+\left(\left.B(\infty)\right|_{H_{\infty}^{-}}\right)^{-1} u^{-}
$$

for any $u^{+}+u^{-} \in H_{\infty}^{+}+H_{\infty}^{-}$. This leads to

$$
\begin{aligned}
\left\|\left(\left.B(\infty)\right|_{H_{\infty}^{ \pm}}\right)^{-1}\left(u^{+}+u^{-}\right)\right\|^{2} & =\left\|\left(\left.B(\infty)\right|_{H_{\infty}^{+}}\right)^{-1} u^{+}\right\|^{2}+\left\|\left(\left.B(\infty)\right|_{H_{\infty}^{-}}\right)^{-1} u^{-}\right\|^{2} \\
& \leq\left(\frac{1}{2 \alpha}\right)^{2}\left(\left\|u^{+}\right\|^{2}+\left\|u^{-}\right\|^{2}\right)
\end{aligned}
$$

and hence $C_{1}^{\infty}=\left\|\left(\left.B(\infty)\right|_{H_{\infty}^{ \pm}}\right)^{-1}\right\|_{L\left(H_{\infty}^{ \pm}\right)} \leq 1 /(2 \alpha)$. Since $B(x)-B(\infty)=g^{\prime \prime}(x)$,

$$
\left\|\left.B(z+y)\right|_{H_{\infty}^{ \pm}}-\left.B(\infty)\right|_{H_{\infty}^{ \pm}}\right\|_{L\left(H_{\infty}^{ \pm}\right)}=\left\|\left.g^{\prime \prime}(z+y)\right|_{H_{\infty}^{ \pm}}\right\|_{L\left(H_{\infty}^{ \pm}\right)}<\alpha \leq \frac{1}{2 C_{1}^{\infty}}
$$


for all $y \in H_{\infty}^{ \pm}$and $z \in H_{\infty}^{0}$. Hence the condition (d) holds with $\rho_{A}=\infty$. But $M(A)=0$ because $g^{\prime}\left(u^{0}+u^{ \pm}\right) \rightarrow 0$ as $\left\|u^{0}\right\| \rightarrow \infty$ (we here only need $g^{\prime}\left(u^{0}\right) \rightarrow 0$ as $\left.\left\|u^{0}\right\| \rightarrow \infty\right)$.

We can also take $\rho_{A}$ to be any given $\delta>0$ so that the $C^{1}$-map $w$ in Theorem 3.3 is assured to take values in $W^{\delta}=\left\{u^{ \pm} \in H^{ \pm} \mid\left\|u^{ \pm}\right\| \leq \delta\right\}$. Without the condition that $g^{\prime}\left(u^{0}\right) \rightarrow 0$ as $\left\|u^{0}\right\| \rightarrow \infty$, we may also derive Theorem 3.3 except claims that $\psi$ is $C^{1}$ and $w$ takes values in $W^{\delta}$.

Hence Claim 3.2 (and Note below Theorem 3.3) shows that Corollary 1.7 is a generalization of Theorem 3.3. We only need that

$$
\sup \left\|\left.g^{\prime \prime}(z+y)\right|_{H_{\infty}^{ \pm}}\right\|_{L\left(H_{\infty}^{ \pm}\right)} \leq \frac{1}{\kappa C_{1}^{\infty}} \text { for some } 1<\kappa \leq 2 .
$$

This is better than the condition that $\sup \left\|\left.g^{\prime \prime}(z+y)\right|_{H_{\infty}^{ \pm}}\right\|_{L\left(H_{\infty}^{ \pm}\right)} \leq \alpha \leq 1 /\left(2 C_{1}^{\infty}\right)$. Moreover, we do not use the condition that $g$ and $g^{\prime}$ map bounded sets to bounded sets.

3.2. Relations to the generalization version in [5]. For convenience of comparison with ours we briefly review it in our notations. Let $L: H \rightarrow H$ be a bounded self-adjoint linear operator. Let $H_{\infty}^{0}=\operatorname{Ker}(L)$ and $H_{\infty}^{ \pm}=\left(H_{\infty}^{0}\right)^{\perp}$. It was assumed in [5] that $L$ satisfies the condition

(L) The operator $\left.L\right|_{H_{\infty}^{ \pm}}: H_{\infty}^{ \pm} \rightarrow H_{\infty}^{ \pm}$is invertible and its inverse operator $\left(\left.L\right|_{H_{\infty}^{ \pm}}\right)^{-1}: H_{\infty}^{ \pm} \rightarrow H_{\infty}^{ \pm}$is bounded.

By Proposition 4.5 of $[7]$ this condition is equivalent to our $\left(\mathrm{C} 1_{\infty}\right)$, that is, 0 is at most an isolated point of the spectrum $\sigma(L)$. (See Proposition B.3 in [13], [14].)

Denote by $P_{\infty}^{0}$ the orthogonal projection onto $H_{\infty}^{0}$. (Then $I-P_{\infty}^{0}$ is such a projection onto $H_{\infty}^{ \pm}$.) For a $C^{2}$ functional $\mathcal{F}: H=H_{\infty}^{0} \oplus H_{\infty}^{ \pm} \rightarrow \mathbb{R}$, let $D^{2} \mathcal{F}(x)$ be the Hessian operator of it at a critical point $x$. For $z+u \in H$, where $z \in H_{\infty}^{0}$ and $u \in H_{\infty}^{ \pm}$, let $\nabla_{2} \mathcal{F}(z, u) \in H_{\infty}^{ \pm}$be defined by $\left(\nabla_{2} \mathcal{F}(z, u), v\right)_{H}=d_{u} \mathcal{F}(z, u)(v)$. Then

$$
\nabla_{2} \mathcal{F}(z, u)=\left(I-P_{\infty}^{0}\right) \nabla \mathcal{F}(z+u) .
$$

There exists a unique operator $\mathcal{J}(z, u) \in L_{s}\left(H_{\infty}^{ \pm}\right)$such that

$$
d_{u}^{2} \mathcal{F}(z, u)\left(v_{1}, v_{2}\right)=\left(\mathcal{J}(z, u) v_{1}, v_{2}\right)_{H} \quad \text { for all } v_{1}, v_{2} \in H_{\infty}^{ \pm} .
$$

It is easily seen that

$$
\mathcal{J}(z, u)=\left.\left(I-P_{\infty}^{0}\right) D(\nabla \mathcal{F})(z+u)\right|_{H_{\infty}^{ \pm}}
$$

because

$$
\begin{aligned}
d_{u}^{2} \mathcal{F}(z, u)\left(v_{1}, v_{2}\right) & =\left.\frac{\partial^{2}}{\partial s_{1} \partial s_{2}} \mathcal{F}\left(z, u+s_{1} v_{1}+s_{2} v_{2}\right)\right|_{s_{1}=0, s_{2}=0} \\
& =\left.\frac{d}{d s_{2}}\left(\nabla_{2} \mathcal{F}\left(z, u+s_{2} v_{2}\right), v_{1}\right)_{H}\right|_{s_{2}=0}
\end{aligned}
$$




$$
\begin{aligned}
& =\left.\frac{d}{d s_{2}}\left(\left(I-P_{\infty}^{0}\right) \nabla \mathcal{F}\left(z+u+s_{2} v_{2}\right), v_{1}\right)_{H}\right|_{s_{2}=0} \\
& =\left(\left(I-P_{\infty}^{0}\right) D(\nabla \mathcal{F})(z+u)\left(v_{2}\right), v_{1}\right)_{H} .
\end{aligned}
$$

Theorem 3.5 ([5, Theorem 2.1]). For the above functional $\mathcal{F}$ and operator $L$, suppose that there exists some $M>0$ such that as $\|z\| \rightarrow \infty$ one has

$\left(\mathrm{L}_{1}\right)\left\|\left(I-P_{\infty}^{0}\right) \nabla \mathcal{F}(z+u)-L u\right\| \rightarrow 0$ uniformly for $\|u\| \leq M$,

$\left(\mathrm{L}_{2}\right)\left\|\left.\left(I-P_{\infty}^{0}\right) D(\nabla \mathcal{F})(z+u)\right|_{H_{\infty}^{ \pm}}-\left.L\right|_{H_{\infty}^{ \pm}}\right\|_{L\left(H_{\infty}^{ \pm}\right)} \rightarrow 0$ uniformly for $\|u\| \leq M$.

Then there exist $R>0$, a $C^{1}$-homeomorphism

$\psi: C_{R, M}=\left\{z+u \mid z \in H_{\infty}^{0}, u \in H_{\infty}^{ \pm},\|z\| \geq R,\|u\| \leq M\right\} \rightarrow C_{R, 2 M}$ and a $C^{1}$-map $h^{\infty}: B_{H_{\infty}^{0}}(\infty, R) \rightarrow B_{H_{\infty}^{ \pm}}(\theta, M)$ such that

(a) $\mathcal{F}(\psi(z+u))=\frac{1}{2}(L u, u)_{H}+\mathcal{F}\left(z+h^{\infty}(z)\right)$ for all $z+u \in C_{R, M}$,

(b) $\left(I-P_{\infty}^{0}\right) \mathcal{F}\left(z+h^{\infty}(z)\right)=0$ for all $z \in B_{H_{\infty}^{0}}(\infty, R)$,

(c) $\left\|h^{\infty}(z)\right\| \rightarrow 0$ as $\|z\| \rightarrow \infty$.

The following condition is slightly stronger than $\left(\mathrm{L}_{2}\right)$.

$\left(\mathrm{L}_{2}^{\prime}\right)\left\|\left(I-P_{\infty}^{0}\right) D(\nabla \mathcal{F})(z+u)-L\right\|_{L\left(H, H_{\infty}^{ \pm}\right)} \rightarrow 0$ uniformly for $\|u\| \leq M$.

Take $X=H, A(z+u)=\nabla \mathcal{F}(z+u)$ and $B(\infty)=L$. By $\left(\mathrm{L}_{1}\right)$ we get

$$
M(A)=\lim _{R \rightarrow \infty} \sup \left\{\left\|\left(I-P_{\infty}^{0}\right) A(z)\right\|: z \in H^{0},\|z\| \geq R\right\}=0 .
$$

LEMMA 3.6.

(a) $\left(\mathrm{L}_{2}\right)$ implies that $\left(\mathrm{SE}_{\infty}^{\prime}\right)$ holds for $\rho_{A}=M>0=C_{1}^{\infty} M(A)$.

(b) $\left(\mathrm{L}_{1}\right)$ and $\left(\mathrm{L}_{2}^{\prime}\right)$ imply that $\left(\mathrm{SE}_{\infty}\right)$ holds for $\rho_{A}=M>0=C_{1}^{\infty} M(A)$.

Proof. (a) For any $z \in H_{\infty}^{0}$ and $u_{i} \in H_{\infty}^{-}$with $\left\|u_{i}\right\| \leq M, i=1,2$, using the mean value theorem in inequality form we derive

$$
\begin{aligned}
\|(I & \left.-P_{\infty}^{0}\right) A\left(z+u_{1}\right)-L u_{1}-\left(I-P_{\infty}^{0}\right) A\left(z+u_{2}\right)+L u_{2} \| \\
& \leq \sup _{t \in[0,1]}\left\|\left(I-P_{\infty}^{0}\right) D A\left(z+t u_{1}+(1-t) u_{2}\right)\left(u_{1}-u_{2}\right)-L\left(u_{1}-u_{2}\right)\right\| \\
& \leq \sup _{t \in[0,1]}\left\|\left.\left(I-P_{\infty}^{0}\right) D A\left(z+t u_{1}+(1-t) u_{2}\right)\right|_{H_{\infty}^{ \pm}}-\left.L\right|_{H_{\infty}^{ \pm}}\right\| \cdot\left\|u_{1}-u_{2}\right\| .
\end{aligned}
$$

From this it is easily seen that $\left(\mathrm{L}_{2}\right)$ leads to $\left(\mathrm{SE}_{\infty}^{\prime}\right)$ with $\rho_{A}=M$.

(b) For any given $\varepsilon>0$, by $\left(\mathrm{L}_{1}\right)$ and $\left(\mathrm{L}_{2}^{\prime}\right)$ there exists $R>3$ such that

$$
\begin{aligned}
\left\|\left(I-P_{\infty}^{0}\right) A(z+u)-L u\right\| & <M \varepsilon, \\
\left\|\left(I-P_{\infty}^{0}\right) D A(z+u)-L\right\|_{L\left(H, H_{\infty}^{ \pm}\right)} & <\varepsilon
\end{aligned}
$$

for any $u \in B_{H_{\infty}^{ \pm}}(\theta, M)$ and $z \in B_{H^{0}}(\infty, R)$. Hence for any $u_{i} \in B_{H_{\infty}^{ \pm}}(\theta, M)$ and $z_{i} \in B_{H^{0}}(\infty, R+4 M), i=1,2$, if $\left\|z_{1}-z_{2}\right\| \geq 3 M$ then from (3.3) we derive

$$
\begin{aligned}
\|\left(I-P_{\infty}^{0}\right) A\left(z_{1}+u_{1}\right)-L u_{1}-\left(I-P_{\infty}^{0}\right) A\left(z_{2}+u_{2}\right) & +L u_{2} \| \\
& \leq 2 M \varepsilon \leq 2 \varepsilon\left\|z_{1}+u_{1}-z_{2}-u_{2}\right\|
\end{aligned}
$$


because $\mid z_{1}+u_{1}-z_{2}-u_{2}\|\geq\| z_{1}-z_{2}\|-\| u_{1}-u_{2}\|\geq\| z_{1}-z_{2} \|-2 M \geq M$; and if $\left\|z_{1}-z_{2}\right\|<3 M$ using the mean value theorem we get a number $t \in(0,1)$ such that

$$
\begin{aligned}
\| & \left(I-P_{\infty}^{0}\right) A\left(z_{1}+x_{1}\right)-L x_{1}-\left(I-P_{\infty}^{0}\right) A\left(z_{2}+x_{2}\right)+L x_{2} \| \\
\leq & \|\left(I-P_{\infty}^{0}\right) D A\left(t z_{1}+(1-t) z_{2}+t x_{1}+(1-t) x_{2}\right)\left(z_{1}+x_{1}-z_{2}-x_{2}\right) \\
& \quad-L\left(z_{1}+x_{1}-z_{2}-x_{2}\right) \| \\
\leq & \left\|\left(I-P_{\infty}^{0}\right) D A\left(t z_{1}+(1-t) z_{2}+t x_{1}+(1-t) x_{2}\right)-L\right\| \cdot\left\|z_{1}+x_{1}-z_{2}-x_{2}\right\| \\
\leq & \varepsilon\left\|z_{1}+x_{1}-z_{2}-x_{2}\right\|
\end{aligned}
$$

by (3.4), because $\left\|t z_{1}+(1-t) z_{2}\right\| \geq\left\|z_{2}\right\|-\left\|z_{1}-z_{2}\right\|>R+4 M-3 M \geq R+M$, (b) follows.

Take $B(z+u)=\mathcal{F}^{\prime \prime}(z+u)=D A(z+u)$. We have

Lemma 3.7. $\left(\mathrm{L}_{1}\right)$ and $\left(\mathrm{L}_{2}^{\prime}\right)$ imply that $\left(\mathrm{D}_{\infty}^{\prime \prime}\right)$ in Remark 1.4 holds for $X=H$. Moreover, if $M=\infty$ in $\left(\mathrm{L}_{1}\right)$ and $\left(\mathrm{L}_{2}^{\prime}\right)$ then $\left(\mathrm{D}_{\infty}^{\prime}\right)$ in Remark 1.2 holds for $X=H$.

Proof. Let $B(x)=D(\nabla \mathcal{F})(x)$ and $B(\infty)=L$. Since 0 is at most an isolated point in $\sigma(L)$, we have a positive number $a_{\infty}>0$ such that

$$
\begin{array}{ll}
(L u, u)_{H} \geq 2 a_{\infty}\|u\|^{2} & \text { for all } u \in H_{\infty}^{+}, \\
(L u, u)_{H} \leq-2 a_{\infty}\|u\|^{2} & \text { for all } u \in H_{\infty}^{-} .
\end{array}
$$

By $\left(\mathrm{L}_{2}^{\prime}\right)$ we have a number $R_{0}>0$ such that

$$
\left\|\left(I-P_{\infty}^{0}\right) B(z+u)-L\right\|_{L\left(H, H_{\infty}^{ \pm}\right)}<a_{\infty} \quad \text { for all }(z, u) \in W_{\infty},
$$

where $W_{\infty}:=B_{H_{\infty}^{0}}\left(\infty, R_{0}\right) \times B_{H_{\infty}^{ \pm}}(\theta, M)$. Set

$$
\omega_{\infty}: W_{\infty} \rightarrow[0, \infty), x \mapsto\left\|\left(I-P_{\infty}^{0}\right) B(x)-L\right\|_{L\left(H, H_{\infty}^{ \pm}\right)} .
$$

Then $\left(\mathrm{L}_{2}^{\prime}\right)$ implies that $\omega_{\infty}(x) \rightarrow 0$ as $x \in W_{\infty}$ and $\|x\| \rightarrow \infty$.

For $x \in W_{\infty}$ and $v \in H_{\infty}^{+}$, we have

$$
\begin{aligned}
(B(x) v, v)_{H} & =\left(B(x) v,\left(I-P_{\infty}^{0}\right) v\right)_{H}=\left(\left(I-P_{\infty}^{0}\right) B(x) v, v\right)_{H} \\
& =(L v, v)_{H}+\left(\left(I-P_{\infty}^{0}\right) B(x) v-L v, v\right)_{H} \\
& \geq 2 a_{\infty}\|v\|^{2}-\left\|\left(I-P_{\infty}^{0}\right) B(x)-L\right\| \cdot\|v\|^{2} \geq a_{\infty}\|v\|^{2}
\end{aligned}
$$

because (3.5). Similarly, for all $x \in W_{\infty}$ and $v \in H_{\infty}^{-}$we have

$$
(B(x) v, v)_{H}=\left(B(x) v,\left(I-P_{\infty}^{0}\right) v\right)_{H}=\left(\left(I-P_{\infty}^{0}\right) B(x) v, v\right)_{H} \leq-a_{\infty}\|v\|^{2} .
$$

Finally, for all $x \in W_{\infty}, u \in H$ and $v \in H_{\infty}^{ \pm}$, we get

$$
\begin{aligned}
\left|(B(x) u, v)_{H}-(B(\infty) u, v)_{H}\right| & =\left|\left(B(x) u-B(\infty) u,\left(I-P_{\infty}^{0}\right) v\right)_{H}\right| \\
& =\left|\left(\left(I-P_{\infty}^{0}\right) B(x) u-\left(I-P_{\infty}^{0}\right) L u, v\right)_{H}\right| \\
& =\left|\left(\left(I-P_{\infty}^{0}\right)[B(x)-L] u, v\right)_{H}\right| \leq \omega_{\infty}(x)\|u\| \cdot\|v\|
\end{aligned}
$$


since $\left(I-P_{\infty}^{0}\right) L u=L\left(I-P_{\infty}^{0}\right) u=L u$. The second claim is easily seen from the proof above.

By Lemmas 3.6 and 3.7, under the assumptions $\left(\mathrm{L}_{1}\right)$ and $\left(\mathrm{L}_{2}^{\prime}\right)$, if $L$ has the finite dimensional kernel and negative definite subspace, then Theorem 3.5 follows from Theorem 1.3 with $X=H$ by Remark 1.4 unless our homeomorphism is not necessarily $C^{1}$-smooth. Furthermore, if $M=\infty$ in $\left(\mathrm{L}_{1}\right)$ and $\left(\mathrm{L}_{2}^{\prime}\right)$ a stronger result follows from Remark 1.2, that is, there exist a positive number $R$, a (unique) continuous map $h^{\infty}: B_{H_{\infty}^{0}}(\infty, R) \rightarrow X_{\infty}^{ \pm}$satisfying (1.6), and a homeomorphism $\phi: B_{H_{\infty}^{0}}(\infty, R) \oplus H_{\infty}^{ \pm} \rightarrow \bar{B}_{H_{\infty}^{0}}(\infty, R) \oplus H_{\infty}^{ \pm}$of form (1.7) such that (1.8) and (a)-(e) in Theorem 1.1 hold.

Note: $\left(\mathrm{L}_{1}\right)+\left(\mathrm{L}_{2}^{\prime}\right)=\left(\mathrm{L}_{1}\right)+\left(\mathrm{L}_{2}\right)+$ the following $(3.6)$, where

$$
\left\{\begin{array}{l}
\left\|\left.\left(I-P_{\infty}^{0}\right) D(\nabla \mathcal{F})(z+u)\right|_{H_{\infty}^{0}}\right\|_{L\left(H_{\infty}^{0}, H_{\infty}^{ \pm}\right)} \rightarrow 0 \\
\text { uniformly for }\|u\| \leq M \text { as } z \in H_{\infty}^{0} \text { and }\|z\| \rightarrow \infty
\end{array}\right.
$$

\section{A simple application}

To save the length of this paper we are only satisfied with a simple application of generalizing Theorem 5.2 in [2]. Some of the results in [10], [12], [5] may be generalized with the similar ideas. They shall be given in other places.

Let $\Omega \subset \mathbb{R}^{n}$ be a bounded open domain with $C^{2}$-boundary $\partial \Omega$, and let $p: \Omega \times \mathbb{R} \rightarrow \mathbb{R}$ be a Carthéodory function satisfying $p(x, 0)=0$ for all $x \in \Omega$ and the following condition:

(p) $a_{0}=\lim _{t \rightarrow 0} p(x, t) / t$ uniformly in $x \in \Omega$, and $a=\lim _{|t| \rightarrow \infty} p(x, t) / t$ uniformly in $x \in \Omega$.

Consider the BVP

$$
-\triangle u=p(\cdot, u) \text { in } \Omega \text { and }\left.\quad u\right|_{\partial \Omega}=0 .
$$

It is called non-resonant at infinity if $a$ is not an eigenvalue of $-\triangle$ with 0 boundary conditions. Let $q(x, t)=p(x, t)-a t, q_{0}(x, t)=p(x, t)-a_{0} t$, and

$$
Q(x, t)=\int_{0}^{t} q(x, \tau) d \tau, \quad Q_{0}(x, t)=\int_{0}^{t} q_{0}(x, \tau) d \tau .
$$

Here are the hypotheses on $q$ given in [2].

( $\left.\mathrm{q}_{1}\right)$ There exist constants $c_{1}>0$ and $r \in(0,1)$ such that

$$
|q(x, t)| \leq c_{1}\left(1+|t|^{r}\right) \quad \text { for all }(x, t) \in \Omega \times \mathbb{R} \text {; }
$$

$\left(\mathrm{q}_{2}\right)$ There exist constants $c_{2}>0$ and $\alpha>1$ such that either

$$
Q(x, t)-\frac{1}{2} q(x, t) t \geq c_{2}\left(|t|^{\alpha}-1\right) \quad \text { for all }(x, t) \in \Omega \times \mathbb{R},
$$


or

$$
\frac{1}{2} q(x, t) t-Q(x, t) \geq c_{2}\left(|t|^{\alpha}-1\right) \quad \text { for all }(x, t) \in \Omega \times \mathbb{R} ;
$$

$\left(\mathrm{q}_{3}\right) q \in C^{1}(\bar{\Omega} \times \mathbb{R})$ and $\frac{\partial}{\partial t} q(x, t) \rightarrow 0$ as $|t| \rightarrow \infty$ uniformly in $x \in \Omega$;

$\left(\mathrm{q}_{4}^{ \pm}\right) \pm Q_{0}(x, t)>0$ for $|t|>0$ small, $x \in \Omega$;

TheOREM 4.1 ([2, Theorem 5.2]). Let the assumptions $(\mathrm{p})$ and $\left(\mathrm{q}_{1}\right)-\left(\mathrm{q}_{3}\right)$ be satisfied.

(a) If $a_{0}$ is not an eigenvalue of $-\triangle$ then (4.1) has at least one nontrivial solution provided $a_{0}<\lambda_{m}<a$ or $a<\lambda_{m}<a_{0}$ for some $m \in \mathbb{N}$.

(b) If $a_{0}=\lambda_{m}$ is an eigenvalue but $\left(\mathrm{q}_{4}^{+}\right)$holds in addition, then (4.1) has at least one nontrivial solution provided $a<a_{0}$ or $a_{0}<\lambda_{k}<a$ for some $k>m$.

(c) If $a_{0}=\lambda_{m}$ is an eigenvalue but ( $\left.\mathrm{q}_{4}^{-}\right)$holds in addition, then (4.1) has at least one nontrivial solution provided $a_{0}<a$ or $a<\lambda_{k}<a_{0}$ for some $k<m$.

We wish to prove this theorem provided that the conditions $(\mathrm{p})$ and $\left(\mathrm{q}_{1}\right)-\left(\mathrm{q}_{3}\right)$ are replaced by the following four respective weaker ones

$\left(\mathrm{p}^{*}\right) a_{0}=\lim _{t \rightarrow 0} p(x, t) / t$ for almost every $x \in \Omega$ and $a=\lim _{|t| \rightarrow \infty} p(x, t) / t$ for almost every $x \in \Omega$.

$\left(\mathrm{q}_{1}^{*}\right)$ There exist constants $c_{1}>0, r \in(0,1)$ and a function $E \in L^{1}(\Omega)$ such that

$$
|q(x, t)| \leq E(x)+c_{1}|t|^{r} \quad \text { for all }(x, t) \in \Omega \times \mathbb{R} ;
$$

$\left(\mathrm{q}_{2}^{*}\right)$ There exist constants $c_{2}>0, \alpha>1$ and $G \in L^{1}(\Omega)$ such that either

$$
\begin{aligned}
& Q(x, t)-\frac{1}{2} q(x, t) t \geq c_{2}|t|^{\alpha}-G(x) \quad \text { for all }(x, t) \in \Omega \times \mathbb{R} \text {, } \\
& \text { or } \quad \frac{1}{2} q(x, t) t-Q(x, t) \geq c_{2}|t|^{\alpha}-G(x) \quad \text { for all }(x, t) \in \Omega \times \mathbb{R} ;
\end{aligned}
$$

$\left(\mathrm{q}_{3}^{*}\right)$ For almost every $x \in \Omega$ the function $\mathbb{R} \ni t \mapsto q(x, t)$ is differentiable and $\Omega \times \mathbb{R} \ni(x, t) \mapsto q_{t}(x, t)$ is a Carthéodory function. Moreover, there exist $s \in(2 n /(n+2), 2 n /(n-2))$ in case $n>2$ and $s \in(2 n /(n+2), \infty)$ in case $n=2$, and $\ell \in L^{s}(\Omega)$, a bounded measurable $h: \mathbb{R} \rightarrow \mathbb{R}$ such that $h(t) \rightarrow \hbar \in \mathbb{R}$ as $|t| \rightarrow \infty$ and $\left|q_{t}(x, t)\right| \leq \ell(x) h(t)$ for almost every $x \in \Omega$ and for almost all $t \in \mathbb{R}$. (Clearly, $h \geq 0$ and $\hbar \geq 0$.)

Since $q(x, 0)=0$ and $a t+q(x, t)=p(x, t)=a_{0} t+q_{0}(x, t)$ by the definition, $\left(\mathrm{p}^{*}\right)$ and $\left(\mathrm{q}_{3}^{*}\right)$ imply that for almost every $x \in \Omega$, the derivative $q_{t}(x, 0)$ exists and $a_{0}=a+q_{t}(x, 0)$. 
For $q$ in $\left(\mathrm{q}_{3}\right)$ let $h(t):=\max _{x \bar{\Omega}}\left|\frac{\partial}{\partial t} q(x, t)\right|$ for each $t \in \mathbb{R}$. It is easily proved that $h \in L^{\infty}(\mathbb{R})$ and $h(t) \rightarrow 0$ as $|t| \rightarrow \infty$. This shows that $q$ satisfies $\left(\mathrm{q}_{3}^{*}\right)$. On the other hand, for $q$ in $\left(\mathrm{q}_{3}^{*}\right)$ we cannot deduce that $\frac{\partial}{\partial t} q(x, t) \rightarrow 0$ as $|t| \rightarrow \infty$ uniformly in $x \in \Omega$ in case $\hbar=0$ (even if we also assume $q \in C^{1}(\bar{\Omega} \times \mathbb{R}$ ).) Hence the condition $\left(\mathrm{q}_{3}^{*}\right)$ is much weaker than $\left(\mathrm{q}_{3}\right)$.

Recall that the Laplacian $-\triangle$ is a self-adjoint operator defined on $L^{2}(\Omega)$, with domain $D(-\triangle)=H^{2}(\Omega) \cap H_{0}^{1}(\Omega)$. By the standard theory of linear elliptic BDV due to Agmon-Douglis-Nirenberg, for any $1<s<\infty$ the Laplacian $-\triangle$ can be extended into a continuous Fredholm operator from $W^{2, s}(\Omega) \cap W_{0}^{1, s}(\Omega)$ to $L^{s}(\Omega)$. Moreover, $-\triangle$ is invertible and $K=(-\triangle)^{-1}$ is a continuous linear operator from $L^{s}(\Omega)$ to $W^{2, s}(\Omega)$ (see Brezis [3], Theorem 9.32). Clearly, $K$ satisfies $(u, v)_{L^{2}}=$ $(K u, v)_{H_{0}^{1}}$ for any $v \in H_{0}^{1}(\Omega)$ and $u \in L^{2}(\Omega)$, where $(w, v)_{H_{0}^{1}}=\int_{\Omega} \nabla w \cdot \nabla v d x$. Note that $K$ is also a positive, self-adjoint and completely continuous operator from $L^{2}(\Omega)$ to $L^{2}(\Omega)$ (resp. from $H_{0}^{1}(\Omega)$ to $H_{0}^{1}(\Omega)$ ). The eigenvalues of the Laplacian $-\triangle$ on $\Omega$ with 0 boundary conditions form an increasing sequence: $0<\lambda_{1} \leq \lambda_{2} \leq \ldots$, and $\lambda_{n} \rightarrow \infty$. (Actually, $\lambda_{1}<\lambda_{2}$ ). $K: H_{0}^{1}(\Omega) \rightarrow H_{0}^{1}(\Omega)$ has a countable set of eigenvalues $\left\{\mu_{n}\right\}_{n=1}^{\infty}=\left\{1 / \lambda_{n}\right\}_{n=1}^{\infty}$ of finite multiplicity.

Let $c(\Omega)$ be the smallest positive constant such that

$$
\|u\|_{C^{0}} \leq c(\Omega)\|\nabla u\|_{2}=c(\Omega)\|u\|_{H} \quad \text { for all } u \in H_{0}^{1}(\Omega)
$$

(because $H_{0}^{1}(\Omega) \hookrightarrow C(\bar{\Omega})$ ). For the $s \in \mathbb{R}$ in $\left(\mathrm{q}_{3}^{*}\right)$ we have the sequence of operators

$$
H_{0}^{1}(\Omega) \hookrightarrow L^{s}(\Omega) \stackrel{K}{\longrightarrow} W^{2, s}(\Omega) \cap W_{0}^{1, s}(\Omega) \hookrightarrow H_{0}^{1}(\Omega),
$$

where the first and the last are two embedding operators and the second is a bounded linear operator. By $\left(\mathrm{q}_{3}^{*}\right)$, for any $u, v \in H_{0}^{1}(\Omega)$, the function

$$
\Omega \ni x \mapsto q_{t}(x, u(x)) v(x) \quad \text { belongs to } L^{s}(\Omega),
$$

and for almost every $x \in \Omega$ the functions $\mathbb{R} \ni t \mapsto q(x, t)$ and $\mathbb{R} \ni t \mapsto q_{t}(x, t)$ are continuous. The calculus fundamental theorem leads to

$$
\left|q\left(x, t_{2}\right)-q\left(x, t_{1}\right)\right|=\left|\int_{t_{1}}^{t_{2}} q_{\tau}(x, \tau) d \tau\right| \leq\|h\|_{L^{\infty} \ell} \ell(x)\left|t_{2}-t_{1}\right|
$$

for all $t_{1}, t_{2} \in \mathbb{R}$, and hence

$$
|Q(x, t)| \leq \frac{1}{2}\|h\|_{L^{\infty}} \ell(x) t^{2} \quad \text { for a.e. } x \in \Omega, \text { for all } t \in \mathbb{R}
$$

because $q(x, 0)=0$. Hereafter $\|h\|_{\infty}=\sup \{|h(t)|: t \in \mathbb{R}\}$.

Let $H=H_{0}^{1}(\Omega)$ for convenience. Consider the functional

$$
J(u)=\int_{\Omega}\left(\frac{1}{2}|\nabla u|^{2}-\frac{1}{2} a u^{2}-Q(x, u(x))\right) d x \quad \text { for all } u \in H,
$$


and the bounded linear self-adjoint operator

$$
B(\infty): H_{0}^{1}(\Omega) \rightarrow H_{0}^{1}(\Omega), \quad u \mapsto u-a K u .
$$

Then $J(u)=(B(\infty) u, u)_{H} / 2+g(u)$ for all $u \in H$, where the functional

$$
g: H \rightarrow \mathbb{R}, \quad u \mapsto-\int_{\Omega} Q(x, u(x)) d x .
$$

For $u \in H$ define $B(u): H \rightarrow H$ by

$$
B(u) v=v-a K v-K\left(\bar{q}_{t}(u) v\right) \text { for all } v \in H_{0}^{1}(\Omega),
$$

where $\bar{q}_{t}(u)(x)=q_{t}(x, u(x))$ for all $x \in \bar{\Omega}$. Then for any $v, w \in H$ it holds that

$$
\begin{aligned}
(B(u) v, w)_{H} & =(v, w)_{H}-a(K v, w)_{H}-\left(K\left(\bar{q}_{t}(u) v\right), w\right)_{H} \\
& =(v, w)_{H}-a \int_{\Omega} v(x) w(x) d x-\int_{\Omega} q_{t}(x, u(x)) v(x) w(x) d x .
\end{aligned}
$$

It follows from $\left(\mathrm{q}_{3}^{*}\right)$ that $B(u) \in L_{s}(H)$ because

$$
\left|\int_{\Omega} q_{t}(x, u(x)) v(x) w(x) d x\right| \leq\left(\int_{\Omega} \ell(x) d x\right) \cdot\|h\|_{L^{\infty}} \cdot\|v\|_{C^{0}} \cdot\|w\|_{C^{0}} .
$$

Proposition 4.2. Suppose that the condition $\left(\mathrm{p}^{*}\right)$ is satisfied. Then:

(a) Under the assumption $\left(\mathrm{q}_{1}^{*}\right)$ the functional $J$ is $C^{1}$, and

$$
g(u)=J(u)-\frac{1}{2}(B(\infty) u, u)_{H}=o\left(\|u\|_{H}^{2}\right) \quad \text { as }\|u\|_{H} \rightarrow \infty .
$$

Moreover, $\nabla J(u)=u-a K u+\nabla g(u)=u-a K u-K(\bar{q}(u))$, where $\bar{q}(u)(x)=q(x, u(x))$ for $u \in H$.

(b) Under the assumption $\left(\mathrm{q}_{3}^{*}\right)$, $J$ is $C^{2}$ and $D(\nabla J)(u)=B(u)$ for all $u \in H$. Moreover, if $a=\lambda_{m}$, for any $z \in H_{\infty}^{0}=\operatorname{Ker}(B(\infty))$ and $u \in H_{\infty}^{ \pm}:=$ $\left(H_{\infty}^{0}\right)^{\perp}$ it holds that

(4.11) $\left\|g^{\prime \prime}(z+u)\right\|_{L(H)} \leq c(\Omega)^{2}\|\ell\|_{s}\left[\|h(z+u)-\hbar\|_{s_{1} s /\left(s-s_{1}\right)}+\hbar|\Omega|^{\left(s-s_{1}\right) /\left(s_{1} s\right)}\right]$, where $|\Omega|:=\operatorname{mes}(\Omega), 2 n /(n+2)<s_{1}<s$ and $s_{1}<2 n /(n-2)$ in case $n>2, c(\Omega)$ is as in $(4.2)$.

(c) Under the assumptions $\left(\mathrm{q}_{1}^{*}\right)$ and $\left(\mathrm{q}_{3}^{*}\right)$,

$$
\|\nabla J(u)-B(\infty) u\|_{H}=o\left(\|u\|_{H}\right) \quad \text { as }\|u\|_{H} \rightarrow \infty .
$$

(d) Under the assumptions $\left(\mathrm{q}_{1}^{*}\right)$ and $\left(\mathrm{q}_{2}^{*}\right)$ the functional $J$ satisfies the $P a$ lais-Smale condition.

Its proof is almost standard. For completeness we shall give it at the end of this section.

For simplicity we set

$$
s_{1}=\frac{1}{2}\left(\frac{2 n}{n+2}+\min \left\{s, \frac{2 n}{n-2}\right\}\right) \quad \text { and } \quad \iota(s)=\frac{s-s_{1}}{s_{1} s} .
$$


Lemma 4.3 ([1, Lemma 3.2]). Let $V$ be a finite dimensional subspace of $C(\bar{\Omega})$ such that every $v \in V \backslash\{0\}$ is different from zero almost everywhere in $\Omega$. Let $h \in L^{\infty}(\mathbb{R})$ such that $h(t) \rightarrow 0$ as $|t| \rightarrow \infty$. Moreover, consider a compact subset $K$ of $L^{p}(\Omega)(p \geq 1)$. Then

$$
\lim _{|t| \rightarrow \infty} \int_{\Omega}|h(t v(x)+u(x))| d x=0
$$

uniformly as $u \in K$ and $v \in S$, where $S=\left\{v \in V \mid\|v\|_{C^{0}}=1\right\}$.

Since any two norms on a finite dimensional linear space are equivalent, and any bounded set in $H=H_{0}^{1}(\Omega)$ is compact $L^{1}(\Omega)$, using this lemma we easily prove

Claim 4.4. For given numbers $\rho>0$ and $\varepsilon>0$ there exists a $R_{0}>0$ such that

$$
\sup _{\tau \in[0,1]}\|h(z+u)-\hbar\|_{s_{1} s /\left(s-s_{1}\right)}+\hbar|\Omega|^{\iota(s)}<\varepsilon+\hbar|\Omega|^{\iota(s)}
$$

for any $u \in \bar{B}_{H_{\infty}^{ \pm}}(\theta, \rho)$ and $z \in H_{\infty}^{0}$ with $\|z\|_{H} \geq R_{0}$.

Clearly, the origin $\theta$ of $H$ is a critical point of $J$, and $J^{\prime \prime}(\theta): H \rightarrow H$ is given by $J^{\prime \prime}(\theta) v=v-a_{0} K v$ for $v \in H=H_{0}^{1}(\Omega)$. Denote by

$$
H_{\theta}^{0}, H_{\theta}^{+}, H_{\theta}^{-} \quad\left(\text { resp. } H_{\infty}^{0}, H_{\infty}^{+}, H_{\infty}^{-}\right)
$$

the kernel, positive and negative definite subspaces of $J^{\prime \prime}(\theta)$ (resp. $B(\infty)$ ). Then $H=H_{\theta}^{0} \oplus H_{\theta}^{+} \oplus H_{\theta}^{-}$and $H=H_{\infty}^{0} \oplus H_{\infty}^{+} \oplus H_{\infty}^{-}$. Both $H_{\theta}^{0} \oplus H_{\theta}^{+}$and $H_{\infty}^{0} \oplus H_{\infty}^{+}$ are finite dimensional. Let $\nu_{\theta}=\operatorname{dim} H_{\theta}^{0}, \mu_{\theta}=\operatorname{dim} H_{\theta}^{-}$and $\nu_{\infty}=\operatorname{dim} H_{\infty}^{0}$, $\mu_{\infty}=\operatorname{dim} H_{\infty}^{-}$. They are the nullity and Morse index of $J$ at $\theta$ (resp. $\infty$ ). For $m \in \mathbb{N}$ let

$$
m^{-}=\min \left\{j \in \mathbb{N} \mid \lambda_{j}=\lambda_{m}\right\} \quad \text { and } \quad m^{+}=\max \left\{j \in \mathbb{N} \mid \lambda_{j}=\lambda_{m}\right\} .
$$

Clearly, $m^{-}=m^{+}=1$ for $m=1$, and $m^{-}=2$ for $m=2$. Let $\left\{\varphi_{j}\right\}_{j=1}^{\infty}$ be a normal orthogonal basis of $H=H_{0}^{1}(\Omega)$ consisting of the eigenfunctions associated with the eigenvalues $\left\{\lambda_{j}\right\}_{j=1}^{\infty}$. (So $\lambda_{j} \int_{\Omega}\left|\varphi_{j}(x)\right|^{2} d x=\int_{\Omega}\left|\nabla \varphi_{j}(x)\right|^{2} d x=1$ for all $j \in \mathbb{N}$.) Note that

$$
J^{\prime \prime}(\theta) \varphi_{j}=\frac{\lambda_{j}-a_{0}}{\lambda_{j}} \varphi_{j}, \quad B(\infty) \varphi_{j}=\frac{\lambda_{j}-a}{\lambda_{j}} \varphi_{j} \quad \text { for all } j \in \mathbb{N} .
$$

Clearly, $H_{\theta}^{0} \neq\{\theta\}$ (resp. $H_{\infty}^{0} \neq\{\theta\}$ ) if and only if $a_{0} \in\left\{\lambda_{m}\right\}_{m=1}^{\infty}$ (resp. $\left.a \in\left\{\lambda_{m}\right\}_{m=1}^{\infty}\right)$. If $a_{0}=a=\lambda_{m}$ then $H_{\theta}^{0}=H_{\infty}^{0}=\operatorname{Span}\left(\left\{\varphi_{j} \mid m^{-} \leq j \leq m^{+}\right\}\right)$ and

$$
H_{\theta}^{-}=H_{\infty}^{-}=\operatorname{Span}\left(\left\{\varphi_{j} \mid j<m^{-}\right\}\right) \quad \text { and } \quad H_{\theta}^{+}=H_{\infty}^{+}=\operatorname{Span}\left(\left\{\varphi_{j} \mid j>m^{+}\right\}\right) .
$$

If $a_{0}=a>\lambda_{1}$ and $a_{0}=a \notin\left\{\lambda_{i}\right\}_{i=1}^{\infty}$, then there exists $m \in \mathbb{N}$ such that $a_{0}=a \in\left(\lambda_{m^{+}}, \lambda_{m^{+}+1}\right)$ because $\lambda_{n} \rightarrow \infty$. In the case

$$
H_{\theta}^{-}=H_{\infty}^{-}=\operatorname{Span}\left(\left\{\varphi_{j} \mid j \leq m^{+}\right\}\right) \quad \text { and } \quad H_{\theta}^{+}=H_{\infty}^{+}=\operatorname{Span}\left(\left\{\varphi_{j} \mid j>m^{+}\right\}\right) \text {. }
$$


Hence we obtain

$$
\begin{aligned}
& \left.\begin{array}{l}
\nu_{\theta}=\nu_{\infty}=m^{+}-m^{-}+1, \\
\mu_{\theta}=\mu_{\infty}=m^{-}-1
\end{array}\right\} \quad \text { if } a_{0}=a=\lambda_{m}, \\
& \nu_{\theta}=\nu_{\infty}=0 \quad \text { and } \quad \mu_{\theta}=\mu_{\infty}=0 \quad \text { if } a_{0}=a<\lambda_{1} \text {, } \\
& \nu_{\theta}=\nu_{\infty}=0 \quad \text { and } \quad \mu_{\theta}=\mu_{\infty}=m^{+} \quad \text { if } a_{0}=a \in\left(\lambda_{m^{+}}, \lambda_{m^{+}+1}\right) .
\end{aligned}
$$

By the splitting theorem for $C^{2}$ functionals on Hilbert spaces (cf. [4], [16]) we get

Proposition 4.5 .

(a) If $a_{0}<\lambda_{1}$, then $C_{k}(J, \theta ; \mathbb{K})=\delta_{0 k} \mathbb{K}$.

(b) If $a_{0} \in\left(\lambda_{m^{+}}, \lambda_{m^{+}+1}\right)$ for some $m \in \mathbb{N}$, then $C_{k}(J, \theta ; \mathbb{K})=\delta_{m^{+} k} \mathbb{K}$.

(c) If $a_{0}=\lambda_{m}$, then $C_{k}(J, \theta ; \mathbb{K})=0$ for all $k \notin\left[m^{-}-1, m^{+}\right]$.

Under the assumptions of Proposition 4.2(d) the functional $J$ satisfies the Palais-Smale condition and hence the deformation condition $(D)_{c}$ at every $c \in \mathbb{R}$. Then the critical group of $J$ at infinity, $C_{*}(J, \infty ; \mathbb{K})$, is well-defined. The following is a generalization of Theorem 3.9 in [2].

Proposition 4.6. Let the assumptions of Proposition 4.2 be satisfied.

(a) If $a<\lambda_{1}$, then $C_{k}(J, \infty ; \mathbb{K})=\delta_{0 k} \mathbb{K}$.

(b) If $a \in\left(\lambda_{m^{+}}, \lambda_{m^{+}+1}\right)$ for $m \in \mathbb{N}$, then $C_{k}(J, \infty ; \mathbb{K})=\delta_{m^{+}{ }_{k}} \mathbb{K}$.

(c) If $a=\lambda_{m}$, then $C_{k}(J, \infty ; \mathbb{K})=0$ for all $k \notin\left[m^{-}-1, m^{+}\right]$provided that

$$
\hbar|\Omega|^{\iota(s)}\|\ell\|_{s}< \begin{cases}\frac{1}{c(\Omega)^{2}} \frac{\lambda_{2}-\lambda_{1}}{\lambda_{2}}, & m=1, \\ \frac{1}{c(\Omega)^{2}} \min \left\{\frac{\lambda_{m}-\lambda_{m^{-}-1}}{\lambda_{m^{-}-1}}, \frac{\lambda_{m^{+}+1}-\lambda_{2}}{\lambda_{m^{+}+1}}\right\}, & m>1,\end{cases}
$$

and

$$
\|\ell\|_{1} \cdot\|h\|_{L^{\infty}}<\frac{1}{c(\Omega)^{2}} .
$$

Here $\iota(s)$ is given by (4.13).

Clearly, when $\hbar=0$ the condition (4.14) is naturally satisfied because the left side of the inequality is always positive. If $\hbar>0$ the choice of $s_{1}$ in (4.13) shows that the upper bound of $\hbar$ given by (4.14) is not the biggest one.

Before proving it we point out that using Propositions 2.3, 3.6 in [2] and Propositions 4.5, 4.6 and repeating the proof of [2, Proposition 5.2] may lead to the following generalization for Theorem 4.1 .

THEOREM 4.7. Suppose that the assumptions $\left(\mathrm{p}^{*}\right)$ and $\left(\mathrm{q}_{1}^{*}\right)-\left(\mathrm{q}_{3}^{*}\right)$ are satisfied.

(a) If $a_{0}$ is not an eigenvalue of $-\triangle$ then (4.1) has at least one nontrivial solution provided that for some $m \in \mathbb{N}$, (4.14)-(4.15) hold and either $a_{0}<\lambda_{m}<a$ or $a<\lambda_{m}<a_{0}$. 
(b) If $a_{0}=\lambda_{m}$ is an eigenvalue but (4.14)-(4.15) and $\left(\mathrm{q}_{4}^{+}\right)$hold in addition, then (4.1) has at least one nontrivial solution provided that either $a<a_{0}$ or $a_{0}<\lambda_{k}<a$ for some $k>m$ and (4.14)-(4.15) hold with $m=k$.

(c) If $a_{0}=\lambda_{m}$ is an eigenvalue but (4.14)-(4.15) and $\left(\mathrm{q}_{4}^{-}\right)$hold in addition, then (4.1) has at least one nontrivial solution provided that either $a_{0}<a$ or $a<\lambda_{k}<a_{0}$ for some $k<m$ and (4.14) holds with $m=k$.

Indeed, the condition $\left(\mathrm{q}_{4}^{+}\right)$(resp. $\left.\left(\mathrm{q}_{4}^{+}\right)\right)$is used to assure that the local linking condition in Propositions 2.3 of [2] holds with $X^{-}=H_{\theta}^{0} \oplus H_{\theta}^{-}$and $X^{+}=H_{\theta}^{+}$ (resp. $X^{-}=H_{\theta}^{-}$and $X^{+}=H_{\theta}^{0} \oplus H_{\theta}^{+}$) because

$$
J(u)=\frac{1}{2}(B(\theta) u, u)_{H}-\int_{\Omega} Q_{0}(x, u(x)) d x \quad \text { for all } u \in H .
$$

They imply $C_{\mu_{\theta}+\nu_{\theta}}(J, \theta) \neq 0$ and $C_{\mu_{\theta}}(J, \theta) \neq 0$, respectively.

There exists a further possible improvement, that is, the limit $a_{0}=\lim _{t \rightarrow 0} \frac{p(x, t)}{t}$ in $\left(\mathrm{p}^{*}\right)$ is not required to be constant. For example, for Theorem 4.7(a), we may assume that $a_{0}(x)=\lim _{t \rightarrow 0} p(x, t) / t$ exists for almost every $x \in \Omega$. Then the second condition in $\left(\mathrm{p}^{*}\right)$ and $\left(\mathrm{q}_{3}^{*}\right)$ imply that $a_{0}(x)=a+q_{t}(x, 0)$ for almost every $x \in \Omega$. Suppose that 1 is not an eigenvalue of the equation $-\triangle u=\lambda a_{0} u$ in $\Omega$ with 0 boundary conditions. Then $\theta$ is a nondegenerate critical point of $J$ with finite Morse index $\mu_{\theta}$, and hence $C_{k}(J, \theta)=\delta_{k \mu_{\theta}} \mathbb{K}$. If $\theta$ is a unique critical point of $J$ then $C_{k}(J, \infty)=\delta_{k \mu_{\theta}} \mathbb{K}$ by Proposition 3.6 of [2]. Proposition 4.6 shall lead to a contradiction under the suitable condition on $a$. The corresponding generalizations of Theorem 4.7 (b)-(c) can be obtained similarly.

Proof of Proposition 4.6. Step 1. Carefully checking the proof of Lemma 4.2 in [2] one easily sees that (4.10) and (4.12) imply the corresponding result: For sufficiently large $R>0$ and $b \ll 0$ the pair

$$
\left(B_{H_{\infty}^{0}}(\theta, R+1) \oplus H_{\infty}^{ \pm}, J^{b} \cap\left(B_{H_{\infty}^{0}}(\theta, R+1) \oplus H_{\infty}^{ \pm}\right)\right)
$$

is homotopy to the pair

$$
\left(B_{H_{\infty}^{0}}(\theta, R+1) \oplus \bar{B}_{H_{\infty}^{-}}(\theta, 1), B_{H_{\infty}^{0}}(\theta, R+1) \oplus \partial \bar{B}_{H_{\infty}^{-}}(\theta, 1)\right) .
$$

The homotopy equivalence leaves the $H_{\infty}^{0}$-component fixed. In particular, the pair $\left(H, J^{b}\right)$ is homotopy to the pair $\left(\bar{B}_{H_{\infty}^{-}}(\theta, 1), \partial \bar{B}_{H_{\infty}^{-}}(\theta, 1)\right)$ provided that $\nu_{\infty}=0$ and $\mu_{\infty}<\infty$.

The final claim immediately leads to (a) and (b).

Step 2. We begin to prove (c). In this case obverse that

$$
\left(\left.B(\infty)\right|_{H_{\infty}^{ \pm}}\right)^{-1}\left(\sum_{\lambda_{j} \neq \lambda_{m}} x_{j} \varphi_{j}\right)=\sum_{\lambda_{j} \neq \lambda_{m}} \frac{\lambda_{j}}{\lambda_{j}-\lambda_{m}} x_{j} \varphi_{j} .
$$


For $X=H$, by the definitions of $C_{1}^{\infty}$ and $C_{2}^{\infty}$ above (1.2) we have

$$
C_{1}^{\infty}=\left\|\left(\left.B(\infty)\right|_{H_{\infty}^{ \pm}}\right)^{-1}\right\|_{L\left(H_{\infty}^{ \pm}, H_{\infty}^{ \pm}\right)} \quad \text { and } \quad C_{2}^{\infty}=\left\|I-P_{\infty}^{0}\right\|_{L\left(H, H_{\infty}^{ \pm}\right)} .
$$

From these ones easily derive

Lemma 4.8. $C_{2}^{\infty}=1$ (because $I-P_{\infty}^{0}=P_{\infty}^{ \pm} \neq I$ ). If $a=\lambda_{1}$ then

$$
C_{1}^{\infty}=\left\|\left(\left.B(\infty)\right|_{H_{\infty}^{ \pm}}\right)^{-1}\right\|_{\mathcal{L}\left(H_{\infty}^{ \pm}\right)}=\frac{\lambda_{2}}{\lambda_{2}-\lambda_{1}},
$$

and if $a=\lambda_{m}$ with $m \geq 2$ then

$$
C_{1}^{\infty}=\left\|\left(\left.B(\infty)\right|_{H_{\infty}^{ \pm}}\right)^{-1}\right\|_{\mathcal{L}\left(H_{\infty}^{ \pm}\right)}=\max \left\{\frac{\lambda_{m^{-}-1}}{\lambda_{m}-\lambda_{m^{-}-1}}, \frac{\lambda_{m^{+}+1}}{\lambda_{m^{+}+1}-\lambda_{2}}\right\} .
$$

We wish to use Corollary 1.6. It suffices to check the conditions (c) $-(\mathrm{d})$ therein. Since $Q(\infty) v=-a K v, B(u) v-Q(\infty) v=v-K\left(\bar{q}_{t}(x) v\right)$ and hence

$$
\begin{aligned}
(B(u) v-Q(\infty) v, v)_{H} & =(v, v)_{H}-\int_{\Omega} q_{t}(x, u(x))(v(x))^{2} d x \\
& \geq(v, v)_{H}-\left(\int_{\Omega} \ell(x) d x\right) \cdot\|h\|_{L^{\infty}} \cdot\|u\|_{C^{0}} \cdot\|v\|_{C^{0}} \\
& \geq\left(1-c(\Omega)^{2}\|\ell\|_{1} \cdot\|h\|_{L^{\infty}}\right)\|u\|_{H}^{2} .
\end{aligned}
$$

This shows that the conditions (c) of Corollary 1.6 holds under (4.15).

By (4.12), $\|\nabla J(z)\|_{H}=o\left(\|z\|_{H}\right)$ as $z \in H_{\infty}^{0}$ and $\|u\|_{H} \rightarrow \infty$. Hence

$$
M(A)=M(\nabla J)=\lim _{R \rightarrow \infty} \sup \left\{\left\|\left(I-P_{\infty}^{0}\right) \nabla J(z)\right\|_{H}: z \in H_{\infty}^{0},\|z\|_{H} \geq R\right\}=0 .
$$

By Lemma 4.8 and (4.14) we may take a small $\varepsilon>0$ such that

$$
c(\Omega)^{2}\|\ell\|_{s}\left(\varepsilon+\hbar|\Omega|^{\iota(s)}\right)<1 / C_{1}^{\infty} .
$$

For this $\varepsilon>0$ and a given numbers $\rho>0$, by Claim 4.4 there exist $R_{0}>0$ such that

$$
\sup _{\tau \in[0,1]}\|h(z+u)-\hbar\|_{s_{1} s /\left(s-s_{1}\right)}+\hbar|\Omega|^{\iota(s)}<\varepsilon+\hbar|\Omega|^{\iota(s)}
$$

for any $u \in \bar{B}_{H_{\infty}^{ \pm}}(\theta, \rho)$ and $z \in H_{\infty}^{0}$ with $\|z\|_{H} \geq R_{0}$. This and (4.11) lead to

$$
\begin{aligned}
\left\|\left.\left(I-P_{\infty}^{0}\right)[B(z+u)-B(\infty)]\right|_{H_{\infty}^{ \pm}}\right\|_{L\left(H_{\infty}^{ \pm}\right)} \leq\|B(z+u)-B(\infty)\|_{L(H)} \\
\quad=\left\|g^{\prime \prime}(z+u)\right\|_{L(H)} \leq c(\Omega)^{2}\|\ell\|_{s}\left(\varepsilon+\hbar|\Omega|^{\left(s-s_{1}\right) / s_{1} s}\right)<\frac{1}{\kappa C_{1}^{\infty}}
\end{aligned}
$$

for any $u \in \bar{B}_{H_{\infty}^{ \pm}}(\theta, \rho)$ and $z \in H_{\infty}^{0}$ with $\|z\|_{H} \geq R_{0}$. Summarizing these we obtain

Lemma 4.9. For $a=\lambda_{m}$, if either $\hbar=0$ or $\hbar>0$ and (4.14)-(4.15) are satisfied, then taking $\rho_{\nabla J}$ as any positive number $\rho$ there exist $R_{1}>0$ such that the conditions of Corollary 1.6 is satisfied. 
Under the assumptions of Proposition 4.2 and Lemma 4.9, by Corollary 1.6 there exist a positive number $R$, a $C^{1}$ map $h^{\infty}: B_{H_{\infty}^{0}}(\infty, R) \rightarrow \bar{B}_{H_{\infty}^{ \pm}}\left(\theta, \rho_{\nabla J}\right)$ (satisfying $\left(I-P_{\infty}^{0}\right) A\left(z+h^{\infty}(z)\right)=0$ for all $\left.z \in \bar{B}_{H_{\infty}^{0}}(\infty, R)\right)$, and a homeomorphism $\Phi: B_{H_{\infty}^{0}}(\infty, R) \oplus H_{\infty}^{ \pm} \rightarrow B_{H_{\infty}^{0}}(\infty, R) \oplus H_{\infty}^{ \pm}$such that

$$
J \circ \Phi\left(z+u^{+}+u^{-}\right)=\left\|u^{+}\right\|^{2}-\left\|u^{-}\right\|^{2}+J\left(z+h^{\infty}(z)\right)
$$

for all $\left(z, u^{+}+u^{-}\right) \in B_{H_{\infty}^{0}}(\infty, R) \times H_{\infty}^{ \pm}$. Using this we may repeat the arguments on pages 432-433 of [2] to derive that Lemma 4.3 of [2] holds for $J$ : There exist a sufficiently large $R>0, b \ll 0$ and a continuous map $\gamma: B_{H_{\infty}^{0}}(\infty, R) \rightarrow[0,1]$ with $\gamma(C)>0$ for $C:=B_{H_{\infty}^{0}}(\theta, R+1) \cap B_{H_{\infty}^{0}}(\infty, R)$ such that the pair

$$
\left(B_{H_{\infty}^{0}}(\infty, R) \times H_{\infty}^{ \pm}, J^{b} \cap\left(B_{H_{\infty}^{0}}(\infty, R) \times H_{\infty}^{ \pm}\right)\right)
$$

is homotopy equivalent to the pair $\left(B_{H_{\infty}^{0}}(\infty, R) \times H_{\infty}^{-}, \Gamma\right)$, where

$$
\begin{aligned}
& \Gamma=\left\{(z, u) \in B_{H_{\infty}^{0}}(\infty, R) \times H_{\infty}^{-}:\|u\| \geq \gamma(z)\right\} \quad \text { and } \\
& \gamma(z)= \begin{cases}0 & \text { if } J\left(z+h^{\infty}(z)\right) \leq a, \\
1 & \text { if } J\left(z+h^{\infty}(z)\right) \geq a+1, \\
J\left(z+h^{\infty}(z)\right)-b & \text { elsewhere. }\end{cases}
\end{aligned}
$$

Moreover, the homotopy equivalence leaves the $H_{\infty}^{0}$-component fixed.

Combing this with Step 1 and repeating the proof of Theorem 3.9 in [2] we get the claim in Proposition 4.6(c), i.e. $C_{k}(J, \infty ; \mathbb{K}) \cong H_{k}\left(H, J^{b} ; \mathbb{K}\right)=0$ for all $k \notin\left[m^{-}-1, m^{+}\right]$because $\left[\mu_{\infty}, \mu_{\infty}+\nu_{\infty}\right]=\left[m^{-}-1, m^{+}\right]$by the list above Proposition 4.5.

Proof of Proposition 4.2. (a) Since the functional $H \ni u \mapsto(B(\infty) u, u)_{H}$ is smooth, we only need to prove that the functional $g$ in (4.8) is $C^{1}$. Clearly, it suffices to prove that $g$ is $C^{1}$ under the assumption: there exist $E \in L^{1}(\Omega)$ and $r \geq 1$ such that

$$
|q(x, t)| \leq E(x)+|t|^{r} \quad \text { for a.e. } x \in \Omega \text {, for all } t \in \mathbb{R} .
$$

Obverse that $Q$ is also a Carthéodory function and that

$$
\begin{aligned}
|Q(x, u(x)+v(x))-Q(x, u(x))| & =\left|\int_{u(x)}^{u(x)+v(x)} q(x, \tau) d \tau\right| \\
& =\sup _{\tau \in[0,1]}|q(x, u(x)+\tau v(x))| \cdot|u(x)-v(x)| \\
& \leq\left(E(x)+\sup _{\tau \in[0,1]}|u(x)+\tau v(x)|^{r}\right) \cdot|u(x)-v(x)| \\
& \leq\left(E(x)+\left(\|u\|_{C^{0}}+\|v(x)\|_{C^{0}}\right)^{r}\right) \cdot\|u-v\|_{C^{0}}
\end{aligned}
$$

for any $u, v \in H$. So $g$ (and hence $J$ ) is continuous because

$$
|g(u+v)-g(u)| \leq\left(\|E\|_{L^{1}}+|\Omega| \cdot\left(\|u\|_{C^{0}}+\|v(x)\|_{C^{0}}\right)^{r}\right) \cdot\|u-v\|_{C^{0}} .
$$


In order to prove that $g$ is $C^{1}$, by the standard result in functional analysis we only need to prove that $g$ has a bounded linear Gâteaux derivative $D g(u)$ at every point $u \in H$ and that $H \ni u \mapsto D g(u) \in H^{*}$ is continuous.

For $u, v \in H_{0}^{1}(\Omega), \tau \in(-1,1) \backslash\{0\}$ and almost every $x \in \Omega$, as above we get

$$
\begin{aligned}
\mid \frac{Q(x, u(x)+\tau v(x))-Q(x}{\tau}, & u(x))|=| \frac{1}{\tau} \int_{u(x)}^{u(x)+\tau v(x)} q(x, \tau) d \tau \mid \\
& \leq \sup _{0<\theta<1}|q(x, u(x)+\theta \tau v(x)) v(x)| \cdot|v(x)| \\
& \leq\left(E(x)+\left(\|u\|_{C^{0}}+\|v(x)\|_{C^{0}}\right)^{r}\right) \cdot\|v\|_{C^{0}}
\end{aligned}
$$

by (4.16). From this and the Lebesgue dominated convergence theorem we derive

$$
D g(u)[v]=\left.\frac{d}{d \tau}\right|_{\tau=0} g(u+\tau v)=-\int_{\Omega} q(x, u(x)) \cdot v(x) d x
$$

That is, $g$ is Gâteaux differentiable. Clearly, $D g(u) \in H^{*}$. Since $H_{0}^{1}(\Omega) \hookrightarrow$ $C(\bar{\Omega})$ and $|q(x, u(x))| \leq E(x)+\|u\|_{C^{0}}^{r}$ by (4.16), as above we deduce that $H \times$ $H \ni(u, v) \mapsto D g(u)[v]$ is continuous. That is, $g$ is continuously directional differentiable.

Moreover, for $u_{1}, u_{2}, v \in H_{0}^{1}(\Omega),(4.5)$ leads to

$\left|\int_{\Omega}\left[q\left(x, u_{2}(x)\right)-q\left(x, u_{1}(x)\right)\right] \cdot v(x) d x\right| \leq\|v\|_{C^{0}} \int_{\Omega}\left|q\left(x, u_{2}(x)\right)-q\left(x, u_{1}(x)\right)\right| d x$ and hence

$$
\begin{aligned}
\left\|D g\left(u_{1}\right)-D g\left(u_{2}\right)\right\|_{H^{*}} & =\sup _{\|v\|_{H} \leq 1}\left|D g\left(u_{1}\right) v-D g\left(u_{2}\right) v\right| \\
& \leq c(\Omega) \int_{\Omega}\left|q\left(x, u_{2}(x)\right)-q\left(x, u_{1}(x)\right)\right| d x
\end{aligned}
$$

where $c(\Omega)$ is as in (4.2). Let $f(x)$ denote the sign function of $q\left(x, u_{2}(x)\right)-$ $q\left(x, u_{1}(x)\right)$. It is measurable, and

$$
\int_{\Omega}\left|q\left(x, u_{2}(x)\right)-q\left(x, u_{1}(x)\right)\right| d x=\int_{\Omega}\left[f(x) q\left(x, u_{2}(x)\right)-f(x) q\left(x, u_{1}(x)\right)\right] d x .
$$

Obverse that $\Omega \times \mathbb{R} \ni(x, t) \mapsto f(x) q(x, t)$ is a also a Carthéodory function and that $|f(x) q(x, t)| \leq|q(x, t)| \leq E(x)+|t|^{r}$ for almost every $x \in \Omega$ and all $t \in \mathbb{R}$. By the standard properties of the Nemytski operator (cf. [8, Proposition 3.2.24]), the map $L^{r}(\Omega) \ni u \mapsto \mathfrak{q}(u) \in L^{1}(\Omega)$, where $\mathfrak{q}(u)(x)=f(x) q(x, u(x))$, is continuous. From the continuity of the inclusion $H \hookrightarrow L^{r}(\Omega)$ it follows that the functional $D g$ is continuous. Hence $g$ (and therefore $J$ ) is $C^{1}$.

Finally, since $|q(x, t)| \leq E(x)+c_{1}|t|^{r} \forall(x, t) \in \Omega \times \mathbb{R}$ by $\left(q_{1}^{*}\right)$, we derive

$$
|Q(x, t)|=\left|\int_{0}^{t} q(x, \tau) d x\right| \leq|t| E(x)+c_{1}|t|^{r+1} \quad \text { for all }(x, t) \in \Omega \times \mathbb{R} .
$$


Hence for any $u \in H$ we have

$$
\begin{aligned}
|g(u)| & \leq \int_{\Omega}|Q(x, u(x))| d x \leq \int_{\Omega}\left(E(x)|u(x)|+c_{1}|u(x)|^{r+1}\right) d x \\
& \leq\|u\|_{C^{0}} \int_{\Omega} E(x) d x+c_{1}|\Omega| \cdot\|u\|_{C^{0}}^{r+1} \\
& \leq c(\Omega)\|u\|_{H} \int_{\Omega} E(x) d x+c_{1}(c(\Omega))^{r+1}|\Omega| \cdot\|u\|_{H}^{r+1},
\end{aligned}
$$

where $c(\Omega)$ is as in (4.2). (4.10) follows. The expression of $\nabla J$ is clear.

(b) For the integer $s^{\prime}$ satisfying the equality $1 / s+1 / s^{\prime}=1$, by (4.5),

$$
|q(x, t)| \leq\left(\frac{1}{s}(\ell(x))^{s}+\frac{1}{s^{\prime}}|t|^{s^{\prime}}\right) \cdot\|h\|_{L^{\infty}} \quad \text { for a.e. } x \in \Omega \text {, for all } t \in \mathbb{R} .
$$

So it follows from (a) that $g$ and hence $J$ is $C^{1}$.

Let us prove that $\nabla J$ is $C^{1}$. For $u, v, w \in H$ and $\tau \in(-1,1) \backslash\{0\}$, we have

$$
\begin{aligned}
& \left|\left(\frac{K(\bar{q}(u+\tau v))-K(\bar{q}(u))}{\tau}, w\right)_{H}-\left(K\left(\bar{q}_{t}(u) v\right), w\right)_{H}\right| \\
& \quad=\left|\int_{\Omega}\left[\frac{q(x, u(x)+\tau v(x))-q(x, u(x))}{\tau}-q_{t}(x, u(x)) v(x)\right] \cdot w(x) d x\right| \\
& \quad \leq \int_{\Omega}\left|\frac{q(x, u(x)+\tau v(x))-q(x, u(x))}{\tau}-q_{t}(x, u(x)) v(x)\right| \cdot|w(x)| d x
\end{aligned}
$$

and thus

$$
\begin{aligned}
& \left\|\frac{K(\bar{q}(u+\tau v))-K(\bar{q}(u))}{\tau}-K\left(\bar{q}_{t}(u) v\right)\right\|_{H} \\
& \quad \leq c(\Omega) \int_{\Omega}\left|\frac{q(x, u(x)+\tau v(x))-q(x, u(x))}{\tau}-q_{t}(x, u(x)) v(x)\right| d x .
\end{aligned}
$$

Note that for almost every $x \in \Omega$,

$$
\left|\frac{q(x, u(x)+\tau v(x))-q(x, u(x))}{\tau}-q_{t}(x, u(x)) v(x)\right| \leq 2 \ell(x)\|h\|_{L^{\infty}} \cdot|v(x)|
$$

by $\left(\mathrm{q}_{3}^{*}\right)$. From the Lebesgue dominate convergence theorem we derive

$$
\begin{aligned}
\lim _{\tau \rightarrow 0} \| & \left\|\frac{K(\bar{q}(u+\tau v))-K(\bar{q}(u))}{\tau}-K\left(\bar{q}_{t}(u) v\right)\right\|_{H} \\
& \leq \lim _{\tau \rightarrow 0} \int_{\Omega}\left|\left[\frac{q(x, u(x)+\tau v(x))-q(x, u(x))}{\tau}-q_{t}(x, u(x)) v(x)\right]\right| d x=0 .
\end{aligned}
$$

Hence $\nabla g$ is Gâteaux differentiable, and $\nabla J$ has the Gâteaux derivative $B(u)$ at any $u \in H$. Moreover, obverse that

$$
\left(B\left(u_{1}\right) v-B\left(u_{2}\right) v, w\right)_{H}=\int_{\Omega}\left[q_{t}\left(x, u_{2}(x)\right)-q_{t}\left(x, u_{1}(x)\right)\right] v(x) w(x) d x
$$


and hence

$$
\begin{aligned}
\left\|B\left(u_{1}\right) v-B\left(u_{2}\right) v\right\|_{H} & \leq c(\Omega)\|v\|_{C^{0}} \int_{\Omega}\left|q_{t}\left(x, u_{2}(x)\right)-q_{t}\left(x, u_{1}(x)\right)\right| d x \\
& \leq c(\Omega)^{2}\|v\|_{H} \int_{\Omega}\left|q_{t}\left(x, u_{2}(x)\right)-q_{t}\left(x, u_{1}(x)\right)\right| d x .
\end{aligned}
$$

We obtain

$$
\left\|B\left(u_{1}\right)-B\left(u_{2}\right)\right\|_{\mathcal{L}(H)} \leq c(\Omega)^{2} \int_{\Omega}\left|q_{t}\left(x, u_{2}(x)\right)-q_{t}\left(x, u_{1}(x)\right)\right| d x .
$$

Since $t \mapsto q_{t}(x, t)$ is continuous, $q_{t}\left(x, u_{2}(x)\right) \rightarrow q_{t}\left(x, u_{1}(x)\right)$ as $\left\|u_{2}-u_{1}\right\|_{C^{0}} \rightarrow 0$. It follows from $\left(q_{3}^{*}\right)$ that the map $H \ni u \mapsto B(u) \in \mathcal{L}(H)$ is continuous. This implies that $\nabla J$ has the Fréchlet derivative $B(u)$ at $u \in H$, and therefore that $J$ is $C^{2}$.

In order to prove (4.11), note that for $z \in H_{\infty}^{0}$ and $u_{1} \in H_{\infty}^{ \pm}, u_{2} \in H$ we have

$$
\begin{aligned}
\left\|g^{\prime \prime}\left(z+u_{1}\right) u_{2}\right\|_{H}=\sup _{\|w\|_{H} \leq 1}\left|\left(g^{\prime \prime}\left(z+u_{1}\right) u_{2}, w\right)_{H}\right| \\
=\sup _{\|w\|_{H} \leq 1} \mid\left(K\left(\bar{q}_{t}\left(z+u_{1}\right) u_{2}, w\right)_{H} \mid\right. \\
\leq \sup _{\|w\|_{H} \leq 1} \int_{\Omega}\left|q_{t}\left(x, z(x)+u_{1}(x)\right) u_{2}(x) w(x)\right| d x \\
\leq \sup _{\|w\|_{H} \leq 1} \int_{\Omega} \ell(x) h\left(z(x)+u_{1}(x)\right)\left|u_{2}(x) w(x)\right| d x \\
\leq\left\|u_{2}\right\|_{C^{0}}\left(\sup _{\|w\|_{H} \leq 1}\|w\|_{C^{0}}\right) \int_{\Omega} \ell(x) h\left(z(x)+u_{1}(x)\right) d x \\
\leq\|\ell\|_{s}\left(\int_{\Omega}\left|h\left(z(x)+u_{1}(x)\right)\right|^{s_{1} s /\left(s-s_{1}\right)} d x\right)^{\left(s-s_{1}\right) /\left(s_{1} s\right)} \\
\quad \cdot\left\|u_{2}\right\|_{C^{0}}\left(\sup _{\|w\|_{H} \leq 1}\|w\|_{C^{0}}\right) \\
\leq\|\ell\|_{s}\left\|h\left(z+u_{1}\right)\right\|_{s_{1} s /\left(s-s_{1}\right)} \cdot\left\|u_{2}\right\|_{C^{0}}\left(\sup _{\|w\|_{H} \leq 1}\|w\|_{C^{0}}\right) \\
\leq c(\Omega)^{2}\|\ell\|_{s}\left[\left\|h\left(z+u_{1}\right)-\hbar\right\|_{s_{1} s /\left(s-s_{1}\right)} \hbar|\Omega|^{\left(s-s_{1}\right) /\left(s_{1} s\right)}\right] \cdot\left\|u_{2}\right\|_{H}
\end{aligned}
$$

where $c(\Omega)$ is as in (4.2). (4.11) follows from this.

(c) Since $\nabla J(u)-B(\infty) u=\nabla g(u)$, we may derive (4.12) as follows:

$$
\begin{aligned}
\|\nabla g(u)\|_{H} & =\sup _{\|v\|_{H} \leq 1}\left|(\nabla g(u), v)_{H}\right| \leq \sup _{\|v\|_{H} \leq 1} \int_{\Omega}|q(x, u(x))| \cdot|v(x)| d x \\
& \leq\left(\sup _{\|v\|_{H} \leq 1}\|v\|_{C^{0}}\right) \cdot \int_{\Omega}|q(x, u(x))| d x \\
& \leq c(\Omega) \int_{\Omega} E(x) d x+c_{1} c(\Omega) \int_{\Omega}|u(x)|^{r} d x
\end{aligned}
$$




$$
\leq c(\Omega) \int_{\Omega} E(x) d x+c_{1} c(\Omega)^{r+1}|\Omega| \cdot\|u\|_{H}^{r} .
$$

(d) By (4.7), $B(\infty)=I-a K$. Let $H_{\infty}^{0}:=\operatorname{Ker}(B(\infty))$. Note that the positive (resp. negative) definite subspace of $B(\infty), H_{\infty}^{+}$(resp. $H_{\infty}^{-}$), is spanned by the eigenfunctions of $-\triangle$ which correspond to the eigenvalues less than (resp. greater than) $a$. Since $H=H_{\infty}^{0} \oplus H_{\infty}^{+} \oplus H_{\infty}^{-}$we may write $u \in H$ as $u=u^{0}+u^{+}+u^{-}$. Hence $(B(\infty) u, u)_{H}=\left(B(\infty) u^{+}, u^{+}\right)_{H}+\left(B(\infty) u^{-}, u^{-}\right)_{H}$. It follows that

$$
\|u\|_{*}=\left(\left\|u^{0}\right\|_{L^{2}}^{2}+\left(B(\infty) u^{+}, u^{+}\right)_{H}-\left(B(\infty) u^{-}, u^{-}\right)_{H}\right)^{1 / 2}
$$

defines an equivalent norm on $H$. Obverse that

$$
\|u\|_{*}=\left(\int_{\Omega}\left\|u^{0}\right\|^{2} d x+\int_{\Omega}\left[\left|\nabla u^{+}\right|^{2}-a\left|u^{+}\right|^{2}\right] d x-\int_{\Omega}\left[\left|\nabla u^{-}\right|^{2}-a\left|u^{-}\right|^{2}\right] d x\right)^{1 / 2} .
$$

Let $\left(u_{n}\right)$ be a Palais-Smale sequence for $J$ in $H$. That is, $J^{\prime}\left(u_{n}\right) \rightarrow 0$ and $\left|J\left(u_{n}\right)\right| \leq M$ for some $M>0$ and all $n \in \mathbb{N}$. As in the proof of Lemma 5.5 in [2] we have

$$
\left(\left\|u_{n}^{ \pm}\right\|_{*}\right)^{2} \leq\left|\int_{\Omega} q\left(x, u_{n}\right) u_{n}^{ \pm} d x\right|+\left\|u_{n}^{ \pm}\right\|_{*}
$$

for $n$ large. From $\left(\mathrm{q}_{1}^{*}\right)$ we derive

$$
\left|\int_{\Omega} q\left(x, u_{n}\right) u_{n}^{ \pm} d x\right| \leq \int_{\Omega}\left|q\left(x, u_{n}\right)\right| \cdot\left|u_{n}^{ \pm}\right| d x \leq\left\|u_{n}^{ \pm}\right\|_{C^{0}} \int_{\Omega}\left(E(x)+c_{1}\left|u_{n}(x)\right|^{r}\right) d x .
$$

Since $H \hookrightarrow C(\bar{\Omega})$ and the norms $\|\cdot\|_{H}$ and $\|\cdot\|_{*}$ on $H$ are equivalent, there exists a constant only depending on $\Omega, C_{\Omega}>0$, such that $\|u\|_{C^{0}} \leq C_{\Omega}\|u\|_{*}$ for all $u \in H$. So

$$
\left|\int_{\Omega} q\left(x, u_{n}\right) u_{n}^{ \pm} d x\right| \leq C_{\Omega}\left\|u_{n}^{ \pm}\right\|_{*} \cdot\left(\|E\|_{L^{1}}+c_{1} C_{\Omega}^{r}|\Omega| \cdot\left\|u_{n}\right\|_{*}^{r}\right) .
$$

These lead to $\left\|u_{n}^{ \pm}\right\|_{*} \leq 1+C_{\Omega} \cdot\|E\|_{L^{1}}+c_{1} C_{\Omega}^{r+1}|\Omega| \cdot\left\|u_{n}\right\|_{*}^{r}$. By ( $\left(\mathrm{q}_{2}^{*}\right)$ we may assume

$$
\frac{1}{2} q(x, t) t-Q(x, t) \geq c_{2}|t|^{\alpha}-G(x) \quad \text { for all }(x, t) \in \Omega \times \mathbb{R} .
$$

(Another case can be proved in the same way). As in [2] we have

$$
\begin{aligned}
M+\left\|u_{n}\right\|_{*} & \geq\left|J\left(u_{n}\right)-\frac{1}{2} J^{\prime}\left(u_{n}\right) u_{n}\right| \\
& =\left|\int_{\Omega}\left(\frac{1}{2} q\left(x, u_{n}(x)\right) u_{n}(x)-Q\left(x, u_{n}(x)\right)\right) d x\right| \\
& \geq\left|\int_{\Omega}\left(\frac{1}{2} q\left(x, u_{n}(x)\right) u_{n}(x)-Q\left(x, u_{n}(x)+G(x)\right)\right) d x\right|-\|G\|_{L^{1}} \\
& \geq c_{2} \int_{\omega}\left|u_{n}(x)\right|^{\alpha} d x-\|G\|_{L^{1}} .
\end{aligned}
$$

Then repeating the remainder arguments in the proof of Lemma 5.5 in [2] give the desired conclusion. 


\section{REFERENCES}

[1] P. Bartolo, V. Benci and D. Fortunato, Abstract critical point theorems and applications to some nonlinear problems with "strong" resonance at infinity, Nonlinear Anal. 7 (1983), 981-1012.

[2] T. BARTSCH AND S.-J. LI, Critical point theory for asymptotically quadratic functionals and applications to problems with resonance, Nonlinear Anal. 28 (1997), no. 3, 419-441.

[3] H. Brezis, Functional Analysis, Sobolev Spaces and Partial Differential Equations, Springer 2011.

[4] K.C. Chang, Infinite Dimensional Morse Theory and Multiple Solution Problem. Birkhäuser, 1993.

[5] S.W. Chen And S.-J. Li, Splitting lemma in the infinity and a strong resonant problem with periodic nonlinearity, Nonlinear Anal. 65 (2006), 567-582.

[6] __ Splitting lemma at infinity and a strongly resonant problem with periodic nonlinearity, Calc. Var. 27 (2006), no. 1, 105-123.

[7] J.B. Conway, A Course in Functional Analysis, Springer, New York, 1990.

[8] P. Drabek and J. Milota, Methods of Nonlinear Analysis. Applications to Differential Equations, Birkhüser Advanced Texts: Basler Lehrbücher. Birkhüser Verlag, Basel, 2007.

[9] D.M. Duc, T.V. Hung And N.T. KhaI, Morse-Palais lemma for nonsmooth functionals on normed spaces, Proc. Amer. Math. Soc. 135 (2007), no. 3., 921-927.

[10] N. Hirano, S.-J. Li And Z.Q. WANG, Morse theory without (PS) condition at isolated values and strong resonance problem, Calc. Var. Partial Differential Equations, 10 (2000), 223-247.

[11] M. JiAng, A generalization of Morse lemma and its applications, Nonlinear Anal. 36 (1999), 943-960.

[12] S.-J. LI, A new Morse theory and strong resonance problems, Topol. Methods Nonlinear Anal. 21 (2003), 81-100.

[13] G. Lu, The splitting lemmas for nonsmooth functionals on Hilbert spaces, arXiv:1102. $2062 \mathrm{v} 1$.

[14] _ The splitting lemmas for nonsmooth functionals on Hilbert spaces I, Discrete Contin. Dyn. Syst. 33 (2013), no. 7, 2939-2990.

[15] _ Some critical point theorems and applications, arXiv:1102.2136v1.

[16] J. Mawhin and M. Willem, Critical Point Theory and Hamiltonian Systems, Applied Mathematical Sciences Vol. 74., Springer-Verlag, 1989.

[17] I.V. Skrypnik, Nonlinear Elliptic Equations of a Higher Order, Naukova Dumka, Kiev (1973). (in Russian)

[18] S.A. Vakhrameev, Critical point theory for smooth functions on Hilbert manifolds with singularities and its application to some optimal control problems, J. Sov. Math. 67 (1993), No. 1, 2713-2811.

GuANGCun Lu

School of Mathematical Sciences

Beijing Normal University

Laboratory of Mathematics and Complex Systems

Ministry of Education

Beijing 100875, The P.R. CHINA

E-mail address: gclu@bnu.edu.cn

TMNA : Volume $44-2014-\mathrm{N}^{\mathrm{O}} 2$ 